\title{
Untanglings: A Novel Approach to Analyzing Concurrent Systems
}

\author{
Artem Polyvyanyy ${ }^{1}$, Marcello La Rosa ${ }^{1,2}$, Chun Ouyang ${ }^{1}$, Arthur H.M. ter Hofstede ${ }^{1,3}$ \\ ${ }^{1}$ Queensland University of Technology, Brisbane, Australia \\ ${ }^{2}$ NICTA Queensland lab, Brisbane, Australia \\ ${ }^{3}$ Eindhoven University of Technology, Eindhoven, The Netherlands
}

\begin{abstract}
Substantial research efforts have been expended to deal with the complexity of concurrent systems that is inherent to their analysis, e.g., works that tackle the well-known state space explosion problem. Approaches differ in the classes of properties that they are able to suitably check and this is largely a result of the way they balance the trade-off between analysis time and space employed to describe a concurrent system. One interesting class of properties is concerned with behavioral characteristics. These properties are conveniently expressed in terms of computations, or runs, in concurrent systems. This article introduces the theory of untanglings that exploits a particular representation of a collection of runs in a concurrent system. It is shown that a representative untangling of a bounded concurrent system can be constructed that captures all and only the behavior of the system. Representative untanglings strike a unique balance between time and space, yet provide a single model for the convenient extraction of various behavioral properties. Performance measurements in terms of construction time and size of representative untanglings with respect to the original specifications of concurrent systems, conducted on a collection of models from practice, confirm the scalability of the approach. Finally, this article demonstrates practical benefits of using representative untanglings when checking various behavioral properties of concurrent systems.
\end{abstract}

Keywords: Concurrency; Concurrent systems; Analysis; Untanglings; Representative untanglings

\section{Introduction}

Concurrent systems are systems in which multiple computations, or runs, are executed simultaneously by independent processing units, which usually interact with each other, in order to achieve a common goal. Naturally, concurrent systems can provide a better 'throughput', i.e., produce more computed output relative to the number and size of given tasks over a certain period of time, than systems in which all operations are performed in a centralized manner by a single processing unit. The design of a concurrent system, thus, deals with finding a suitable coordination of operations that encourages efficiency of individual computations.

Concurrent systems are complex to analyze. The complexity is primarily due to the amount of uncertainty

Correspondence and offprint requests to: Artem Polyvyanyy, Queensland University of Technology, GPO Box 2434, Brisbane, QLD 4001, Australia. e-mail: artem.polyvyanyy@qut.edu.au 
about the order in which atomic operations can be executed by different processing units of a system. When studying this fact in detail, one can immediately identify the following three reasons that are inherent to the complexity of concurrent systems:

- Firstly, the number of runs described in a concurrent system is exponential in the number of choices that one can make when carving a way through the system into a desired computation.

- Secondly, the number of all possible interleavings of operations described in a concurrent system grows at a factorial rate with respect to the number of simultaneously enabled operations - a phenomenon which leads to the well-known state space explosion problem.

- Thirdly, a concurrent system can encode an infinite number of runs in the case of loops.

Consequently, approaches to analyzing concurrent systems constitute one of the most complex classes of practical problems in computer science. Most of the techniques for verification of behavioral characteristics of concurrent systems, i.e., properties over potential runs of concurrent systems, are doomed to explore immense portions of operation interleavings that are encoded in models of concurrent systems. Besides, the complexity of these explorations depends on the particular formalism that is used to describe systems.

A number of formalisms for modeling and analyzing concurrent systems have been developed and systematized [SNW96, LSV98]. Some of them can be used through the entire development cycle while others target some particular phase, e.g., analysis. One of the first formalisms for describing concurrent systems, and by now one of the most studied, was proposed in Carl Adam Petri's seminal work on Petri nets [Pet77]. A concurrent system captured in the Petri net notation, or a net system, is often reminiscent of a mass of highly interwoven models of individual runs - a tangle of computations. Every time a Petri net gets instantiated it 'works' its way through a maze of pre-designed options into a collection of desired computations.

This article proposes a theory that leads to a novel characterization of runs encoded in concurrent systems, which is specifically suitable for analysis purposes. Intuitively, we propose to 'untie' Petri net models of concurrent systems into their untanglings - collections of abstract models capturing different portions of the behavior of the original system. Precisely, an untangling of a concurrent system is a set of its processes [Pet77, NPW81, GR83], i.e., conflict-free Petri nets, such that each process represents a (possibly infinite) subset of runs of the original system. A representative untangling of a net system describes the exact behavior of the net system, is usually larger than the net system (with respect to model size), but is easier to analyze. Representative untanglings provide a unique balance between the time required for extraction of various behavioral properties and the space required to describe concurrent systems.

This article discusses a baseline algorithm for building representative untanglings of Petri net models of concurrent systems and presents results on its termination and correctness analysis. The baseline algorithm requires an input net system to be bounded, i.e., to have a finite number of reachable states. No restrictions are put on the Petri net class; we do not require that net systems are captured as workflow nets [vdA97] or free-choice nets [DE95]. Furthermore, the article presents an improvement of the baseline algorithm that can be used to efficiently construct representative untanglings with small space footprints. This improvement is designed as a framework consisting of one or more structural transformations of input net systems and untanglings produced by the baseline algorithm. In particular, we discuss two reduction rules, inspired by Murata's rules [Mur89], that fit the framework and demonstrate that the properties of representative untanglings are all preserved by the improved algorithm.

To prove the feasibility of the proposed approach, we implemented the improved untangling algorithm and measured its performance in terms of time and space using a large collection of net systems from practice. The results confirm that representative untanglings can be constructed efficiently (in the order of a few milliseconds) and that the duplication factor w.r.t. the original net system is negligible (a representative untangling is on average only 3.5 times larger than the original system). Moreover, we demonstrate the practical advantages of representative untanglings over other formalisms for describing concurrent systems when computing various behavioral properties, e.g., executability, deadlock freedom, and mutual exclusiveness.

The remainder of this article is organized as follows: The next section introduces preliminary notions that will be used to impart the findings. Section 3 proposes process set systems - abstract models capable of representing chunks of behaviors of concurrent systems. Section 4 is devoted to the main artifact which is developed in this article - a representative untangling of a concurrent system; it proposes the baseline algorithm for constructing representative untanglings. Section 5 discusses the transformation-based optimization framework and uses this framework to propose an improved untangling algorithm. The article closes with a discussion of related work, a demonstration of different applications of representative untanglings to analyzing concurrent systems, an evaluation on a collection of concurrent systems from practice, and conclusions. 


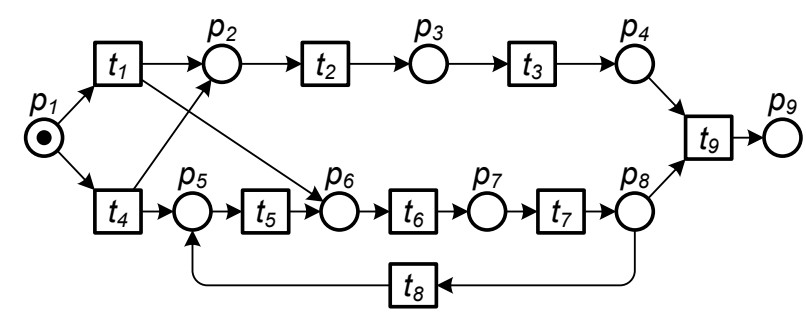

(a)

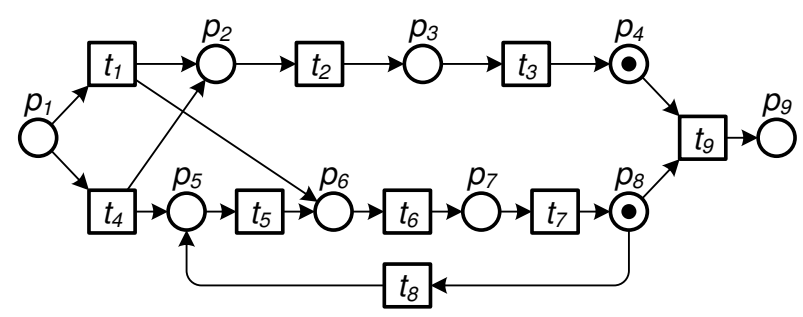

(b)

Fig. 1. Two net systems (the same net at two different markings).

\section{Preliminaries}

This section introduces formalisms that will be used to support discussions in the subsequent sections. Section 2.1 presents Petri nets and net systems - well-established formalisms for describing concurrent systems. Section 2.2 talks about processes of net systems - abstract models that are capable of describing the non-sequential behavior of concurrent systems.

\subsection{Petri Nets and Net Systems}

Petri nets are a well-known formalism for modeling concurrent systems. This section introduces the basic Petri net terminology and notations.

Definition 2.1 (Petri net). A Petri net, or a net, is a triple $N:=(P, T, F)$, where $P$ and $T$ are finite disjoint sets of places and transitions, respectively, and $F \subseteq(P \times T) \cup(T \times P)$ is the flow relation.

An element $x \in P \cup T$ is a node of $N$. A node $x \in P \cup T$ is an input node of a node $y \in P \cup T$ iff $x$ and $y$ are in the flow relation, i.e., $(x, y) \in F$. Similarly, a node $x \in P \cup T$ is an output node of a node $y \in P \cup T$ iff $(y, x) \in F$. By $\bullet x, x \in P \cup T$, we denote the preset of $x$, i.e., the set of all input nodes of $x$, $\bullet:=\{y \in P \cup T \mid(y, x) \in F\}$. Likewise, by $x \bullet, x \in P \cup T$, we refer to the postset of $x$, i.e., the set of all output nodes of $x, x \bullet:=\{y \in P \cup T \mid(x, y) \in F\}$. For a set of nodes $X \subseteq P \cup T, \bullet X:=\bigcup_{x \in X} \bullet x$ and $X \bullet:=\bigcup_{x \in X} x \bullet$. For technical convenience, we require all nets to be T-restricted. A net $N$ is T-restricted iff the preset and postset of every transition is non-empty, i.e., $\forall t \in T: \bullet t \neq \varnothing \neq t \bullet$.

A node $x \in P \cup T$ is a source node of $N$ iff $x$ has no input nodes, i.e., $\bullet x=\varnothing$. A node $x \in P \cup T$ is a $\operatorname{sink}$ node of $N$ iff $x$ has no output nodes, i.e., $x \bullet=\varnothing$. Given a net $N:=(P, T, F)$, by $\operatorname{Min}(N)$ we denote the set of all source nodes of $N$, i.e., $\operatorname{Min}(N):=\{x \in P \cup T \mid \bullet x=\varnothing\}$, whereas by $\operatorname{Max}(N)$ we denote the set of all sink nodes of $N$, i.e., $\operatorname{Max}(N):=\{x \in P \cup T \mid x \bullet=\varnothing\}$. Observe that source and sink nodes of a T-restricted net are places.

The execution semantics of a Petri net is based on the notion of a marking. A marking of a Petri net is a distribution of tokens over the net's places. Let $\mathbb{K}$ be a universe of tokens.

Definition 2.2 (Marking of a net). A marking of a net $N:=(P, T, F)$ is a pair $M:=(K, \mu)$, where $K \subseteq \mathbb{K}$ is a set of tokens and $\mu: K \rightarrow P$ is a function that assigns each token to its place.

Finally, a net system is a net at a certain marking.

Definition 2.3 (Net system).

A net system, or a system, is a pair $S:=(N, M)$, where $N$ is a net and $M$ is a marking of $N$.

In the graphical notation, a common practice is to visualize places as circles, transitions as rectangles, the flow relation as directed edges, and tokens as black dots inside the assigned places; see Fig. 1 for examples of visualizations of net systems. Both net systems in Fig. 1 are defined by the same net at different markings. The net system $(N, M), M:=(K, \mu)$, in Fig. 1(a) is at the marking that puts one token at place $p_{1}$ and no tokens elsewhere, i.e., $\mu\left(p_{1}\right)=1$ and $\mu\left(p_{i}\right)=0, i \in[2 \ldots 9]$, whereas the net system in Fig. $1(\mathrm{~b})$ is at the marking with one token at place $p_{4}$, one token at place $p_{8}$, and no tokens elsewhere. 
Whether a transition is enabled depends on tokens in its input places. An enabled transition can occur, which leads to a fresh marking.

Definition 2.4 (Semantics of a net system). ${ }^{1}$

Let $S:=(N, M), N:=(P, T, F), M:=(K, \mu)$, be a net system.

- A transition $t \in T$ is enabled in $S$, denoted by $S[t\rangle$, iff every input place of $t$ contains at least one token, i.e., $\forall p \in \bullet \exists k \in K: \mu(k)=p$.

- If a transition $t \in T$ is enabled in $S$ then $t$ can occur, which leads to a net system $S^{\prime}:=\left(N, M^{\prime}\right)$, where $M^{\prime}:=\left(K^{\prime}, \mu^{\prime}\right)$ is a marking obtained by 'consuming' one token from every input place of $t$ and 'producing' one fresh token at every output place of $t$ as follows:

Let $\hat{K} \subseteq \mathbb{K} \backslash K$ be a set of tokens such that $|\hat{K}|=|t \bullet|$ and let $g: \hat{K} \rightarrow t \bullet$ be a bijection between $\hat{K}$ and $t \bullet$. Let $f \subseteq \mu \triangleright \bullet t$, with $f$ a bijection and $|\operatorname{dom}(f)|=|\bullet t|$. Then, $K^{\prime}:=K \triangle(\operatorname{dom}(f) \cup \operatorname{dom}(g))$ and $\mu^{\prime}:=\mu \triangle(f \cup g){ }^{2}$,

The net system in Fig. 1(a) enables transitions $t_{1}$ and $t_{4}$, whereas the net system in Fig. 1(b) enables transitions $t_{8}$ and $t_{9}$. An occurrence of transition $t_{1}$ in Fig. 1(a) leads to a system with one token at place $p_{2}$, one token at $p_{6}$, and no tokens at other places. In turn, an occurrence of transition $t_{4}$ in Fig. 1(a) leads to a system with one token at place $p_{2}$, one token at place $p_{5}$, and no tokens elsewhere. By $S[t\rangle S^{\prime}$, we denote the fact that there exists an occurrence of transition $t$ that leads from $S$ to $S^{\prime}$. Observe that the execution semantics of net systems relies on holdings of tokens at places and is independent of the tokens' identities. This fact gives rise to the next relation on markings. Two markings $M_{1}:=\left(K_{1}, \mu_{1}\right)$ and $M_{2}:=\left(K_{2}, \mu_{2}\right)$ of a net are equivalent, denoted by $M_{1} \equiv M_{2}$, if and only if there exists a bijection $\beta: K_{1} \rightarrow K_{2}$ such that $\mu_{1}(k)=\left(\mu_{2} \circ \beta\right)(k), k \in K_{1}$. Clearly, the ' $\equiv$ ' relation is an equivalence relation. Every equivalence class of this relation specifies a state of the net (system), which is often identified as a multiset of the net's places.

Definition 2.5 (State of a net). The state of a net $N:=(P, T, F)$ induced by a marking $M:=(K, \mu)$, $\mu: K \rightarrow P$, is a multiset $H$ of places of $N$, where every place $p \in P$ appears $|\{k \in K \mid \mu(k)=p\}|$ times in $H$.

The state of a net system $S:=(N, M)$ is the state of $N$ induced by $M$. We shall write $\left[p_{1} p_{1} p_{2}\right]$ to denote the state induced by a marking that puts two tokens at place $p_{1}$, one token at place $p_{2}$, and no tokens elsewhere. Hence, the net system in Fig. 1(a) is at the state $\left[p_{1}\right]$, whereas the net system in Fig. $1(\mathrm{~b})$ is at $\left[p_{4} p_{8}\right]$.

Let $M_{1}$ and $M_{2}$ be two markings of a net and let $H_{1}$ and $H_{2}$ be two states of the net induced by $M_{1}$ and $M_{2}$, respectively. It is easy to see that $H_{1}=H_{2}$ iff $M_{1} \equiv M_{2}$. Let $M_{1}$ and $M_{2}$ be equivalent and let $S_{1}:=\left(N, M_{1}\right)$ and $S_{2}:=\left(N, M_{2}\right)$ be two net systems, where $N:=(P, T, F)$. Then, it holds that $\left(N, M_{1}\right)[t\rangle$ iff $\left(N, M_{2}\right)[t\rangle, t \in T$. Finally, let $\left(N, M_{1}^{\prime}\right)$ and $\left(N, M_{2}^{\prime}\right)$ be two net systems obtained after an occurrence of transition $t$ in $S_{1}$ and $S_{2}$, respectively. Clearly, it holds that $M_{1}^{\prime}$ is equivalent to $M_{2}^{\prime}$. The above observations lead to the notion of a step that describes 'equivalent' transition occurrences. A triple $\chi:=\left(H_{1}, t, H_{2}\right)$ is a step in a net $N:=(P, T, F)$, iff it holds that $\left(N, M_{1}\right)[t\rangle\left(N, M_{2}\right)$, where $H_{1}$ and $H_{2}$ are the states of net systems $\left(N, M_{1}\right)$ and $\left(N, M_{2}\right)$, respectively, and $t \in T$.

A net system induces a set of its reachable states/markings/systems.

Definition 2.6 (Run, Reachable state, Occurrence sequence).

Let $S_{0}:=\left(N, M_{0}\right), N:=(P, T, F)$, be a net system.

○ A sequence of steps $\delta:=\left(H_{0}, t_{1}, H_{1}\right)\left(H_{1}, t_{2}, H_{2}\right) \ldots$, either finite or infinite, in $N$ is a run in $S_{0}$, iff $\delta=\langle\rangle$, i.e., $\delta$ is the empty sequence, or $H_{0}$ is the state of $S_{0}$.

- A state $H$ of $N$ is reachable from $S_{0}$, denoted by $H \in\left[S_{0}\right\rangle$, iff $H$ is induced by $M_{0}$ or there exists a run $\left(H_{0}, t_{1}, H_{1}\right)\left(H_{1}, t_{2}, H_{2}\right) \ldots\left(H_{n-1}, t_{n}, H_{n}\right), n \in \mathbb{N}_{0}$, in $S_{0}$ such that $H=H_{n}$. A marking $M$ of $N$ is reachable from $S_{0}$ iff there exists a state $H \in\left[S_{0}\right\rangle$ such that $H$ is induced by $M$. A net system $(N, M)$ is reachable from $S_{0}$ iff $M$ is reachable from $S_{0}$. Similarly, we shall often refer to a state, marking, and net system that is reachable from a net system $S$ as the reachable state, marking, and net system of $S$, respectively.

- A sequence of transitions $\sigma:=t_{1} t_{2} \ldots$, either finite or infinite, of $N$ is an occurrence sequence in $S_{0}$, iff $\sigma$ is empty or there exists a run $\left(H_{0}, t_{1}, H_{1}\right)\left(H_{1}, t_{2}, H_{2}\right) \ldots$ in $S_{0}$.

\footnotetext{
1 While unusual, the use of token identities in this definition allows substantial simplification of formalisms in subsequent sections.

$2 f \triangleright X$ denotes the range restriction of function $f: Y \rightarrow Z$ to the subset of its codomain $X \subseteq Z$, i.e., $f \triangleright X:=\{(y, z) \in f \mid z \in X\}$. $\operatorname{dom}(f)$ denotes the domain of function $f$. Finally, $X \triangle Y$ denotes the symmetric difference of sets $X$ and $Y$, i.e., the union of the two sets minus their intersection: $X \triangle Y:=(X \cup Y) \backslash(X \cap Y)$.
} 
Let $S:=(N, M)$ be a net system. A step $\left(H, t, H^{\prime}\right)$ in $N$ is a step in $S$ iff $H \in[S\rangle$.

In the sequel, we shall often write $p_{1}\left[t_{1}\right\rangle p_{2} p_{6}$ to denote the step $\left(\left[p_{1}\right], t_{1},\left[p_{2} p_{6}\right]\right)$, or $p_{1}\left[t_{1}\right\rangle p_{2} p_{6}\left[t_{2}\right\rangle p_{3} p_{6}$ to refer to the sequence of steps $\left(\left[p_{1}\right], t_{1},\left[p_{2} p_{6}\right]\right)\left(\left[p_{2} p_{6}\right], t_{2},\left[p_{3} p_{6}\right]\right)$. For example, the net system in Fig. $1(\mathrm{~b})$ is reachable from the net system in Fig. 1(a), among others, via these runs:

○ $p_{1}\left[t_{1}\right\rangle p_{2} p_{6}\left[t_{2}\right\rangle p_{3} p_{6}\left[t_{6}\right\rangle p_{3} p_{7}\left[t_{7}\right\rangle p_{3} p_{8}\left[t_{3}\right\rangle p_{4} p_{8}$

○ $p_{1}\left[t_{1}\right\rangle p_{2} p_{6}\left[t_{2}\right\rangle p_{3} p_{6}\left[t_{3}\right\rangle p_{4} p_{6}\left[t_{6}\right\rangle p_{4} p_{7}\left[t_{7}\right\rangle p_{4} p_{8}$

- $p_{1}\left[t_{4}\right\rangle p_{2} p_{5}\left[t_{5}\right\rangle p_{2} p_{6}\left[t_{6}\right\rangle p_{2} p_{7}\left[t_{7}\right\rangle p_{2} p_{8}\left[t_{2}\right\rangle p_{3} p_{8}\left[t_{3}\right\rangle p_{4} p_{8}$, and

- $p_{1}\left[t_{4}\right\rangle p_{2} p_{5}\left[t_{2}\right\rangle p_{3} p_{5}\left[t_{5}\right\rangle p_{3} p_{6}\left[t_{6}\right\rangle p_{3} p_{7}\left[t_{7}\right\rangle p_{3} p_{8}\left[t_{8}\right\rangle p_{3} p_{5}\left[t_{5}\right\rangle p_{3} p_{6}\left[t_{6}\right\rangle p_{3} p_{7}\left[t_{3}\right\rangle p_{4} p_{7}\left[t_{7}\right\rangle p_{4} p_{8}$.

A net system $S:=(N, M)$ is $k$-bounded, or bounded, iff there exists a number $k \in \mathbb{N}_{0}$ such that for every marking $M$ reachable from $S$ it holds that the amount of tokens at each place of $N$ is at most $k$, which implies that the set $[S\rangle$ is finite. Observe that both net systems in Fig. 1 are bounded.

This concludes the section. For more information on Petri nets and systems, the reader can refer to [Mur89].

\subsection{Processes of Net Systems}

A common way to trace dependencies between transition occurrences in net systems is by means of runs, cf. Definition 2.6. Runs suit well when it comes to describing orderings of transition occurrences. Let $\delta:=\chi_{1} \ldots \chi_{n}, n \in \mathbb{N}_{0}$, be a run in a net system $S:=\left(N, M_{0}\right), N:=(P, T, F)$, let $H_{0}$ be the state of $S$, and let $H_{1} \ldots H_{n}$ be states of $N$ such that for every position $i$ in $\delta$ there exists $t_{i} \in T$ for which it holds that $\chi_{i}=\left(H_{i-1}, t_{i}, H_{i}\right)$. Then, every two subsequent steps $\chi_{i-1}$ and $\chi_{i}, i \in[2 . . n]$, represent two subsequent transition occurrences, such that an occurrence of $t_{i-1}$ leads to a marking that induces $H_{i-1}$ and an occurrence of $t_{i}$ takes place at a marking that induces $H_{i-1}$. However, runs of net systems cannot be used to capture such behavioral phenomena as causality and concurrency.

In this section, we discuss processes of net systems [Pet77, NPW81, GR83]. One can rely on processes to adequately represent causality and concurrency relations on transition occurrences. A process of a net system is a net of a particular kind, called a causal net (or sometimes an occurrence net, see for instance [GR83]), together with a mapping from nodes of the causal net to nodes of the net system. The mapping allows interpreting the causal net as a concurrent run of the net system.

Definition 2.7 (Causal net). A net $N:=(B, E, G)$, where $B \subseteq \mathbb{K}$, is a causal net $^{3}$, iff :

$\circ$ for every $b \in B$ it holds that $|\bullet b| \leq 1$ and $|b \bullet| \leq 1$, i.e., $N$ is conflict-free, and

$\circ G^{+}$is irreflexive, i.e., $N$ is acyclic. ${ }^{4}$

Given a causal net $N:=(B, E, G)$, elements of $B$ and $E$ are called conditions and events, respectively.

One can use events of causal nets to represent transition occurrences. Consequently, conditions in the preset and postset of an event must be interpreted as tokens that get consumed and produced, respectively, by an occurrence of the transition that corresponds to the event. Thus, we suggest that conditions of causal nets are always drawn from the universe of tokens $\mathbb{K}$. The above discussed intuition can be formalized in the notion of a process of a net system as follows.

Definition 2.8 (Process of a net system). A process of a net system $S:=\left(N, M_{0}\right), N:=(P, T, F)$, is a pair $\pi:=\left(N_{\pi}, \rho\right)$, where $N_{\pi}:=(B, E, G)$ is a causal net and $\rho: B \cup E \rightarrow P \cup T$ is such that:

○ $\rho(B) \subseteq P, \rho(E) \subseteq T$, i.e., $\rho$ preserves the nature of nodes,

- $M_{0} \equiv\left(\operatorname{Min}\left(N_{\pi}\right),\left.\rho\right|_{\operatorname{Min}\left(N_{\pi}\right)}\right)$, i.e., $\pi$ starts at $M_{0}$, and

- for every event $e \in E$ and for every place $p \in P$ it holds that $|\{(p, t) \in F \mid t=\rho(e)\}|=\left|\rho^{-1}(p) \cap \bullet e\right|$ and $|\{(t, p) \in F \mid t=\rho(e)\}|=\left|\rho^{-1}(p) \cap e \bullet\right|$, i.e., $\rho$ respects the environment of transitions.

Given a process $\pi:=\left(N_{\pi}, \rho\right), N_{\pi}:=(B, E, G)$, an event $e \in E$ represents an occurrence of transition $\rho(e)$, whereas conditions in $\bullet e$ and $e \bullet$ relate to tokens that get consumed and produced by the occurrence of $\rho(e)$. More precisely, for every condition $c \in \bullet e$ one token must be removed from place $\rho(c)$ and for every condition $c \in e \bullet$ one token must be put at place $\rho(c)$ during an occurrence of $\rho(e)$.

Fig. 2 shows three processes of the net system in Fig. 1(a). When visualizing processes, we employ elements $e_{i} e_{i}^{\prime} e_{i}^{\prime \prime} \ldots$ to denote events that refer to transition $t_{i}$ in the corresponding net system. Similarly, conditions

\footnotetext{
3 For technical convenience, we require that every element of $B$ is an element of $\mathbb{K}$.

$4 R^{+}$denotes the transitive closure of a binary relation $R$.
} 


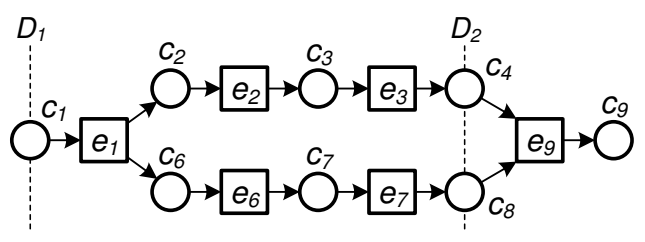

(a)

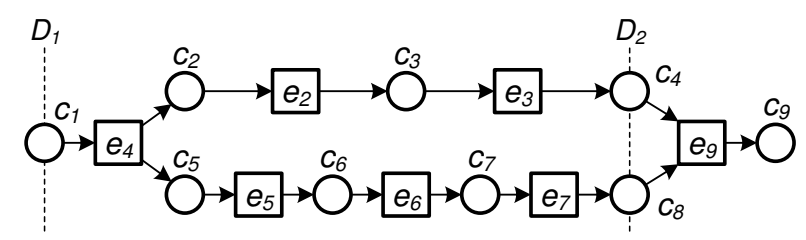

(b)

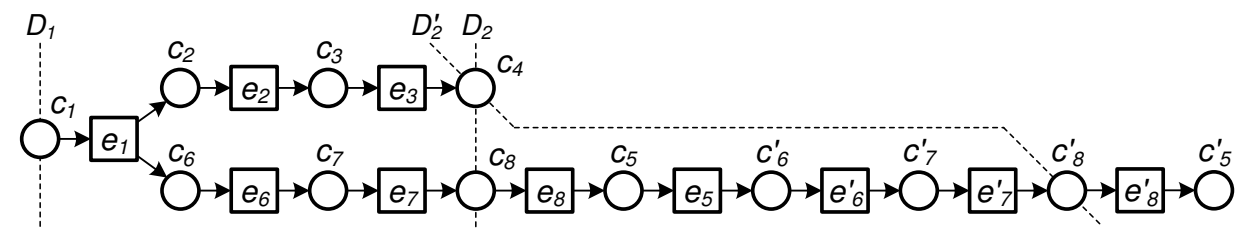

(c)

Fig. 2. Three processes of the net system in Fig. 1(a).

$c_{i} c_{i}^{\prime} c_{i}^{\prime \prime} \ldots$ are used to refer to place $p_{i}$. For example, events $e_{6}$ and $e_{6}^{\prime}$ in Fig. 2(c) refer to transition $t_{6}$ of the net in Fig. 1(a), i.e., $\rho\left(e_{6}\right)=t_{6}=\rho\left(e_{6}^{\prime}\right)$, whereas conditions $c_{6}$ and $c_{6}^{\prime}$ in Fig. 2(c) refer to place $p_{6}$, i.e., $\rho\left(c_{6}\right)=p_{6}=\rho\left(c_{6}^{\prime}\right)$. When visualizing several processes we often use identical element names, e.g., event $e_{1}$ in the processes in Figs. 2(a) and 2(c). Though it is not required, in what follows we shall always assume, for technical convenience, that elements with identical names coming from different processes are distinct. Thus, event $e_{1}$ of the process in Fig. 2(a) and event $e_{1}$ of the process in Fig. 2(c) are two different events.

Processes capture dependencies between transition occurrences as follows. Two nodes $x$ and $y$ of a causal net $N:=(B, E, G)$ are causal, or $x$ is a cause for $y$, iff $(x, y) \in G^{+}$, whereas $x$ and $y$ are concurrent iff $(x, y) \notin G^{+}$and $(y, x) \notin G^{+}$. Intuitively, two distinct events $e_{1}$ and $e_{2}$ of a causal net of a process $\left(N_{\pi}, \rho\right)$ of a net system $S$ being concurrent hints at the fact that starting from $S$ one can reach a net system $S^{\prime}$ in which transitions $\rho\left(e_{1}\right)$ and $\rho\left(e_{2}\right)$ are enabled. Moreover, an occurrence of one of the two transitions in $S^{\prime}$ leads to a net system in which the other transition is enabled. In contrast, the fact that event $e_{1}$ is a cause for event $e_{2}$ in $N_{\pi}$ indicates the presence of an order, i.e., starting from $S$ one can reach a net system $S^{\prime}$ which enables transition $\rho\left(e_{1}\right)$ such that an occurrence of $\rho\left(e_{1}\right)$ in $S^{\prime}$ leads to a net system from which one can reach a net system that enables transition $\rho\left(e_{2}\right)$. For instance, in Fig. 2(a), event $e_{1}$ is a cause for event $e_{9}$, which indicates that an occurrence of transition $t_{1}$ can act as a prerequisite for an occurrence of transition $t_{9}$ in the net system in Fig. 1(a). On the other hand, events $e_{2}$ and $e_{7}$ are concurrent, i.e., transitions $t_{2}$ and $t_{7}$ can be enabled at the same time in some net system reachable from the one shown in Fig. 1(a).

In addition to transition occurrences, processes encode reachable markings of corresponding net systems. This is accomplished by means of cuts of causal nets. A cut of a causal net is a maximal (with respect to set inclusion) set of its pairwise concurrent conditions.

Theorem 2.9 (Cuts and reachable markings, [GR83, Theorem 3.5]).

Let $\pi:=\left(N_{\pi}, \rho\right), N_{\pi}:=(B, E, G)$, be a process of a net system $S$. If $C \subseteq B$ is a cut of $N_{\pi}$, then $M:=\left(C,\left.\rho\right|_{C}\right)$ is a reachable marking of $S$.

Theorem 2.9 hints at the fact that processes of net systems can be employed as space-efficient data structures for storing reachable markings of corresponding net systems. Fig. 2(a) shows two cuts: $D_{1}$ and $D_{2}$. Each of these cuts is composed of conditions that intersect the respective dashed line. Hence, cut $D_{1}$ is equal to the singleton $\left\{c_{1}\right\}$ and cut $D_{2}$ is equal to the set of conditions $\left\{c_{4}, c_{8}\right\}$. Cuts $D_{1}$ and $D_{2}$ in Fig. 2(a) induce markings that are equivalent to markings in Fig. 1(a) and Fig. 1(b), respectively; these are markings $M_{1}:=\left(D_{1},\left.\rho\right|_{D_{1}}\right)$ and $M_{2}:=\left(D_{2},\left.\rho\right|_{D_{2}}\right)$ (note that $D_{1}$ and $D_{2}$ are sets of tokens from $\mathbb{K}$ ). Both $M_{1}$ and $M_{2}$ are indeed reachable markings of the net system in Fig. 1(a). Similarly, cuts $D_{1}$ and $D_{2}$ in Fig. 2(b) induce markings that are equivalent to those in Fig. 1(a) and Fig. 1(b), respectively. Observe that the net in Fig. 2(c) has two cuts that induce markings which are equivalent to the marking in Fig. 1(b); these are cuts $D_{2}=\left\{c_{4}, c_{8}\right\}$ and $D_{2}^{\prime}=\left\{c_{4}, c_{8}^{\prime}\right\}$, Again, similar to events and conditions of causal nets, in the rest of the 


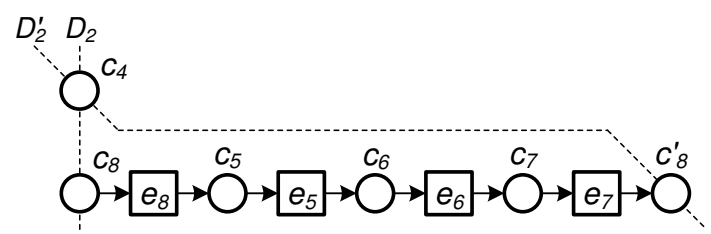

Fig. 3. A reproduction process of the net system in Fig. 1(b).

article, two cuts with the same name coming from two different processes will be considered to be different. If there is a potential for confusion, we shall explicitly mention the net that possesses the cut.

Let $\pi:=\left(N_{\pi}, \rho\right)$ be a process of a net system $S:=(N, M)$. It is known that $\operatorname{Min}\left(N_{\pi}\right)$ and $\operatorname{Max}\left(N_{\pi}\right)$ are cuts [GR83]. Moreover, by Definition 2.8, $\operatorname{Min}\left(N_{\pi}\right)$ is a cut that induces a marking that is equivalent to $M$. For example, cut $D_{1}$ in the process $\left(N_{\pi}, \rho\right)$ in Fig. $2(\mathrm{a})$ is equal to $\operatorname{Min}\left(N_{\pi}\right)$ and induces the marking that is equivalent to the marking of the net system in Fig. $1(\mathrm{a})$, i.e., $\left(D_{1},\left.\rho\right|_{D_{1}}\right)$ is equivalent to the marking with one token at place $p_{1}$ and no tokens elsewhere, which is depicted in Fig. 1(a).

This concludes the section. For more information on processes of net systems, the reader can refer to [GR83].

\section{Process (Set) Systems}

A process of a net system is often interpreted as a model that describes a finite portion of the corresponding net system's behavior. In [Des00], Jörg Desel suggests to enhance a causal net $N_{\pi}$ of a process $\pi:=\left(N_{\pi}, \rho\right)$ of a net system $S:=(N, M)$ with a marking $M_{\pi}$ that puts one token at every source place of $N_{\pi}$ and no tokens elsewhere. Then, every run in the net system $\left(N_{\pi}, M_{\pi}\right)$ 'represents' (via mapping $\rho$ ) a run in $S$. For example, consider the net system $S_{\pi}$ composed of the net in Fig. 2(a) and the marking that puts one token at condition $c_{1}$ and no tokens elsewhere. ${ }^{5}$ Then, the run $c_{1}\left[e_{1}\right\rangle c_{2} c_{6}\left[e_{2}\right\rangle c_{3} c_{6}\left[e_{6}\right\rangle c_{3} c_{7}\left[e_{7}\right\rangle c_{3} c_{8}\left[e_{3}\right\rangle c_{4} c_{8}\left[e_{9}\right\rangle c_{9}$ in $S_{\pi}$ represents the run $\rho\left(c_{1}\right)\left[\rho\left(e_{1}\right)\right\rangle \rho\left(c_{2}\right) \rho\left(c_{6}\right)\left[\rho\left(e_{2}\right)\right\rangle \rho\left(c_{3}\right) \rho\left(c_{6}\right)\left[\rho\left(e_{6}\right)\right\rangle \rho\left(c_{3}\right) \rho\left(c_{7}\right)\left[\rho\left(e_{7}\right)\right\rangle \rho\left(c_{3}\right) \rho\left(c_{8}\right)\left[\rho\left(e_{3}\right)\right\rangle \rho\left(c_{4}\right) \rho\left(c_{8}\right)\left[\rho\left(e_{9}\right)\right\rangle \rho\left(c_{9}\right)=$ $p_{1}\left[t_{1}\right\rangle p_{2} p_{6}\left[t_{2}\right\rangle p_{3} p_{6}\left[t_{6}\right\rangle p_{3} p_{7}\left[t_{7}\right\rangle p_{3} p_{8}\left[t_{3}\right\rangle p_{4} p_{8}\left[t_{9}\right\rangle p_{9}$ in the net system in Fig. $1(\mathrm{a})$. In this way, $S_{\pi}$ represents 26 different runs in the net system in Fig. 1(a). Observe that it is not required that every run leads to the state $\left[p_{9}\right]$, e.g., the process in Fig. 2(a) represents, among others, the empty run and run $p_{1}\left[t_{1}\right\rangle p_{2} p_{6}$.

The simple structure of processes (processes are static models captured as causal nets) permits simple analysis. For example, processes allow precise reasoning about causality and concurrency of transition occurrences. However, this simple analysis comes at a price, as time is traded for space. A net system can often have an infinite number of processes making any type of analysis on the set of all processes an infeasible task, e.g., both net systems in Fig. 1 induce infinitely many processes. This discussion triggers a question: Is it possible to represent the behavior of a net system with a finite number of processes? Clearly, one can give a positive answer to this question only if the space-efficiency of each individual process is 'high', i.e., it should be possible to interpret a process in such a way that it captures an infinite portion of the net system's behavior. The initial insights into the feasibility of such an interpretation of a process come from the notion of a reproduction process [BD90]. A reproduction process $\left(N_{\pi}, \rho\right)$ captures repetitive behavior as its minimal and maximal cuts, i.e., $\operatorname{Min}\left(N_{\pi}\right)$ and $\operatorname{Max}\left(N_{\pi}\right)$, induce equivalent markings.

Fig. 3 shows a reproduction process $\pi:=\left(N_{\pi}, \rho\right)$ of the net system in Fig. 1(b). Indeed, cuts $D_{2}:=$ $\operatorname{Min}\left(N_{\pi}\right)$ and $D_{2}^{\prime}:=\operatorname{Max}\left(N_{\pi}\right)$ induce equivalent markings, i.e., $\left(D_{2},\left.\rho\right|_{D_{2}}\right) \equiv\left(D_{2}^{\prime},\left.\rho\right|_{D_{2}^{\prime}}\right)$. By following the ideas from [Des00], the process in Fig. 3 enhanced with a marking that puts one token at each of its source conditions can be used to represent five runs in the net system in Fig. 1(b): the empty run, $p_{4} p_{8}\left[t_{8}\right\rangle p_{4} p_{5}$, $p_{4} p_{8}\left[t_{8}\right\rangle p_{4} p_{5}\left[t_{5}\right\rangle p_{4} p_{6}, p_{4} p_{8}\left[t_{8}\right\rangle p_{4} p_{5}\left[t_{5}\right\rangle p_{4} p_{6}\left[t_{6}\right\rangle p_{4} p_{7}$, and $p_{4} p_{8}\left[t_{8}\right\rangle p_{4} p_{5}\left[t_{5}\right\rangle p_{4} p_{6}\left[t_{6}\right\rangle p_{4} p_{7}\left[t_{7}\right\rangle p_{4} p_{8}$. The last run from the list above starts and ends at the same state, i.e., the state $\left[p_{4} p_{8}\right]$. This fact signifies that one can replay this run over and over again and, hence, interpret the process in Fig. 3 as encoding an infinite number of runs in the corresponding net system. For example, one can conclude that the process in Fig. 3 represents, among others, the run $p_{4} p_{8}\left[t_{8}\right\rangle p_{4} p_{5}\left[t_{5}\right\rangle p_{4} p_{6}\left[t_{6}\right\rangle p_{4} p_{7}\left[t_{7}\right\rangle p_{4} p_{8}\left[t_{8}\right\rangle p_{4} p_{5}\left[t_{5}\right\rangle p_{4} p_{6}$.

The process in Fig. 2(c) is not a reproduction process. However, this process has cuts that induce equivalent

\footnotetext{
$\overline{5}$ Note that tokens for markings of $N_{\pi}$ must be drawn from $\mathbb{K} \backslash B$, where $B$ is the set of conditions of $N_{\pi}$.
} 
markings, e.g., cuts $D_{2}$ and $D_{2}^{\prime}$. Some other pairs of cuts that induce equivalent markings and are not explicitly shown in Fig. 2(c) are $\left\{c_{2}, c_{6}\right\},\left\{c_{2}, c_{6}^{\prime}\right\}$ and $\left\{c_{3}, c_{7}\right\},\left\{c_{3}, c_{7}^{\prime}\right\}$. In what follows, we use this observation to propose a new interpretation for processes of net systems. Our new interpretation maximizes the portion of the corresponding net system's behavior that processes represent. We formalize this interpretation in the notion of a process system, which is discussed in detail in Section 3.1. Then, Section 3.2 proposes process set systems that generalize process systems to interpret behaviors encoded in sets of processes.

\subsection{Process Systems}

Given a process $\pi:=\left(N_{\pi}, \rho\right)$ of a net system $S$, one can 'explain' the behavior described in $\pi$ in terms of a 'restricted' semantics of $S$. That is, instead of enhancing $N_{\pi}$ with a marking, as suggested in [Des00], we propose that the somewhat modified 'token game' is played, as usual, in $S$. Formally, we implement the above intuition by means of process systems.

Definition 3.1 (Process system). A process system is a triple $\mathcal{S}_{\pi}:=(N, M, \pi)$, where $N$ is a net, $\pi$ is a process of a net system $\left(N, M^{\prime}\right)$, and $M, M^{\prime}$ are two markings of $N$.

We say that $\mathcal{S}_{\pi}:=(N, M, \pi), \pi:=\left(N_{\pi}, \rho\right)$, is a process system of the net system $(N, M)$ induced by $\pi$ if and only if $M \equiv\left(\operatorname{Min}\left(N_{\pi}\right),\left.\rho\right|_{\operatorname{Min}\left(N_{\pi}\right)}\right)$, i.e., $\pi$ starts at $M$, refer to Definition 2.8. The execution semantics of a process system - similarly to the execution semantics of a net system, cf. Definition $2.4-$ consists of the transition enablement and transition occurrence rules. The enablement rule of a net system $(N, M)$ depends on the structure of the net, i.e., on tokens in presets of transitions of $N$. In contrast, the enablement rule of a process system $(N, M, \pi), \pi:=\left(N_{\pi}, \rho\right)$, relies on the structure of $N_{\pi}$; specifically, on cuts and events of $N_{\pi}$. The exact formulation of the enablement rule is due to Theorem 2.9 and the next result.

Proposition 3.2 (Process restricted transition enablement). Let $\pi:=\left(N_{\pi}, \rho\right), N_{\pi}:=(B, E, G)$, be a process of a net system $S:=(N, M)$, let $D \subseteq B$ be a set of conditions, and let $e \in E$ be an event. If $\bullet e \subseteq D$, then transition $t:=\rho(e)$ is enabled in $\left(N, M^{\prime}\right)$, where $M^{\prime}:=\left(D,\left.\rho\right|_{D}\right)$, i.e., it holds that $\left(N, M^{\prime}\right)[t\rangle$.

Proposition 3.2 follows immediately from the fact that $\rho$ preserves the nature of nodes and environment of transitions, cf. Definition 2.8. Finally, we propose the execution semantics of a process system.

Definition 3.3 (Semantics of a process system).

Let $\mathcal{S}_{\pi}:=(N, M, \pi), N:=(P, T, F), \pi:=\left(N_{\pi}, \rho\right), N_{\pi}:=(B, E, G)$, be a process system.

- A transition $t \in T$ is enabled in $\mathcal{S}_{\pi}$, denoted by $\mathcal{S}_{\pi}[t\rangle$, iff there exist a cut $C \subseteq B$ of $N_{\pi}$ and an event $e \in E$ such that $M \equiv\left(C,\left.\rho\right|_{C}\right), \bullet e \subseteq C$, and $t=\rho(e)$.

- If a transition $t \in T$ is enabled in $\mathcal{S}_{\pi}$ then $t$ can occur, which leads to a process system $\left(N, M^{\prime}, \pi\right)$, where $M^{\prime}$ is a marking of $N$ for which it holds that $(N, M)[t\rangle\left(N, M^{\prime}\right)$.

Intuitively, the semantics of a process system $(N, M, \pi), N:=(P, T, F), \pi:=\left(N_{\pi}, \rho\right)$, is obtained by 'restricting' the semantics of the net system $(N, M)$ to those markings that are induced by cuts of $N_{\pi}$ and to those transition occurrences that are described by events of $N_{\pi}$. Let $\mathcal{S}_{\pi}$ and $\mathcal{S}_{\pi}^{\prime}$ be two process systems. By $\mathcal{S}_{\pi}[t\rangle \mathcal{S}_{\pi}^{\prime}$, we denote the fact that there exists an occurrence of transition $t \in T$ that leads from $\mathcal{S}$ to $\mathcal{S}^{\prime}$. Let $\mathcal{S}_{0}:=(N, M, \pi), N:=(P, T, F)$, be a process system. Similar to net systems, we say that the state $H$ of $N$ induced by $M$ is the state of $\mathcal{S}_{0}$. A sequence of steps $\delta:=\left(H_{0}, t_{1}, H_{1}\right)\left(H_{1}, t_{2}, H_{2}\right) \ldots$, either finite or infinite, is a run in $\mathcal{S}_{0}$, iff $\delta$ is empty or there exists a sequence of process systems $\mathcal{S}_{1} \mathcal{S}_{2} \ldots$ such that for every position $i$ in $\delta$ it holds that $\mathcal{S}_{i-1}\left[t_{i}\right\rangle \mathcal{S}_{i}$ and $H_{i-1}$ and $H_{i}$ are the states of $\mathcal{S}_{i-1}$ and $\mathcal{S}_{i}$, respectively. The notions of a reachable state, reachable marking, reachable (process) system, and that of an occurrence sequence in a (process) system are defined (mutatis mutandis) as the respective notions for net systems, cf. Definition 2.6; the only difference is that they rely on the notion of a run in a process system instead of that in a net system.

As an example, consider the process system $\mathcal{S}_{\pi}$ of the net system $(N, M)$ in Fig. 1(a) induced by process $\pi$ in Fig. 2(c). Process system $\mathcal{S}_{\pi}^{\prime}:=\left(N, M^{\prime}, \pi\right)$, where $S^{\prime}:=\left(N, M^{\prime}\right)$ is the net system in Fig. 1(b), is reachable from $\mathcal{S}_{\pi}$, e.g., via the run $p_{1}\left[t_{1}\right\rangle p_{2} p_{6}\left[t_{2}\right\rangle p_{3} p_{6}\left[t_{6}\right\rangle p_{3} p_{7}\left[t_{7}\right\rangle p_{3} p_{8}\left[t_{3}\right\rangle p_{4} p_{8}$. The net system in Fig. $1(\mathrm{~b})$ enables transitions $t_{8}$ and $t_{9} . \mathcal{S}_{\pi}^{\prime}$ restricts the semantics of $S^{\prime}$ by enabling transition $t_{8}$ only. Indeed, there exist two cuts of the causal net in Fig. 2(c) that induce markings equivalent to $M^{\prime}$; these are cuts $D_{2}$ and $D_{2}^{\prime}$. Further, there exist exactly two events $e_{8}$ and $e_{8}^{\prime}$ for which it holds that $\bullet e_{8} \subseteq D_{2}$ and $\bullet e_{8}^{\prime} \subseteq D_{2}^{\prime}$. Finally, note that events $e_{8}$ and $e_{8}^{\prime}$ both refer to transition $t_{8}$. An occurrence of transition $t_{8}$ in $\mathcal{S}_{\pi}^{\prime}$ leads to the process system 
$\mathcal{S}_{\pi}^{\prime \prime}:=\left(N, M^{\prime \prime}, \pi\right)$, where $M^{\prime \prime}$ induces the state $\left[p_{4} p_{5}\right]$. Observe that $\mathcal{S}_{\pi}^{\prime \prime}$ enables transition $t_{5}$ by means of the cut $\left\{c_{4}, c_{5}\right\}$ and event $e_{5}$ of the causal net in Fig. 2(c).

It is easy to see that the process system $\mathcal{S}_{\pi}$ of the net system in Fig. 1(a) induced by the process in Fig. 2(c) describes infinite runs (occurrence sequences), which in turn signifies that there exist infinitely many runs (occurrence sequences) in $\mathcal{S}_{\pi}$. For example, the regular expression $t_{1} t_{2} t_{6} t_{7} t_{3}\left(t_{8} t_{5} t_{6} t_{7}\right)^{*}$ matches an infinite number of occurrence sequences in $\mathcal{S}_{\pi} \cdot{ }^{6}$

\subsection{Process Set Systems}

A process system usually captures only part of the behavior of the corresponding net system. For example, the process system of net system $S$ in Fig. 1(a) induced by the process in Fig. 2(c) does not represent any run in $S$ that describes at least one occurrence of transition $t_{9}$. In order to overcome this limitation, we introduce a process set system that describes behavior of a net system restricted by a collection of processes, rather than by a single process.

Definition 3.4 (Process set system). A process set system is a triple $\mathcal{S}:=(N, M, \Pi)$, where $N$ is a net, $\Pi$ is a set of processes, or an untangling, of a net system $\left(N, M^{\prime}\right)$, and $M, M^{\prime}$ are two markings of $N$.

Again, we say that $\mathcal{S}:=(N, M, \Pi)$ is a process set system of the net system $(N, M)$ induced by $\Pi$ if and only if every process in $\Pi$ starts at $M$, refer to Definition 2.8 .

The semantics of a process set system is 'composed' of the semantics of several process systems.

Definition 3.5 (Semantics of a process set system).

Let $\mathcal{S}:=(N, M, \Pi), N:=(P, T, F)$, be a process set system.

- A transition $t \in T$ is enabled in $\mathcal{S}$, denoted by $\mathcal{S}[t\rangle$, iff there exists a process $\pi \in \Pi$ for which it holds that $(N, M, \pi)[t\rangle$.

- If a transition $t \in T$ is enabled in $\mathcal{S}$ then $t$ can occur, which leads to a process set system $\left(N, M^{\prime}, \Pi^{\prime}\right)$, where $M^{\prime}$ is a marking of $N$ for which it holds that $(N, M)[t\rangle\left(N, M^{\prime}\right)$ and $\Pi^{\prime}:=\{\pi \in \Pi \mid(N, M, \pi)[t\rangle\}$.

Let $\mathcal{S}$ and $\mathcal{S}^{\prime}$ be two process set systems. By $\mathcal{S}[t\rangle \mathcal{S}^{\prime}$, we denote the fact that there exists an occurrence of transition $t \in T$ that leads from $\mathcal{S}$ to $\mathcal{S}^{\prime}$. Let $\mathcal{S}_{0}:=(N, M, \Pi), N:=(P, T, F)$, be a process set system. Similar to net systems and process systems, the state $H$ of $N$ induced by $M$ is the state of $\mathcal{S}_{0}$. All the standard notions are lifted to process set systems accordingly. A sequence of steps $\delta:=\left(H_{0}, t_{1}, H_{1}\right)\left(H_{1}, t_{2}, H_{2}\right) \ldots$, either finite or infinite, is a run in $\mathcal{S}_{0}$, iff $\delta$ is empty or there exists a sequence of process set systems $\mathcal{S}_{1} \mathcal{S}_{2} \ldots$ such that for every position $i$ in $\delta$ it holds that $\mathcal{S}_{i-1}\left[t_{i}\right\rangle \mathcal{S}_{i}$ and $H_{i-1}$ and $H_{i}$ are the states of $\mathcal{S}_{i-1}$ and $\mathcal{S}_{i}$, respectively. Again, the notions of a reachable state, reachable marking, reachable (process set) system, and occurrence sequence in a (process set) system are defined in the same way as to those for net systems, cf. Definition 2.6, but considering runs in process set systems.

As an example, consider the process set system $\mathcal{S}$ of the net system $(N, M)$ in Fig. 1(a) induced by the set of processes $\Pi:=\left\{\pi_{1}, \pi_{2}, \pi_{3}\right\}$, where $\pi_{1}, \pi_{2}$, and $\pi_{3}$ are the processes in Fig. 2(a), Fig. 2(b), and Fig. 2(c), respectively. The process set system $\mathcal{S}$ enables transitions $t_{1}$ and $t_{4}$. Transition $t_{1}$ is enabled via cuts $D_{1}$ and events $e_{1}$ in processes $\pi_{1}$ and $\pi_{3}$. Transition $t_{4}$ is enabled via cut $D_{1}$ and event $e_{4}$ in process $\pi_{2}$. An occurrence of transition $t_{1}$ in $\mathcal{S}$ leads to the process set system $\mathcal{S}^{\prime}:=\left(N, M^{\prime}, \Pi^{\prime}\right)$, where $M^{\prime}$ induces the state $\left[p_{2} p_{6}\right]$ and $\Pi^{\prime}:=\left\{\pi_{1}, \pi_{3}\right\}$; note that $\Pi^{\prime}$ does not contain process $\pi_{2}$ as it was not used to enable $t_{1}$. From $\mathcal{S}^{\prime}$ one can reach, e.g., via the run $p_{2} p_{6}\left[t_{2}\right\rangle p_{3} p_{6}\left[t_{3}\right\rangle p_{4} p_{6}\left[t_{6}\right\rangle p_{4} p_{7}\left[t_{7}\right\rangle p_{4} p_{8}$, the process set system $\mathcal{S}^{\prime \prime}:=\left(N, M^{\prime \prime}, \Pi^{\prime}\right)$, where $M^{\prime \prime}$ induces the state $\left[p_{4} p_{8}\right] . \mathcal{S}^{\prime \prime}$ enables transitions $t_{8}$ and $t_{9}$. An occurrence of transition $t_{9}$ in $\mathcal{S}^{\prime \prime}$ leads to the process set system $\mathcal{S}^{\prime \prime \prime}:=\left(N, M^{\prime \prime \prime},\left\{\pi_{1}\right\}\right)$, where $M^{\prime \prime \prime}$ induces the state $\left[p_{9}\right]$. Alternatively, an occurrence of transition $t_{8}$ in $\mathcal{S}^{\prime \prime}$ leads to the process set system $\overline{\mathcal{S}}:=\left(N, \bar{M},\left\{\pi_{3}\right\}\right)$, where $\bar{M}$ induces the state $\left[p_{4} p_{5}\right]$. Note that $\mathcal{S}^{\prime \prime \prime}$ does not enable any transition, while $\overline{\mathcal{S}}$ enables transition $t_{5}$.

\footnotetext{
6 In the proposed regular expression, parentheses are used to define the scope and the asterisk is used to indicate that there is zero or more of the preceding element.
} 


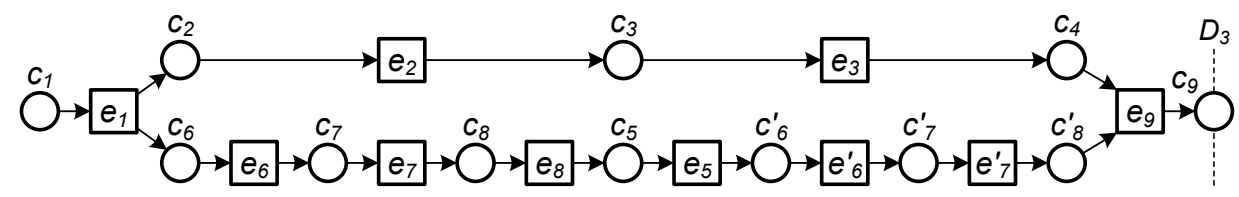

(a)

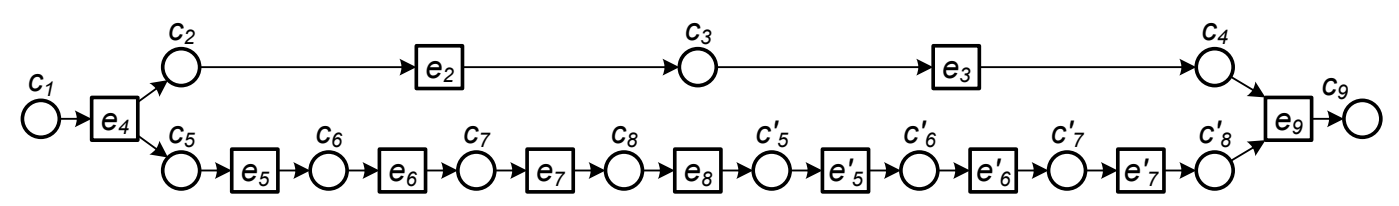

(b)

Fig. 4. A representative untangling of the net system in Fig. 1(a).

\section{Representative Untanglings}

The process set system $\mathcal{S}$ of the net system $S$ in Fig. 1(a) induced by the set of three processes in Fig. 2 represents a big share of runs in $S$, where a run $\delta$ in $S$ is considered to be represented in $\mathcal{S}$ if and only if $\delta$ is a run in $\mathcal{S}$. Still, $\mathcal{S}$ does not represent any run in $S$ in which transitions $t_{4}$ and $t_{8}$ both occur.

This section proposes the notion of a representative untangling of a net system. A representative untangling of a net system $S$ is a set of processes of $S$ that induces a process set system of $S$ that describes all the behavior of $S$ and disallows any other. Section 4.1 defines the notion of a representative untangling of a net system. Then, Section 4.2 proposes an algorithm for constructing representative untanglings.

\subsection{Definition}

A net system can be 'untangled' into a set of its processes in many different ways. Every set of processes of a net system contains information on some portion of the net system's behavior. The precise definition of this portion depends on the particular semantics that one associates with processes. If one expects to employ untangled processes for analysis, it is essential that they capture all and only the behavior of the corresponding net system (see the discussion in Section 3). Next, we characterize those untanglings that according to the semantics proposed in Section 3.2 represent the exact behavior of the corresponding systems.

Definition 4.1 (Representative untangling).

Let $\Pi$ be a set of processes, i.e., an untangling, of a net system $S:=(N, M)$.

- A process $\pi \in \Pi$ represents a step $\left(H, t, H^{\prime}\right)$ in $S$ iff it holds that $\left(N, M^{\prime}, \pi\right)[t\rangle$,

where $M^{\prime}$ is a marking of $N$ that induces $H$.

- A process $\pi \in \Pi$ represents a run $\delta$ in $S$, either finite or infinite, iff $\pi$ represents every step of $\delta$.

- An untangling $\Pi$ of $S$ is representative iff for every $\operatorname{run} \delta$ in $S$ there is a process $\pi \in \Pi$ that represents $\delta$.

Fig. 4 shows two processes of net system $S$ in Fig. 1(a) that constitute a representative untangling of $S$.

Let $\Pi$ be a representative untangling of a net system $S:=(N, M)$. Then, it holds that $S$ and the process set system $\mathcal{S}:=(N, M, \Pi)$ are in a strong behavior equivalence relation. In fact, from the point of view of an external observer, $S$ and $\mathcal{S}$ specify the same system. Both $S$ and $\mathcal{S}$ induce occurrences of transitions of $N$. Thus, whenever a transition occurs in either of the two systems, it occurs in the same environment, i.e., the same preset and postset of places. Because a run $\delta$ in $S$ is represented by some process $\pi \in \Pi$, it holds that $\delta$ is a run in $\mathcal{S}$. The converse, i.e., the result that a $\operatorname{run} \delta$ in $\mathcal{S}$ is also a run in $S$, can be obtained by appealing to Proposition 3.2 at each step of $\delta$. The above observations lead to the conclusion that $S$ and $\mathcal{S}$ are two occurrence net equivalent systems [vGV87], which is the strongest behavioral equivalence notion for models of concurrent systems [Tar97]; it states that unfoldings [McM92, McM95] of both systems are isomorphic.

A representative untangling $\Pi$ of a net system $S$ induces a process set system that allows all the behavior captured in $S$ and disallows any other. Moreover, $\Pi$ provides an alternative specification of the behavior that is described in $S$ which is particularly interesting for analysis purposes (refer to Section 7 for details); 
The unique analysis experience for $S$ that stems from the use of $\Pi$ is mainly due to the special relation between runs in $S$ and processes in $\Pi$. For example, one can test if there exists a run in $S$ with certain properties by checking if there exists a process in $\Pi$ that allows some run with these properties. Alternatively, it is possible to check if some property holds for all runs in $S$ by validating it against all processes in $\Pi$. Finally, as processes in $\Pi$ adequately represent the ordering, causality, and concurrency relations on transition occurrences, one can rely on these relations when analyzing the described behavior.

\subsection{Construction}

This section proposes an algorithm that given a bounded net system $S$ builds a representative untangling of $S$. It starts by suggesting an encoding for describing processes in pseudocode. The encoding is used to define an algorithm that given a run in a net system constructs its induced process. The algorithm for building representative untanglings relies on this construction as it proceeds by discovering those runs in the given net system that induce a set of processes with the desired characteristics. After having presented the untangling algorithm, this section closes with its termination and correctness analysis.

\subsubsection{Encoding of Processes}

One can encode processes in many different ways. For example, one can adopt the approach proposed in [ERV02]. A process can be captured as a set of nodes. A node is either a condition or an event. A condition is a pair of a place it refers to and the only event in its preset, or NIL if the condition is a source condition. An event is a pair of a transition it refers to and a set of conditions in its preset. This encoding is particularly convenient for describing nets without backward conflicts. Processes are captured as causal nets and, thus, forbid backward conflicts. However, causal nets forbid forward conflicts as well, cf. Definition 2.8. Consequently, we take a different approach and represent conditions $(B)$ and events $(E)$ as tokens and sets of tokens, respectively, i.e., $B \subseteq \mathbb{K}$ and $E \subseteq \mathcal{P}_{\geq 1}(\mathbb{K}) .^{7}$ A binary relation $\rho \subseteq(B \times P) \cup(E \times T)$ is used to specify the mapping of conditions to places $(P)$ and events to transitions $(T)$. As usual, the structure of a process is given by a flow relation $G \subseteq(B \times E) \cup(E \times B)$. This proposed process encoding follows the intuition that every condition describes a holding of a token at a certain place and every event describes an occurrence of a transition at a certain marking. Next, we implement this intuition.

\subsubsection{From Runs to Processes}

A natural way to address construction of a process is by iteratively appending fresh events to a causal net. The input to such a procedure is a run in a net system, i.e., a sequence of transition occurrences. Algorithm 1 summarizes a procedure that given a run in a net system constructs its induced process. The algorithm follows the intuition of the proof of Theorem 3.6 in [GR83].

The starting point for the construction is an 'empty' process, i.e., a process composed of conditions that correspond to tokens in the marking of the net system and no events, cf. lines 1-2 in Algorithm 1 . The construction proceeds by iteratively appending events to the process via the for loop of lines 4-11. Every appended event corresponds to a transition occurrence described by some step of the given run. Events are appended in the order in which the respective steps appear in the run. Every fresh event that gets appended to the process and has transition $t$ as its corresponding transition is appended together with output conditions that correspond to output places of $t$, refer to lines 6-9 in the algorithm. Observe that line 6 ensures that appended conditions are fresh with respect to the history of the run. The flow relation gets completed at line 8 . Note that at the start of every iteration of the for loop, the pair $(K, \mu)$ describes a marking that enables transition $t_{i}$, i.e., induces state $H_{i-1}$; refer to the input run of the algorithm.

The construction proposed in Algorithm 1 is not unique, but is always possible. Moreover, a process that results from a call to this algorithm represents the run it was constructed from.

Proposition 4.2 (Processes and runs).

If $\delta$ is a finite run in a net system $S$, then it holds that Process $(S, \delta)$ represents $\delta$.

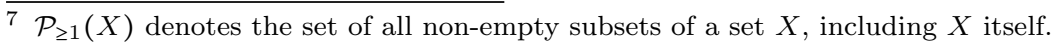




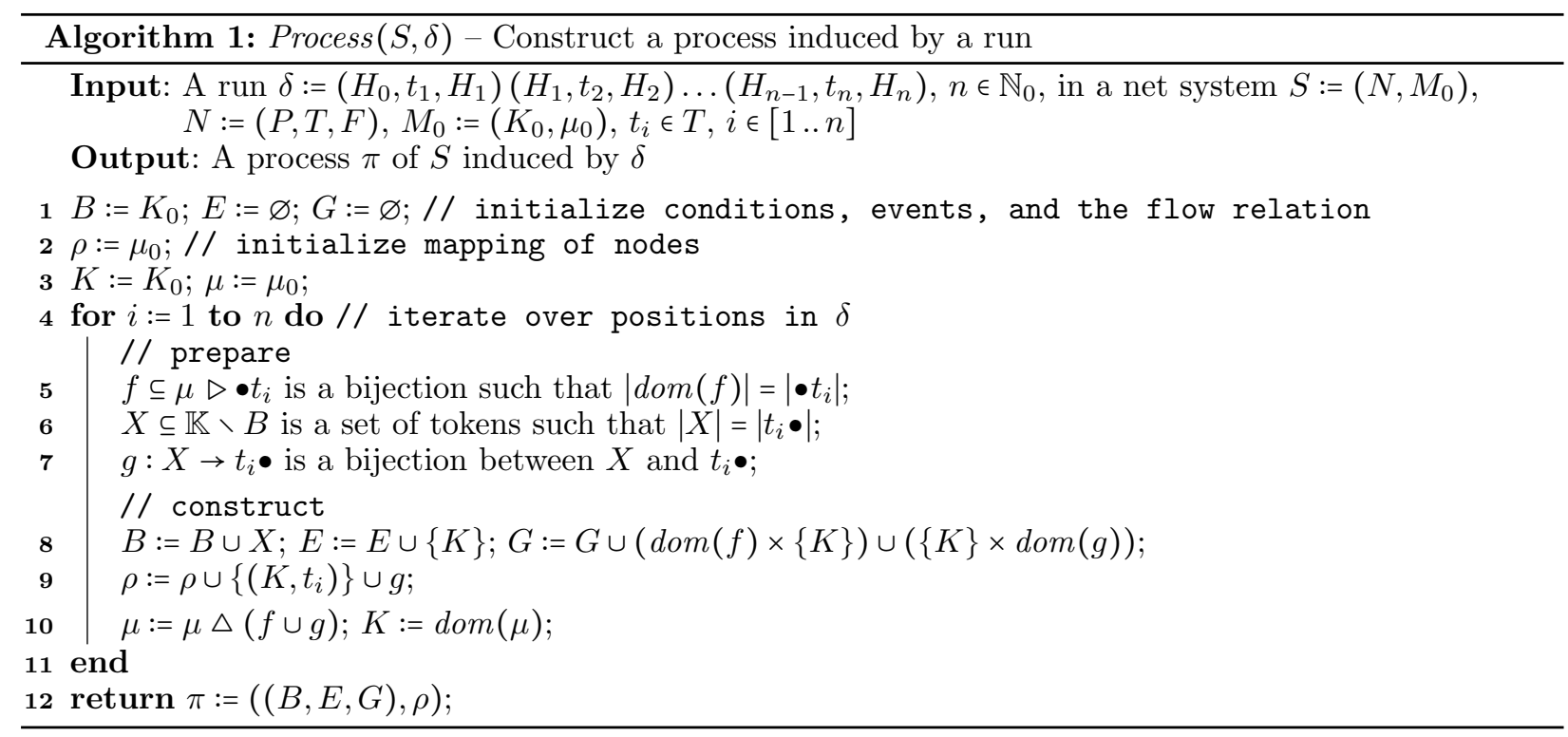

Proof. Let $\delta:=\left(H_{0}, t_{1}, H_{1}\right)\left(H_{1}, t_{2}, H_{2}\right) \ldots\left(H_{n-1}, t_{n}, H_{n}\right), n \in \mathbb{N}_{0}$, be a run in a net system $S:=(N, M)$. For every start of the $i$-th iteration of the for loop of lines $4-11$, the set $C:=\operatorname{Max}((B, E, G))$ is a cut of $(B, E, G)$ and $\left(C,\left.\rho\right|_{C}\right)$ is a marking of $N$ that induces $H_{i-1}$, cf. [GR83] for details. By the end of the $i$-th iteration of the for loop, a fresh event that corresponds to transition $t_{i}$ is appended to the process under construction with its preset completely in $C$. Hence, Process $(S, \delta)$ represents every step of $\delta$, cf. Definition 4.1 .

\subsubsection{Algorithm}

This section proposes an algorithm for constructing representative untanglings. The algorithm expects a bounded net system $S$ as input and is a state space search algorithm that discovers those runs in $S$ that induce a representative untangling of $S$. Searching a state space means systematically observing transition occurrences so as to visit all the reachable states of the net system. Subsequent transition occurrences make up runs of the net system. If one attempts to use some runs of $S$ to induce a representative untangling, one must ensure that these runs contain a sufficient amount of information on the behavior that is captured in $S$. Construction of a run can terminate when the run leads to a net system that does not enable any transition. Additionally, we propose to terminate construction of a run if it contains a subsequence that encodes a repetitive behavior that can be 'reconstructed' from other steps of the run, i.e., the run is not significant with respect to repetitive behaviors which it specifies.

Definition 4.3 (Significant run).

A run $\delta:=\chi_{1} \chi_{2} \ldots$, either finite or infinite, in a net system is repetition significant, or significant, iff:

$$
\forall i \in[1 . . n) \forall j \in(i . . n]: \chi_{i}=\chi_{j} \Rightarrow \exists k \in(i . . j) \forall m \in[1 . . i) \cup(j . . n]: \chi_{k} \neq \chi_{m} .
$$

Intuitively, a run $\delta$ is significant if every subsequence $\gamma$ of $\delta$ composed of all steps between a pair of identical steps at two distinct positions of $\delta$ is non-empty and cannot be 'reconstructed' from steps at positions of $\delta$ that are not part of $\gamma$. We shall refer to runs that are not significant as insignificant. It is easy to see that every run having length less than or equal to one is significant, whereas a run that is composed of two identical steps is insignificant. As a further example consider the sequence of steps $\delta:=p_{1}\left[t_{1}\right\rangle p_{2} p_{6}\left[t_{6}\right\rangle p_{2} p_{7}\left[t_{7}\right\rangle p_{2} p_{8}\left[t_{8}\right\rangle p_{2} p_{5}\left[t_{5}\right\rangle p_{2} p_{6}\left[t_{6}\right\rangle p_{2} p_{7}\left[t_{7}\right\rangle p_{2} p_{8}\left[t_{8}\right\rangle p_{2} p_{5}$. This sequence is a significant run in net system $S$ in Fig. 1(a). Although step $\chi:=\left(\left[p_{2} p_{6}\right], t_{6},\left[p_{2} p_{7}\right]\right)$ appears twice in $\delta$ (at positions $i=2$ and $j=6)$, observe that the step $\left(\left[p_{2} p_{5}\right], t_{5},\left[p_{2} p_{6}\right]\right)$ at position $k=5$ is unique within $\delta$ and resides between the two occurrences of $\chi$. A similar reasoning applies to steps $\left(\left[p_{2} p_{7}\right], t_{7},\left[p_{2} p_{8}\right]\right)$ and $\left(\left[p_{2} p_{8}\right], t_{8},\left[p_{2} p_{5}\right]\right)$ of $\delta$. Note, however, that sequence $\delta^{\prime}:=\delta+\left(\left[p_{2} p_{5}\right], t_{5},\left[p_{2} p_{6}\right]\right)$ is an insignificant run in $S^{8}$

\footnotetext{
$\overline{8}$ Let $\alpha$ and $\beta$ be two sequences. Then, $\alpha+\beta$ is the sequence obtained by concatenating $\alpha$ and $\beta$, i.e., joining them end-to-end.
} 


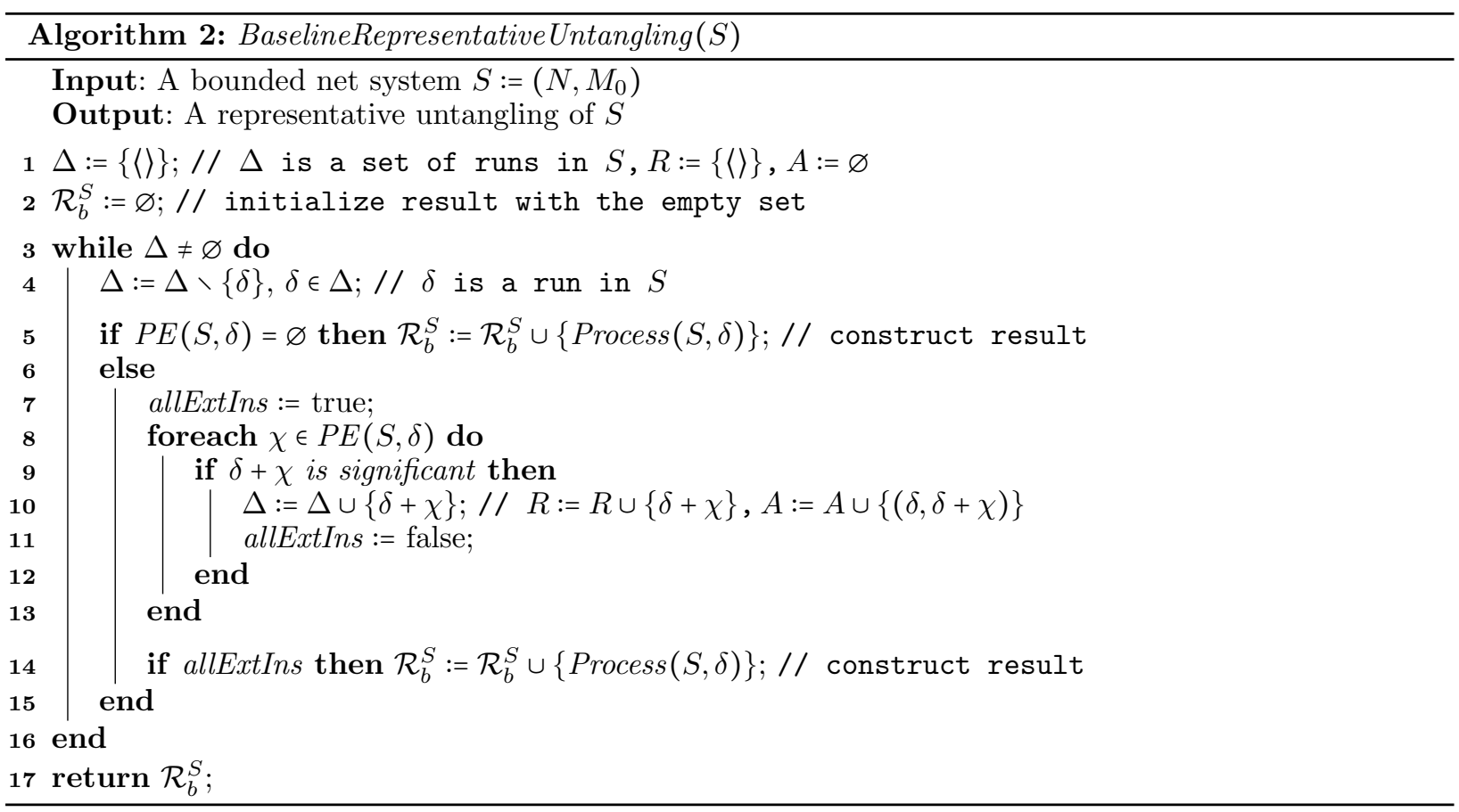

Let $\Sigma$ be the set of all steps in a net system $S$ and let $\delta:=\chi_{1} \chi_{2} \ldots \chi_{n}, n \in \mathbb{N}_{0}$, be a run in $S$. By $P E(S, \delta)$ we denote the set of all possible extensions of $\delta$, i.e., the set that contains every step $\chi \in \Sigma$ for which it holds that $\delta+\chi$ is a run in $S$. Finally, Algorithm 2 exploits the significance property of runs to construct a representative untangling of a bounded net system. Note that the code in the comments to lines 1 and 10 will be used later to reason over loop invariants.

\subsubsection{Termination Analysis}

The while loop of lines 3-16 in Algorithm 2 iterates as long as there are runs in set $\Delta$. Prior to the first iteration of the loop, at line 1 , set $\Delta$ is initialized with the empty run. At every iteration of the while loop, one run $\delta$ is drawn from $\Delta$ at line 4 . If $\delta$ has no possible extensions (see the check at line 5 ), then no runs are added to $\Delta$ in the same iteration of the loop. Otherwise, in the foreach loop of lines $8-13$, every possible extension of $\delta$ that results in a significant run (refer to the check at line 9) triggers insertion of this extended run into set $\Delta$ at line 10 . Observe that no run is added to set $\Delta$ within a single iteration of the while loop of lines 3-16 if every possible extension of $\delta$ leads to an insignificant run in $S$.

Given a bounded net system as input, Algorithm 2 systematically explores its runs. Let $R$ be a set that collects runs that are added to set $\Delta$ at lines 1 and 10 along a course of execution of Algorithm 2 - a set of visited runs; $R$ can be constructed as specified in the comments of the applicable lines of the algorithm (if executed). It is easy to see that the while loop of lines 3-16 maintains the next invariant.

Invariant 4.4 (Fresh runs - the while loop of lines 3-16).

Let $R$ be the set of visited runs up to the current execution point in Algorithm 2. Prior to every execution of line 10, it holds that the run $\delta+\chi$ is not in $R$.

Proof. Assume that before some execution of line 10, it holds that $\delta+\chi$ is in $R$. Then, in the trace of the same execution of Algorithm 2 one can observe two iterations of the while loop of lines $3-16$ that select the same run as $\delta$ at line 4 . This means that there exists an iteration of the while loop in the course of this execution such that before line 10 it holds that $\delta$ is in $R$. By applying the above reasoning iteratively, one can conclude that there exist two iterations of the while loop of lines $3-16$ that select the empty run as $\delta$ at line 4 . However, one can clearly see from the structure of the algorithm that the empty run is always selected at line 4 during the first iteration of the while loop and never again at any subsequent iteration. 
The logic of Algorithm 2 makes it apparent that its execution will never terminate if the input net system has an infinite significant run. Next, we show that there are no infinite significant runs in a bounded net system.

Lemma 4.5 (Infinite runs).

An infinite run in a bounded net system is insignificant.

Proof. Let $\delta:=\chi_{1}, \chi_{2} \ldots$ be an infinite run in a bounded net system $S:=(N, M), N:=(P, T, F)$. Assume that $\delta$ is significant. Let $\Sigma$ be the set of all steps $\left(H, t, H^{\prime}\right), t \in T$, in $S$. Because $S$ is bounded and $T$ is finite, it holds that $\Sigma$ is finite. There exists a step $\chi \in \Sigma$ such that $\chi$ occurs infinitely often in $\delta$; otherwise $\delta$ is finite. Then, there also exists an infinite sequence $\gamma$ of positions in $\delta$ (in ascending order) such that for every element $n$ in $\gamma$ it holds that $\chi=\chi_{n}$. Consequently, for every pair of subsequent elements $i$ and $j$ in $\gamma$, i.e., $i<j$, there exists $k \in(i . . j)$ such that for all $m \in[1 . . i) \cup(j . .+\infty]$ it holds that $\chi_{k} \neq \chi_{m}$; otherwise $\delta$ is insignificant. Then, it holds that $\chi$ occurs at most $|\Sigma|$ times in $\delta$ and, thus, $\gamma$ is finite.

In other words, all significant runs in a bounded net system are finite. Another threat that can cause Algorithm 2 to run forever stems from the necessity to explore an infinite number of finite significant runs. Next, we show that there is a finite number of significant runs in a bounded net system.

\section{Lemma 4.6 (Significant runs).}

The set of all significant runs in a bounded net system is finite.

Proof. Let $S:=(N, M), N:=(P, T, F)$, be a bounded net system and let $\Sigma$ be the set of all steps $\left(H, t, H^{\prime}\right)$, $t \in T$, in $S$. As $S$ is bounded and $T$ is finite, it holds that $\Sigma$ is finite. As per Lemma 4.5, every significant run in $S$ is finite. Then, there is $n \in \mathbb{N}_{0}$ such that every significant run in $S$ is a sequence of at most $n$ steps. Thus, the number of significant runs in $S$ cannot be larger than the size of a dictionary of all words of length at most $n$ such that every word is composed of symbols drawn from the fixed alphabet $\Sigma$. Such a dictionary is finite.

Finally, Theorem 4.7 collects all the above results to show that Algorithm 2 indeed terminates for every input bounded net system.

Theorem 4.7 (Termination). Algorithm 2 always terminates.

Proof. Algorithm 2 terminates if the while loop of lines 3-16 terminates. We associate a measure with every iteration of the while loop. Let $R$ be a set of visited runs that gets constructed at lines 1 and 10 in the course of some execution of Algorithm 2. Let the measure of the $i$-th iteration of the while loop be a pair $\left(\Delta_{i}, R_{i}\right)$, where $\Delta_{i}$ and $R_{i}$ are the values of $\Delta$ and $R$, respectively, at the time of the check at line 3 (prior to executing the body of the loop). For example, $\Delta_{1}$ and $R_{1}$ are both equal to $\{\langle\rangle\}$. One can classify every scenario of a single iteration of the while loop into two cases according to which relation between the measure of this iteration and the measure of the next iteration this scenario leads:

(i) $\left|\Delta_{i+1}\right|-\left|\Delta_{i}\right|=n, n \in N_{0}$, and $R_{i} \subset R_{i+1}$. This case corresponds to the scenario when a run $\delta$ chosen at line 4 has at least one significant extension and, hence, line 10 is executed one or more times during the $i$-th iteration of the while loop. Observe that both $\left|\Delta_{i}\right| \leq\left|\Delta_{i+1}\right|$ and $R_{i} \subset R_{i+1}$ hold because of Invariant 4.4.

(ii) $\Delta_{i+1} \subset \Delta_{i}$ and $R_{i}=R_{i+1}$. This case corresponds to the scenario when a run $\delta$ chosen at line 4 has no extensions or all its extensions result in insignificant runs and, thus, line 10 is not executed during the $i$-th iteration of the while loop.

The key observation here is that execution of the while loop can follow the scenario that falls under case (i) only a finite number of times. It is easy to see that the algorithm preserves the invariant of $R$ being composed of significant runs and the set of all significant runs is finite, refer to Lemma 4.6. Every time the while loop is executed according to scenario (i), the size of set $\Delta$ stays unchanged or is increased by some number $n$. Therefore, there exists an iteration of the while loop from which on every subsequent iteration of this loop will follow scenario (ii); note that at that moment in time set $\Delta$ is finite. Thus, set $\Delta$ will eventually become empty and the condition at line 3 will evaluate to false.

\subsubsection{Correctness Analysis}

Let $\delta$ be a run in a net system $S$. If an untangling $\Pi$ of $S$ is representative, then there exists a process $\pi \in \Pi$ that represents every step of $\delta$, cf. Definition 4.1. According to Proposition 4.2, a process that is constructed from a run using Algorithm 1 represents all steps of that run. Next, we show that for every run in a bounded 
net system $S$ it holds that it is composed of steps that also participate in some run that is used to induce a process in set $\mathcal{R}_{b}^{S}$ constructed using Algorithm 2.

Every fresh run that gets explored by Algorithm 2 is obtained from the one previously observed, refer to lines 4 and 10 of the algorithm. This fact gives rise to the next relation on runs.

Definition 4.8 (Graph of runs).

Let $R$ be the set of visited runs constructed by Algorithm 2 for an input bounded net system $S$. A graph of runs of $S$ is a pair $\mathcal{G}:=(R, A)$, where $A$ is the set of pairs $(\delta, \delta+\chi)$ constructed for every pair of values for $\delta$ and $\chi$ observed at line 10 during execution of Algorithm 2 for input $S$.

The principles for constructing sets $R$ and $A$ are specified in the comments to lines 1 and 10 of Algorithm 2 . Next, we point out two interesting invariants of the while loop of lines 3-16 that relate to graphs of runs.

Invariant 4.9 (Tree of runs - the while loop of lines 3-16).

Let $\Delta_{i}, \mathcal{R}_{n, i}^{S}$, and $\mathcal{G}_{i}:=\left(R_{i}, A_{i}\right)$, be the values for $\Delta$, the set of runs used to induce the set of processes $\mathcal{R}_{b}^{S}$, and the graph of runs, respectively, at the start of the $i$-th iteration of the while loop of lines 3-16 in Algorithm 2. Then, these two statements hold at the start of the $i$-th iteration of the while loop:

(i) $\mathcal{G}_{i}$ is a tree, (ii) $\Delta_{i} \cup \mathcal{R}_{n, i}^{S}$ are all the leaves of the tree $\mathcal{G}_{i}$ which is rooted at \langle\rangle$\in R_{i}$ (the empty run).

Proof. We show that statements (i) and (ii) both hold prior to the first iteration of the loop, and if statements (i) and (ii) both hold before some iteration of the loop, then they also hold before the next iteration.

Initialization: It is immediate that $\mathcal{G}_{1}:=(\{\langle\rangle\}, \varnothing)$ is a tree and $\Delta_{1} \cup \mathcal{R}_{n, 1}^{S}=\{\langle\rangle\}$ are all the leaves of $\mathcal{G}_{1}$.

Maintenance: (i) Because of Invariant 4.4, for every two subsequent iterations of the while loop of lines 3-16 it holds that $\left|R_{i+1}\right|-\left|R_{i}\right|=\left|A_{i+1}\right|-\left|A_{i}\right|$. Therefore, because $\mathcal{G}_{1}$ consists of a single vertex and no edges, it holds that $\left|A_{i+1}\right|=\left|R_{i+1}\right|-1$. In addition, it clearly holds that $\mathcal{G}_{i+1}$ is connected. (ii) A run $\delta$ that is selected from $\Delta_{i}$ at line 4 is a leaf node of $\mathcal{G}_{i}$, where $\mathcal{G}_{i}$ is rooted at \langle\rangle . Note that $\delta$ is removed from $\Delta_{i+1}$ at line 4 . If $\delta$ has an extension $\chi$ that leads to a significant run, then, because of Invariant 4.4, a fresh node $\delta+\chi$ is added to $\mathcal{G}_{i+1}$ at line 10, which is a leaf node of $\mathcal{G}_{i+1}$ (run $\delta+\chi$ is also added to $\Delta_{i+1}$ ); otherwise no fresh run is added to $R_{i+1}$ and, thus, $\delta$ is a leaf node of $\mathcal{G}_{i+1}$, and it is added to $\mathcal{R}_{n, i+1}^{S}$ at line 14 .

Moreover, it is immediate to see that at every moment in the course of execution of Algorithm 2 , set $\mathcal{R}_{b}^{S}$ is composed of processes induced by maximal significant runs, where a significant run is maximal if every one of its extensions leads to an insignificant run. Indeed, a run induces a process in $\mathcal{R}_{b}^{S}$ either if it has no extensions (line 5) or all its extensions are insignificant (allExtIns flag is set to true at line 14). Next, we propose several results on the significance of runs that we shall later orchestrate to show that Algorithm 2 is correct. We start with the claim that each extension of an insignificant run leads to an insignificant run.

\section{Proposition 4.10 (Insignificance invariant).}

Let $\delta$ be an insignificant run in a net system $S$. Then, a run $\delta+\chi$, where $\chi \in P E(S, \delta)$, is insignificant.

The proof of Proposition 4.10 follows immediately from Definition 4.3.

Proposition 4.11 (Infinite and finite runs).

Let $\delta$ be an infinite run in a bounded net system $S$. Then, there exists a finite run $\delta^{\prime}$ in $S$ such that for every step $\chi$ of $\delta$ there is a step $\chi^{\prime}$ of $\delta^{\prime}$ for which it holds that $\chi=\chi^{\prime}$.

Proof. Let $\delta:=\chi_{1}, \chi_{2} \ldots$ be an infinite run in a bounded net system $S:=(N, M), N:=(P, T, F)$. Let $\Sigma$ be the set of all steps of $\delta$. Because $S$ is bounded and $T$ is finite, it holds that $\Sigma$ is finite. Let $\Omega$ be the set that for every step $\chi \in \Sigma$ contains the smallest position $i$ in $\delta$ at which $\chi$ occurs. Then, a sequence $\delta^{\prime}$ derived from $\delta$ by deleting all the elements of $\delta$ after position $\max \Omega$ is finite. Moreover, it is guaranteed that for every step $\chi$ of $\delta$ there exists a step $\chi^{\prime}$ of $\delta^{\prime}$ for which it holds that $\chi=\chi^{\prime}$.

An important observation is that for every finite insignificant run $\delta$ there exists a significant run that has all the unique steps of $\delta$ as its elements.

Lemma 4.12 (Significant and insignificant runs).

Let $\delta$ be a finite insignificant run in a net system $S$. Then, there exists a significant run $\delta^{\prime}$ in $S$ such that for every step $\chi$ of $\delta$ there is a step $\chi^{\prime}$ of $\delta^{\prime}$ for which it holds that $\chi=\chi^{\prime}$. 
Proof. Let $\delta:=\chi_{1} \ldots \chi_{n}, n \in \mathbb{N}_{0}$, be a finite insignificant run in $S$. Then, these two conditions hold: (i) there exist two positions $i$ and $j$ in $\delta$ such that $i<j$ and $\chi_{i}=\chi_{j}$, and (ii) for every $k \in(i . . j)$ there exists $m \in[1 . . i) \cup(j . . n]$ such that $\chi_{k}=\chi_{m}$. Let $\delta^{\prime}$ be a sequence obtained from $\delta$ via a reduction operation that deletes all elements of $\delta$ after position $i$ up to and including the element at position $j$, i.e., $\delta^{\prime}:=\left(\chi_{1} \ldots \chi_{i}\right)+\left(\chi_{j+1} \ldots \chi_{n}\right)$. Clearly $\delta^{\prime}$ is a run in $S$. Moreover, because of condition (ii) above, for every step $\chi$ of $\delta$ there is a step $\chi^{\prime}$ of $\delta^{\prime}$ for which it holds that $\chi=\chi^{\prime}$. Observe that the length of $\delta^{\prime}$ is strictly smaller than the length of $\delta$. Assume that $\delta^{\prime}$ is always insignificant. Then, one can construct an infinite sequence of finite insignificant runs in $S$ that starts with $\delta$ and every other run in the sequence is obtained from the previous run via the reduction operation proposed above, which is impossible.

The next corollary is an immediate consequence of Proposition 4.11 and Lemma 4.12.

Corollary 4.13 (Runs and significant runs).

Let $\delta$ be a run, either finite or infinite, in a bounded net system $S$. Then, there exists a significant run $\delta^{\prime}$ in $S$ such that for every step $\chi$ of $\delta$ there is a step $\chi^{\prime}$ of $\delta^{\prime}$ for which it holds that $\chi=\chi^{\prime}$.

Finally, Theorem 4.14 collects all the above results to demonstrate that Algorithm 2 is correct in the sense that given a bounded net system $S$ it, indeed, computes a representative untangling of $S$.

Theorem 4.14 (Correctness).

Let $S$ be a bounded net system. Then, $\mathcal{R}_{b}^{S}$ is a representative untangling of $S$.

Proof. Upon termination of Algorithm 2, it holds that $\Delta=\varnothing$ and, hence, set $\mathcal{R}_{b}^{S}$ contains processes induced by all the runs that correspond to the leaf nodes in the tree of runs $\mathcal{G}:=(R, A)$ of $S$, refer to Invariant 4.9 Therefore, for every run $\delta$ in $S$ that is composed of steps that also participate in some leaf run in $\mathcal{G}$ it holds that there exists a process in $\mathcal{R}_{b}^{S}$ that represents $\delta$, refer to Proposition 4.2. Next, we show that the above statement holds for every significant run in $S$. Observe that $R$ is composed of significant runs, see the check at line 9 of Algorithm 2, and every internal run in $\mathcal{G}$ is a subrun (a subsequence) of some leaf run in $\mathcal{G}$. Moreover, as leaf runs in $\mathcal{G}$ are maximal and because of Proposition 4.10, it holds that $R$ is the set of all significant runs in $S$. Finally, according to Corollary 4.13, it holds that every insignificant run is composed from steps of some significant run that, in turn, is composed from steps of some leaf run in $\mathcal{G}$.

\section{Transformation-based Untangling}

Algorithm 2 is primarily of theoretical value. As the name BaselineRepresentativeUntangling suggests, it should be used as a point of reference that specifies basic principles for constructing representative untanglings. In practice, this algorithm is often 'expensive' to execute as it relies on an extensive search over state spaces induced by input net systems. For example, set $\mathcal{R}_{b}^{S}$ of the net system $S$ in Fig. 1(a) comprises 352 processes! In this section, we propose an enhancement to the baseline algorithm that can yield results that suit practical applications. The enhancement is based on structural transformations of input net systems that 'inform' subsequent structural transformations of resulting untanglings. The proposed enhancement is 'orthogonal' to the baseline technique as it proposes pre- and post-processing measures to be taken in combination with Algorithm 2. To set the scene, Section 5.1 describes the overall design of the transformation-based untangling technique and develops requirements for individual transformations. Section 5.2 proposes two transformations that fit the proposed design. Finally, Section 5.3 collects all the results of this section to explain the transformation-based untangling technique.

\subsection{Design}

The proof of correctness of Algorithm 2 exploits the observation that an untangling of $S$ is representative if it represents every maximal significant run in $S$. We formalize this observation in the next statement.

Lemma 5.1 (Representative untangling). An untangling $\Pi$ of a bounded net system $S$ is representative if and only if for every maximal significant run $\delta$ in $S$ there exists a process $\pi \in \Pi$ that represents $\delta$.

The proof of Lemma 5.1 is immediate due to Definition 4.1 and the proof of Theorem 4.14. 


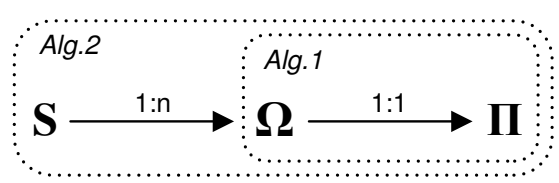

(a)

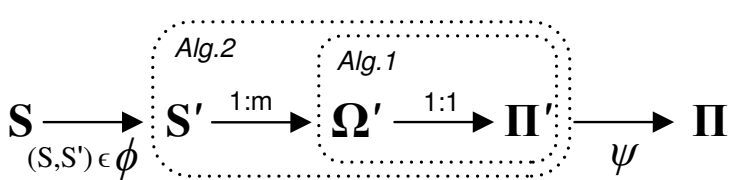

(b)

Fig. 5. Schematic visualizations of two untangling techniques: (a) Algorithm 2 only, and (b) the enhanced version of Algorithm 2 that relies on structural transformations.

Fig. 5(a) gives a schematic visualization of Algorithm 2. Starting with an input bounded net system $S$, the set $\Omega$ of all maximal significant runs in $S$ is discovered; let $n$ be the cardinality of $\Omega,|\Omega|=n$. Every maximal significant run that appears in $\Omega$ triggers construction of one process in the resulting representative untangling $\Pi$ of $S$. In the figure, boxes with dotted borderlines denote scopes of the respective algorithms.

As pointed out in the previous section, Algorithm 2 suffers from the fact that the number $n$ of all maximal significant runs in a bounded net system can be immense. Consequently, constructed representative untanglings can contain extremely large numbers of processes, which restricts their practical applicability. To address this issue, we propose an enhancement to the baseline untangling technique. Given a bounded net system $S$, we suggest that one is as far as three steps away from a representative untangling of $S$ :

1. $S$ gets transformed into a bounded net system $S^{\prime}$ using some structural transformation rule $\phi .^{9}$

2. A representative untangling $\Pi^{\prime}$ of $S^{\prime}$ is discovered by constructing processes that represent all maximal significant runs $\Omega^{\prime}$ in $S^{\prime}$, where $\left|\Omega^{\prime}\right|=m$, using Algorithm 2.

3. To compensate for the effects of the transformation at step 1 , every process in $\Pi^{\prime}$ gets transformed using some rule $\psi$ (an 'inverse' of $\phi$ ) to obtain $\Pi$ - a representative untangling of $S$.

The logic of this three-step approach is summarized in the schematic drawing in Fig. 5(b). It is designed to benefit from the decrease in the number $m$ of maximal significant runs in $S^{\prime}$ as compared to those in $S$; it is expected that $m$ is much less than $n$, i.e., $m \ll n$. It is reasonable to expect such a decrease when at step 1 of the outlined procedure one applies a structural reduction on a given net system, as many well-established structural reduction rules do indeed lead to a noticeable decrease in the number of runs in the reduced system [Mur89].

Clearly, not every pair of rules $\phi$ and $\psi$ fits the proposed algorithm design. We noticed that rules $\phi$ and $\psi$ fit the design if there exists a special relation between runs in a given net system $S$ and runs in a net system $S^{\prime}$ (the net system that $S$ is transformed into). More precisely, given a transformation $\left(S, S^{\prime}\right) \in \phi$ and a transformation rule $\psi$ between processes of $S$ and $S^{\prime}$, one can construct a representative untangling of $S$ by relying on $S^{\prime}$ and $\psi$, as suggested in Fig. $5(\mathrm{~b})$, if there exists a relation $\gamma$ between a superset $\Delta$ of all the maximal significant runs $\Omega$ in $S$ and a superset $\Lambda^{\prime}$ of all the maximal significant runs in $S^{\prime}$ such that $S, S^{\prime}, \psi$, and $\gamma$ all relate to each other in a certain way, which will be detailed below. Fig. 6 shows an augmented version of the schematic drawing in Fig. $5(\mathrm{~b}) ; \gamma$ is introduced to characterize cases when processes in $\Pi$ represent runs in $\Omega$ and, thus, all runs in $S$ (cf. Lemma 5.1).

Firstly, rule $\psi$ must correspond to the transformation $\left(S, S^{\prime}\right) \in \phi$.

Definition 5.2 (Corresponding transformation rule: Processes).

A transformation rule $\psi$ between processes of net systems corresponds to a net system transformation $\left(S, S^{\prime}\right) \in \phi$ iff for every process $\pi^{\prime}$ of $S^{\prime}$ that represents at least one maximal significant run in $S^{\prime}$ it holds that there exists a process $\pi$ of $S$ such that $\left(\pi, \pi^{\prime}\right) \in \psi$.

Intuitively, correspondence of a rule $\psi$ to a transformation $\left(S, S^{\prime}\right)$ hints at the possibility of using $\psi$ to transform every process in $\Pi^{\prime}$ into a process in $\Pi$.

Secondly, rule $\gamma$ must correspond to the transformation $\left(S, S^{\prime}\right) \in \phi$.

Definition 5.3 (Corresponding transformation rule: Runs).

A transformation rule $\gamma$ between runs in net systems corresponds to a net system transformation $\left(S, S^{\prime}\right) \in \phi$

9 A transformation rule, or a rule, $\alpha$ between two sets $A$ and $B$ is a binary relation between $A$ and $B$. A pair ( $a, b) \in \alpha$ is a transformation. The fact that $(a, b) \in \alpha$ denotes that $a$ can be transformed into $b$ using $\alpha$, and vice versa. 


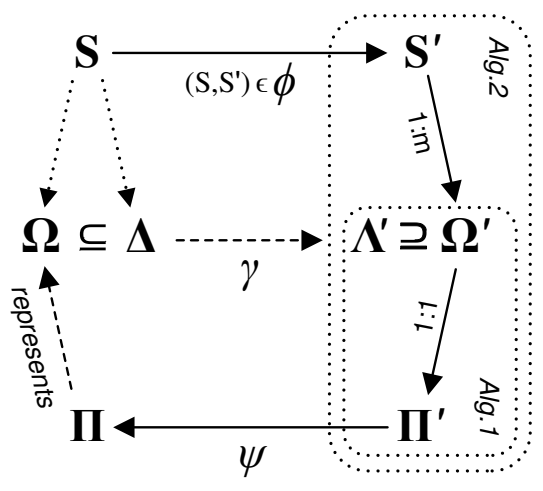

Fig. 6. Augmented version of the schematic visualization of the enhanced version of Algorithm 2 in Fig. 5(b).

iff: (i) for every maximal significant run $\delta$ in $S$ it holds that there is a run $\delta^{\prime}$ in $S^{\prime}$ such that $\left(\delta, \delta^{\prime}\right) \in \gamma$, and (ii) for all $\left(\delta, \delta^{\prime}\right) \in \gamma$ it holds that if $\delta$ is a maximal significant run in $S$ then $\delta^{\prime}$ is a significant run in $S^{\prime}$.

Intuitively, correspondence of a rule $\gamma$ to a transformation $\left(S, S^{\prime}\right)$ hints at the possibility of using $\gamma$ to transform every maximal significant run in $S$ into a significant run in $S^{\prime}$.

Finally, rules $\psi$ and $\gamma$ must be compatible.

Definition 5.4 (Compatible transformations). A transformation rule $\psi$ between processes of net systems and a transformation rule $\gamma$ between runs in net systems are compatible if and only if for all $\left(\pi, \pi^{\prime}\right) \in \psi$ and for all $\left(\delta, \delta^{\prime}\right) \in \gamma$ it holds that if $\pi^{\prime}$ represents $\delta^{\prime}$ then $\pi$ represents $\delta$.

A net system transformation rule $\phi$ is sound, iff for every $\left(S, S^{\prime}\right) \in \phi$ it holds that $S$ and $S^{\prime}$ are bounded and there exist transformation rules $\psi$ and $\gamma$ between processes of $S$ and $S^{\prime}$, and runs in $S$ and $S^{\prime}$, respectively, such that $\psi$ and $\gamma$ correspond to $\left(S, S^{\prime}\right)$, and $\psi$ and $\gamma$ are compatible.

Every transformation taken from a sound rule fits the above proposed three-step untangling approach.

Lemma 5.5 (Representativeness inheritance). Let $\left(S, S^{\prime}\right) \in \phi$ be a transformation between two bounded net systems. Let $\psi$ be a transformation rule between processes of $S$ and $S^{\prime}$ and let $\gamma$ be a transformation rule between runs in $S$ and $S^{\prime}$. Finally, let $\Pi^{\prime}$ be a representative untangling of $S^{\prime}$. If $\psi$ and $\gamma$ correspond to the transformation $\left(S, S^{\prime}\right)$ and are compatible, then $\Pi:=\psi^{-1}\left(\Pi^{\prime}\right)$ is a representative untangling of $S$.

Proof. According to Lemma 5.1, it suffices to show that $\Pi$ represents every maximal significant run in $S$. Let $\delta$ be a maximal significant run in $S$. Because $\gamma$ corresponds to the transformation $\left(S, S^{\prime}\right)$, there exists a significant $\operatorname{run} \delta^{\prime}$ in $S^{\prime}$ such that $\left(\delta, \delta^{\prime}\right) \in \gamma$. It trivially holds that there exists a maximal significant run $\delta^{\prime \prime}$ in $S^{\prime}$ such that $\delta^{\prime}$ is a subrun of $\delta^{\prime \prime} ; \delta^{\prime \prime}$ is a maximal extension of $\delta^{\prime}$ that is significant. By definition, $\Pi^{\prime}$ represents every run and, thus, every maximal significant run in $S^{\prime}$. Therefore, there exists a process $\pi^{\prime}$ of $S^{\prime}$ that represents $\delta^{\prime \prime}$ and, thus, it represents $\delta^{\prime}$. Because $\psi$ corresponds to the transformation $\left(S, S^{\prime}\right)$, there exists a process $\pi$ of $S$ such that $\left(\pi, \pi^{\prime}\right) \in \psi$. Finally, because $\psi$ and $\gamma$ are compatible, it holds that $\pi$ represents $\delta$.

A net system transformation that can be expressed as a sequence of transformations, each taken from a sound rule, also fits the above proposed design of the untangling algorithm.

Lemma 5.6 (Compositionality). Let $\left(S_{0}, S_{1}\right) \ldots\left(S_{n-1}, S_{n}\right), n \in \mathbb{N}$, be a sequence of transformations of bounded net systems. Let $\psi_{1} \ldots \psi_{n}$ and $\gamma_{1} \ldots \gamma_{n}$ be transformation rules such that for every position $i \in[1 \ldots n]$ it holds that $\psi_{i}$ and $\gamma_{i}$ are rules between processes of $S_{i-1}$ and $S_{i}$, and runs in $S_{i-1}$ and $S_{i}$, respectively, that correspond to the transformation $\left(S_{i-1}, S_{i}\right)$ and are compatible. If $\Pi_{n}$ is a representative untangling of $S_{n}$, then $\Pi_{0}:=\left(\psi_{1}^{-1} \circ \ldots \circ \psi_{n}^{-1}\right)\left(\Pi_{n}\right)$ is a representative untangling of $S_{0}$.

Proof. By induction on the length $k \in \mathbb{N}$ of a given sequence of transformations.

base: Let $\left(S_{0}, S_{1}\right)$ be a sequence of net system transformations of length $k=1$. Then, the statement holds because of Lemma 5.5 and the fact that $\psi_{1}$ and $\gamma_{1}$ correspond to $\left(S_{0}, S_{1}\right)$ and are compatible, i.e., $\Pi_{0}:=\psi_{1}^{-1}\left(\Pi_{1}\right)$ is a representative untangling of $S_{0}$, where $\Pi_{1}$ is a representative untangling of $S_{1}$. 


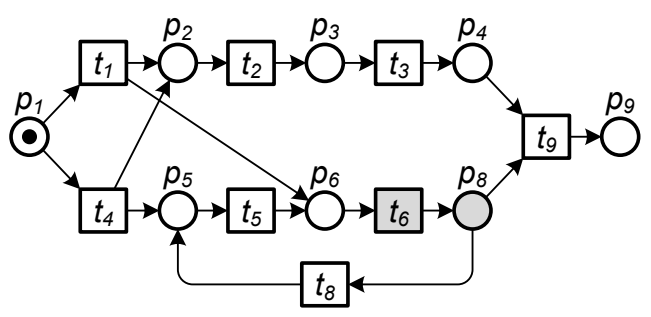

(a)

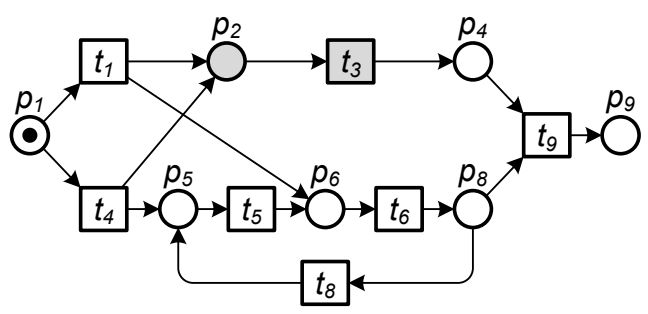

(b)

Fig. 7. (a) A net system obtained from the net system in Fig. 1(a) via the FST-reduction from $t_{6}$ to $p_{8}$, and (b) a net system obtained from the net system in Fig. 7(a) via the FSP-reduction from $p_{2}$ to $t_{3}$.

step: Assume that the statement holds for a sequence of net system transformations $\left(S_{0}, S_{1}\right) \ldots\left(S_{k-1}, S_{k}\right)$ of length $1 \leq k<n$, i.e., it holds that $\Pi_{0}:=\left(\psi_{1}^{-1} \circ \ldots \circ \psi_{k}^{-1}\right)\left(\Pi_{k}\right)$ is a representative untangling of $S_{0}$, where $\Pi_{k}$ is a representative untangling of $S_{k}$. Then, the statement must also hold for a sequence of net system transformations $\left(S_{0}, S_{1}\right) \ldots\left(S_{k}, S_{k+1}\right)$. Indeed, according to Lemma 5.5 , it holds that $\Pi_{k}^{\prime}:=\psi_{k+1}^{-1}\left(\Pi_{k+1}\right)$ is a representative untangling of $S_{k}$, where $\Pi_{k+1}$ is a representative untangling of $S_{k+1}$.

\subsection{Structural Reductions}

This section proposes two sound reduction rules on net systems. These rules allow transforming net systems into systems with fewer nodes. The names of the proposed rules are due to the names of the rules by Murata, as they are inspired by transformation rules in [Mur89].

\subsubsection{Fusion of Series Transitions}

Fusion of series transitions is a reduction rule on net systems that can be used to 'skip' a sequence of nodes composed of a place followed by a transition. The rule is defined as follows.

Definition 5.7 (Fusion of Series Transitions (FST): Net systems).

Let $S$ and $S^{\prime}$ be two net systems, where $S:=(N, M), N:=(P, T, F), M:=(K, \mu)$, and $S^{\prime}:=\left(N^{\prime}, M\right)$, $N^{\prime}:=\left(P^{\prime}, T^{\prime}, F^{\prime}\right)$. A pair $\left(S, S^{\prime}\right)$ is an FST-reduction from a transition $t \in T$ to a place $p \in P$ iff:

$\circ$ there exist a place $p^{\prime} \in P$ and a transition $t^{\prime} \in T$ such that $\bullet p^{\prime}=\{t\}, p^{\prime} \bullet=\left\{t^{\prime}\right\}, \bullet t^{\prime}=\left\{p^{\prime}\right\}, t^{\prime} \bullet=\{p\},(t, p) \notin F$, $\forall k \in K: \mu(k) \neq p^{\prime}$, and

$\circ P^{\prime}=P \backslash\left\{p^{\prime}\right\}, T^{\prime}=T \backslash\left\{t^{\prime}\right\}, F^{\prime}=\left(F \backslash\left\{\left(t, p^{\prime}\right),\left(p^{\prime}, t^{\prime}\right),\left(t^{\prime}, p\right)\right\}\right) \cup\{(t, p)\}$.

The FST-reduction rule $\phi_{A}$ on net systems consists of all FST-reductions on all net systems.

For example, a transformation $\left(S_{0}, S_{1}\right)$, where $S_{0}$ and $S_{1}$ are the net systems in Fig. 1(a) and Fig. $7($ a), respectively, is an FST-reduction between $S_{0}$ and $S_{1}$ from transition $t_{6}$ to place $p_{8}$ (highlighted with grey background in Fig. 7(a)). Given an FST-reduction $\left(S, S^{\prime}\right) \in \phi_{A}$ on net systems, the next transformation rule can be used (as it will be demonstrated later) as a rule that corresponds, see Definition 5.2 , to $\left(S, S^{\prime}\right)$.

Definition 5.8 (Fusion of Series Transitions (FST): Processes).

Let $\left(S, S^{\prime}\right) \in \phi_{A}$ be an FST-reduction on net systems, where $S:=(N, M), N:=(P, T, F)$, and $S^{\prime}:=\left(N^{\prime}, M\right)$, $N^{\prime}:=\left(P^{\prime}, T^{\prime}, F^{\prime}\right)$. Let $\pi^{\prime}:=\left(N_{\pi^{\prime}}, \rho^{\prime}\right), N_{\pi^{\prime}}:=\left(B^{\prime}, E^{\prime}, G^{\prime}\right)$, be a process of $S^{\prime}$, let $p \in P$ and $t \in T$ be such that $P^{\prime}=P \backslash\{p\}$ and $T^{\prime}=T \backslash\{t\}$, and let $\mathcal{G}:=\left\{(e, b) \in G^{\prime} \mid\left(\rho^{\prime}(e), \rho^{\prime}(b)\right) \notin F\right\}$. A pair $\left(\pi, \pi^{\prime}\right)$ is an FST-reduction to process $\pi^{\prime}$ induced by $\left(S, S^{\prime}\right)$ if and only if $\pi:=\left(N_{\pi}, \rho\right)$, where $N_{\pi}:=(B, E, G)$, is a pair such that:

$\circ B=B^{\prime} \cup\left\{b_{g} \mid g \in \mathcal{G}\right\}, E=E^{\prime} \cup\left\{e_{g} \mid g \in \mathcal{G}\right\}, G=\left(G^{\prime} \backslash \mathcal{G}\right) \cup\left\{\left(e, b_{(e, b)}\right) \mid(e, b) \in \mathcal{G}\right\} \cup\left\{\left(b_{g}, e_{g}\right) \mid g \in \mathcal{G}\right\} \cup\left\{\left(e_{(e, b)}, b\right) \mid\right.$ $(e, b) \in \mathcal{G}\}$, and

○ $\rho=\rho^{\prime} \cup\left\{\left(b_{g}, p\right) \mid g \in \mathcal{G}\right\} \cup\left\{\left(e_{g}, t\right) \mid g \in \mathcal{G}\right\}$.

The FST-reduction rule $\psi_{A}$ on processes induced by $\left(S, S^{\prime}\right)$ consists of all FST-reductions to all processes of $S^{\prime}$.,

Let $\pi$ and $\pi^{\prime}$ be processes in Fig. 2(c) and Fig. 8, respectively. Then, it holds that $\left(\pi, \pi^{\prime}\right) \in \psi_{A}$, where $\psi_{A}$ is the FST-reduction rule on processes induced by $\left(S_{0}, S_{1}\right)$. Again, $S_{0}$ and $S_{1}$ are the net systems in Fig. 1(a) 


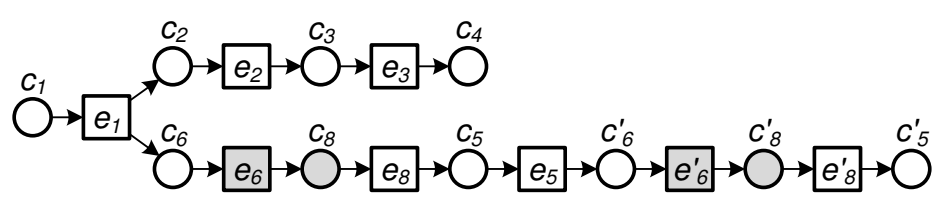

Fig. 8. A process of the net system in Fig. 7(a).

and Fig. 7(a), respectively. The reduction takes place between the nodes that are highlighted in Fig. 8. Indeed, according to Definition 5.8, an FST-reduction on processes can affect several places in the processes under transformation. Finally, we show that given an FST-reduction $\left(S, S^{\prime}\right) \in \phi_{A}$ on net systems, one can rely on rule $\psi_{A}$ on processes to transform a process of $S^{\prime}$ into a process of $S$.

Proposition 5.9 (Correctness of $\left.\psi_{A}\right)$. Let $\left(S, S^{\prime}\right) \in \phi_{A}$ be an FST-reduction on net systems. If $\pi^{\prime}$ is a process of $S^{\prime}$ and $\left(\pi, \pi^{\prime}\right) \in \psi_{A}$ is an FST-reduction on processes induced by $\left(S, S^{\prime}\right)$, then $\pi$ is a process of $S$.

Proof. (Sketch) Because $\pi^{\prime}:=\left(N_{\pi^{\prime}}, \rho^{\prime}\right), N_{\pi^{\prime}}:=\left(B^{\prime}, E^{\prime}, G^{\prime}\right)$ is a process of $S^{\prime}:=\left(N^{\prime}, M\right), N^{\prime}:=\left(P^{\prime}, T^{\prime}, F^{\prime}\right)$, and because of Definition 5.8 , it holds that $N_{\pi}$, where $\pi:=\left(N_{\pi}, \rho\right), N_{\pi}:=(B, E, G)$, is a causal net. Moreover, $\pi$ satisfies all the three criteria of a process of $S:=(N, M), N:=(P, T, F)$, cf. Definition 2.8:

$\circ\left(\rho\right.$ preserves the nature of nodes). Because $\pi^{\prime}$ is a process of $S^{\prime}$, it holds that $\rho^{\prime}$ preserves the nature of nodes. Because of Definition 5.8, it holds that $\left.\rho\right|_{\operatorname{dom}\left(\rho^{\prime}\right)}=\rho^{\prime}$. Again, because of Definition 5.8, there exist $p \in P$ and $t \in T$ such that $\forall b \in B \backslash B^{\prime}: \rho(b)=p$ and $\forall e \in E \backslash E^{\prime}: \rho(e)=t$.

$\circ(\pi$ starts at $M)$. Because of Definition 5.8, i.e., it trivially holds that $\operatorname{Min}\left(N_{\pi}\right)=\operatorname{Min}\left(N_{\pi^{\prime}}\right)$ and $\left.\rho\right|_{\operatorname{Min}\left(N_{\pi}\right)}=$ $\left.\rho^{\prime}\right|_{\operatorname{Min}\left(N_{\pi^{\prime}}\right)}$. Finally, observe that $M$ is the marking in both $S$ and $S^{\prime}$.

- $\left(\rho\right.$ respects the environment of transitions). Let $t, t^{\prime} \in T$ and $p, p^{\prime} \in P$ be such that $T=T^{\prime} \backslash\{t\}, P=P^{\prime} \backslash\{p\}$, $\left\{t^{\prime}\right\}=\bullet p$, and $\left\{p^{\prime}\right\}=t \bullet$. Assume that there exists an event $e \in E$ for which $\rho$ does not respect the environment of the corresponding transition. We consider these two cases:

1. $(\rho(e)=t)$. Because of Definitions 5.7 and 5.8, it holds that $\bullet \rho(e)=\{p\}=\rho(\bullet e)$ and $\rho(e) \bullet=\left\{p^{\prime}\right\}=\rho(e \bullet)$.

2. $(\rho(e) \neq t)$. Again, because of Definitions 5.7 and 5.8, it holds that $\bullet \rho(e)=\rho(\bullet e)$ and $\rho(e) \bullet=\rho(e \bullet)$.

Given an FST-reduction $\left(S, S^{\prime}\right) \in \phi_{A}$ on net systems, one can use the FST-reduction rule on processes induced by $\left(S, S^{\prime}\right)$ as a rule that corresponds, cf. Definition 5.2 , to $\left(S, S^{\prime}\right)$.

Lemma 5.10 (Correspondence of $\psi_{A}$ to $\left.\left(S, S^{\prime}\right) \in \phi_{A}\right)$.

Let $\left(S, S^{\prime}\right) \in \phi_{A}$ be an FST-reduction on net systems and let $\psi_{A}$ be the FST-reduction rule on processes induced by $\left(S, S^{\prime}\right)$. Then, it holds that $\psi_{A}$ corresponds to $\left(S, S^{\prime}\right)$.

Proof. By Definition 5.8, it holds that for every process $\pi^{\prime}$ of $S^{\prime}$ and, thus, for every process of $S^{\prime}$ that represents at least one maximal significant run in $S^{\prime}$, there exists $\left(\pi, \pi^{\prime}\right) \in \psi_{A}$. Moreover, according to Proposition 5.9, $\pi$ is a process of $S$.

Next, we propose a transformation rule between runs in net systems that can be used as a rule that corresponds, cf. Definition 5.3, to a given FST-reduction on net systems and is compatible, cf. Definition 5.4, with the respective rule $\psi_{A}$ on processes of net systems. We shall make several 'stops' on the way to this desired transformation. The first stop is the notion of a run adaptation rule.

Definition 5.11 (Run adaptation). Let $\left(S, S^{\prime}\right) \in \phi_{A}$ be an FST-reduction on net systems, where $S:=$ $(N, M), N:=(P, T, F)$, and $S^{\prime}:=\left(N^{\prime}, M\right), N^{\prime}:=\left(P^{\prime}, T^{\prime}, F^{\prime}\right)$. Let $p \in P$ and $t \in T$ be such that $P^{\prime}=P \backslash\{p\}$ and $T^{\prime}=T \backslash\{t\}$. Finally, let $\delta$ be a run in $S$. A pair $\left(\delta, \delta^{\prime}\right)$ is a run adaptation on $\delta$ induced by $\left(S, S^{\prime}\right)$ if and only if $\delta^{\prime}$ is a sequence of steps obtained from $\delta$ in two stages (in the specified order):

1. Construct sequence $\delta^{\prime \prime}$ from $\delta$ by removing all steps $\left(H, t^{\prime}, H^{\prime}\right)$ of $\delta$ for which it holds that $t^{\prime}=t$, without changing the order of the remaining steps.

2. Construct sequence $\delta^{\prime}$ from $\delta^{\prime \prime}$ by replacing every element $p$ with a fresh element $p^{\prime}$ in $H$ and $H^{\prime}$ of every step $\left(H, t^{\prime}, H^{\prime}\right)$ of $\delta^{\prime \prime}$, where $t \bullet=\left\{p^{\prime}\right\}$.

The run adaptation rule $\gamma_{\alpha}$ on runs induced by $\left(S, S^{\prime}\right)$ consists of all run adaptations on all runs in $S$.

Given an FST-reduction $\left(S, S^{\prime}\right) \in \phi_{A}$ on net systems, one can use $\gamma_{\alpha}$ to transform a run in $S$ into a run in $S^{\prime}$. 
Proposition 5.12 (Correctness of $\gamma_{\alpha}$ ).

Let $\left(S, S^{\prime}\right) \in \phi_{A}$ be an FST-reduction on net systems. If $\delta$ is a run in $S$ and $\left(\delta, \delta^{\prime}\right) \in \gamma_{\alpha}$ is a run adaptation induced by $\left(S, S^{\prime}\right)$, then $\delta^{\prime}$ is a run in $S^{\prime}$.

Proof. (Sketch) By induction on the length $k \in \mathbb{N}_{0}$ of $\delta$.

base: Let $\delta$ be empty, i.e., $k=0$. Then, $\delta^{\prime}$ is also empty and, thus, is a run in $S^{\prime}$.

step: Let us assume that the statement holds for $\delta:=\chi_{1} \ldots \chi_{k}, k \geq 0$, i.e., it holds that $\delta^{\prime}$ is a run in $S^{\prime}:=\left(N^{\prime}, M\right), N^{\prime}:=\left(P^{\prime}, T^{\prime}, F^{\prime}\right)$. Let $\kappa:=\delta+\chi_{k+1}$, where $\chi_{k+1}:=\left(H, t^{\prime}, H^{\prime}\right)$, be a run in $S:=(N, M)$, $N:=(P, T, F)$, and let $\left(\kappa, \kappa^{\prime}\right) \in \gamma_{\alpha}$. Finally, let $p \in P$ and $t \in T$ be such that $P^{\prime}=P \backslash\{p\}$ and $T^{\prime}=T \backslash\{t\}$. We consider these three cases:

1. $\left(t=t^{\prime}\right)$. If $t=t^{\prime}$, then because of Definition 5.11 it holds that $\kappa^{\prime}=\delta^{\prime}$ and, thus, $\kappa^{\prime}$ is a run in $S^{\prime}$.

2. $\left(t \neq t^{\prime}, p \in H\right.$, and $\left.p \notin H^{\prime}\right)$. This case is not possible as it must hold that $t=t^{\prime}$.

3. $\left(t \neq t^{\prime}\right.$, and $p \notin H$ or $\left.p \in H^{\prime}\right)$. Because of Definitions 5.7 and 5.11, it holds that $\kappa^{\prime}$ is a run in $S^{\prime}$.

The next stop towards the desired transformation on runs is a transformation that given a run $\delta$ in a net system $S$ constructs a significant run in $S$ which is composed of all distinct steps of $\delta$.

Definition 5.13 (Insignificance reduction). Let $\delta:=\chi_{1} \ldots \chi_{n}, n \in \mathbb{N}_{0}$, be a run in a net system.

○ A one step insignificance reduction (between $i$ and $j$ ) of $\delta$ is applicable if and only if there exist positions $i$ and $j$ in $\delta, i<j$, such that $\chi_{i}=\chi_{j}$, and for each position $k$ in $\delta, i<k<j$, it holds that step $\chi_{k}$ is equal to some step $\chi_{m}$ of $\delta$, where $m<i$ or $j<m$.

- An application of the reduction between $i$ and $j$ results in a fresh run $\delta^{\prime}:=\left(\chi_{1} \ldots \chi_{i}\right)+\left(\chi_{j+1} \ldots \chi_{n}\right)$.

It is easy to see that one eventually obtains a significant run by utilizing one step insignificance reductions on an input run (one after another as long as they are applicable). Observe that one may end up with different significant runs by applying different (in terms of different positions between which reductions are applied) sequences of one step insignificance reductions. By $\Lambda_{\delta}$, we denote the set of all significant runs that can be obtained from a given run $\delta$ through different sequences of one step insignificance reductions.

Considering the above, a suitable transformation rule on runs can be defined as follows.

Definition 5.14 (Run adjustment).

Let $\left(S, S^{\prime}\right) \in \phi_{A}$ be an FST-reduction on net systems and let $\delta$ be a run in $S$.

- A pair $\left(\delta, \delta^{\prime}\right)$ is a run adjustment on run $\delta$ induced by $\left(S, S^{\prime}\right)$ if and only if there exists a run $\delta^{\prime \prime}$ in $S^{\prime}$ such that $\left(\delta, \delta^{\prime \prime}\right) \in \gamma_{\alpha}$ is a run adaptation and it holds that $\delta^{\prime} \in \Lambda_{\delta^{\prime \prime}}$.

- The run adjustment rule $\gamma_{\beta}$ on runs induced by $\left(S, S^{\prime}\right)$ consists of all run adjustments on all runs in $S$.

Given an FST-reduction $\left(S, S^{\prime}\right) \in \phi_{A}$ on net systems, one can use the run adjustment rule induced by $\left(S, S^{\prime}\right)$ as a rule that corresponds, cf. Definition 5.3 , to $\left(S, S^{\prime}\right)$.

Lemma 5.15 (Correspondence of $\gamma_{\beta}$ to $\left(S, S^{\prime}\right) \in \phi_{A}$ ).

Let $\left(S, S^{\prime}\right) \in \phi_{A}$ be an FST-reduction on net systems and let $\gamma_{\beta}$ be the run adjustment rule induced by $\left(S, S^{\prime}\right)$. Then, it holds that $\gamma_{\beta}$ corresponds to $\left(S, S^{\prime}\right)$.

Proof. By Definition 5.14, it holds that for every run $\delta$ in $S$ and, thus, for every maximal significant run in $S$, there exists a run $\delta^{\prime}$ in $S^{\prime}$ such that $\left(\delta, \delta^{\prime}\right) \in \gamma_{\beta}$. According to Proposition 5.12 and Definition $5.14, \delta^{\prime}$ is a significant run in $S^{\prime}$.

The next statement claims that transformation rules $\psi_{A}$ and $\gamma_{\beta}$ are compatible, cf. Definition 5.4.

Lemma 5.16 (Compatibility of $\psi_{A}$ and $\left.\gamma_{\beta}\right)$. Let $\left(S, S^{\prime}\right) \in \phi_{A}$ be an FST-reduction on net systems, and let $\psi_{A}$ and $\gamma_{\beta}$ be the FST-reduction rule on processes induced by $\left(S, S^{\prime}\right)$ and the run adjustment rule induced by $\left(S, S^{\prime}\right)$, respectively. Then, it holds that $\psi_{A}$ and $\gamma_{\beta}$ are compatible.

Proof. (Sketch) Let us assume that $\psi_{A}$ and $\gamma_{\beta}$ are incompatible. Then, there exist $\left(\pi, \pi^{\prime}\right) \in \psi_{A}$ and $\left(\delta, \delta^{\prime}\right) \in \gamma_{\beta}$ such that $\pi^{\prime}$ represents $\delta^{\prime}$ but $\pi$ does not represent $\delta$, i.e., there exists a step $\chi:=\left(H, t, H^{\prime}\right)$ of $\delta$ that $\pi$ does not represent. Let $S:=(N, M), N:=(P, T, F), S^{\prime}:=\left(N^{\prime}, M\right), N^{\prime}:=\left(P^{\prime}, T^{\prime}, F^{\prime}\right)$, and let $p^{\prime}, p^{\prime \prime} \in P$ and $t^{\prime} \in T$ be such that $P^{\prime}=P \backslash\left\{p^{\prime}\right\}, T^{\prime}=T \backslash\left\{t^{\prime}\right\}$, and $\left\{p^{\prime \prime}\right\}=t^{\prime} \bullet$. We consider these two cases:

1. $\left(\chi\right.$ is a step of $\left.\delta^{\prime}\right)$. It follows immediately from Definition 5.8 that $\pi$ represents $\chi$.

2. ( $\chi$ is not a step of $\delta^{\prime}$ ). We consider these two subcases:

(a) (There exists a step $\chi^{\prime}$ of $\delta^{\prime}$ that represents an occurrence of $t$ ).

Let $\chi^{\prime}:=\left(Q, t, Q^{\prime}\right)$. We consider these three subcases: 
(i) $\left(H=Q, H^{\prime} \neq Q^{\prime}\right.$, and $Q^{\prime}$ can be obtained from $H^{\prime}$ by replacing every element $p^{\prime}$ with $\left.p^{\prime \prime}\right)$. Because of Definition 5.8, $\pi$ represents $\chi$. Then, there exists a cut $C$ and an event $e$ in $\pi$ such that $C$ induces $H$ and $e$ represents an occurrence of $t$.

(ii) $\left(H^{\prime}=Q^{\prime}, H \neq Q\right.$, and $Q$ can be obtained from $H$ by replacing every element $p^{\prime}$ with $\left.p^{\prime \prime}\right)$. It must hold that $t=t^{\prime}$ and, thus, because of Definition 5.14, there exists no step $\chi^{\prime}$ of $\delta^{\prime}$.

(iii) $\left(H \neq Q, H^{\prime} \neq Q^{\prime}\right.$, and $Q$ and $Q^{\prime}$ can be obtained from $H$ and $H^{\prime}$, respectively, by replacing every element $p^{\prime}$ with $p^{\prime \prime}$ ). Because of Definition 5.8, $\pi$ represents $\chi$. Then, there exists a cut $C$ and an event $e$ in $\pi$ such that $C$ induces $H$ and $e$ represents an occurrence of $t$.

(b) (There exists no step $\chi^{\prime}$ of $\delta^{\prime}$ that represents an occurrence of $t$ ). It must hold that $t=t^{\prime}$. There exists a step $\chi^{\prime}:=\left(Q, t^{*}, Q^{\prime}\right)$ of $\delta^{\prime}$ such that either $Q=H^{\prime}$ or $Q^{\prime}=H^{\prime}$. Thus, there exists a cut $C$ in $\pi^{\prime}$ that induces $H^{\prime}$. Then, there exists a cut $C^{\prime}$ in $\pi$ that induces $H^{\prime}$. Finally, because of Definition 5.8, it is easy to see that $\pi$ represents $\chi$.

We conclude this section with a claim that the FST-reduction rule on net systems is sound.

Lemma 5.17 (Soundness of $\phi_{A}$ ). The FST-reduction rule $\phi_{A}$ on net systems is sound.

Proof. Let $\left(S, S^{\prime}\right) \in \phi_{A}$ be an FST-reduction on net systems. Let $\psi_{A}$ be the FST-reduction on processes of net systems and let $\gamma_{\beta}$ be the run adjustment rule; both induced by $\left(S, S^{\prime}\right)$. According to Lemmas 5.10 and 5.15 , rules $\psi_{A}$ and $\gamma_{\beta}$ correspond to $\left(S, S^{\prime}\right)$. Finally, according to Lemma 5.16, rules $\psi_{A}$ and $\gamma_{\beta}$ are compatible.

\subsubsection{Fusion of Series Places}

Fusion of series places is a reduction rule on net systems that can be used to 'skip' a sequence of nodes composed of a transition followed by a place. The precise definition of the rule proceeds as follows.

Definition 5.18 (Fusion of Series Places (FSP): Net systems).

Let $S$ and $S^{\prime}$ be two net systems, where $S:=(N, M), N:=(P, T, F), M:=(K, \mu)$, and $S^{\prime}:=\left(N^{\prime}, M\right)$, $N^{\prime}:=\left(P^{\prime}, T^{\prime}, F^{\prime}\right)$. A pair $\left(S, S^{\prime}\right)$ is an FSP-reduction from a place $p \in P$ to a transition $t \in T$ iff :

$\circ$ there exist a place $p^{\prime} \in P$ and a transition $t^{\prime} \in T$ such that $\bullet t^{\prime}=\{p\}, t^{\prime} \bullet=\left\{p^{\prime}\right\}, \bullet p^{\prime}=\left\{t^{\prime}\right\}, p^{\prime} \bullet=\{t\}$, $(p, t) \notin F,(|p \bullet|=1$ or $|\bullet t|=1), \forall k \in K: \mu(k) \neq p^{\prime}$, and

○ $P^{\prime}=P \backslash\left\{p^{\prime}\right\}, T^{\prime}=T \backslash\left\{t^{\prime}\right\}, F^{\prime}=\left(F \backslash\left\{\left(p, t^{\prime}\right),\left(t^{\prime}, p^{\prime}\right),\left(p^{\prime}, t\right)\right\}\right) \cup\{(p, t)\}$.

The FSP-reduction rule $\phi_{B}$ on net systems consists of all FSP-reductions on all net systems.

For example, a transformation $\left(S_{1}, S_{2}\right)$, where $S_{1}$ and $S_{2}$ are the net systems in Fig. $7($ a) and Fig. $7(\mathrm{~b})$, respectively, is an FSP-reduction from place $p_{2}$ to transition $t_{3}$ (highlighted with grey background in Fig. $7(\mathrm{~b})$ ). Given an FSP-reduction $\left(S, S^{\prime}\right) \in \phi_{B}$ on net systems, the next transformation rule can be used as a rule that corresponds, see Definition 5.2 , to $\left(S, S^{\prime}\right)$.

Definition 5.19 (Fusion of Series Places (FSP): Processes).

Let $\left(S, S^{\prime}\right) \in \phi_{B}$ be an FSP-reduction on net systems, where $S:=(N, M), N:=(P, T, F)$, and $S^{\prime}:=\left(N^{\prime}, M\right)$, $N^{\prime}:=\left(P^{\prime}, T^{\prime}, F^{\prime}\right)$. Let $\pi^{\prime}:=\left(N_{\pi^{\prime}}, \rho^{\prime}\right), N_{\pi^{\prime}}:=\left(B^{\prime}, E^{\prime}, G^{\prime}\right)$, be a process of $S^{\prime}$, let $p \in P$ and $t \in T$ be such that $P^{\prime}=P \backslash\{p\}$ and $T^{\prime}=T \backslash\{t\}$, let $\mathcal{G}:=\left\{(b, e) \in G^{\prime} \mid\left(\rho^{\prime}(b), \rho^{\prime}(e)\right) \notin F\right\}$, and let $\mathcal{B}:=\left\{c \in B^{\prime} \mid c \bullet=\varnothing \wedge t \in \rho(c) \bullet\right\}$. A pair $\left(\pi, \pi^{\prime}\right)$ is an FSP-reduction to process $\pi^{\prime}$ induced by $\left(S, S^{\prime}\right)$ if and only if $\pi:=\left(N_{\pi}, \rho\right)$, where $N_{\pi}:=(B, E, G)$, is a pair such as:

$\circ B=B^{\prime} \cup\left\{b_{g} \mid g \in \mathcal{G}\right\} \cup\left\{b_{c} \mid c \in \mathcal{B}\right\}, E=E^{\prime} \cup\left\{e_{g} \mid g \in \mathcal{G}\right\} \cup\left\{e_{c} \mid c \in \mathcal{B}\right\}, G=\left(G^{\prime} \backslash \mathcal{G}\right) \cup\left\{\left(b, e_{(b, e)}\right) \mid(b, e) \in \mathcal{G}\right\}$

$\cup\left\{\left(e_{g}, b_{g}\right) \mid g \in \mathcal{G}\right\} \cup\left\{\left(b_{(b, e)}, e\right) \mid(b, e) \in \mathcal{G}\right\} \cup\left\{\left(c, e_{c}\right) \mid c \in \mathcal{B}\right\} \cup\left\{\left(e_{c}, b_{c}\right) \mid c \in \mathcal{B}\right\}$, and

○ $\rho=\rho^{\prime} \cup\left\{\left(b_{g}, p\right) \mid g \in \mathcal{G}\right\} \cup\left\{\left(e_{g}, t\right) \mid g \in \mathcal{G}\right\} \cup\left\{\left(b_{c}, p\right) \mid c \in \mathcal{B}\right\} \cup\left\{\left(e_{c}, t\right) \mid c \in \mathcal{B}\right\}$.

The FSP-reduction rule $\psi_{B}$ on processes induced by $\left(S, S^{\prime}\right)$ consists of all FSP-reductions to all processes of $S^{\prime}$.

The proof of the fact that the FSP-reduction rule $\phi_{B}$ on net systems is sound proceeds similar to the proof of Lemma 5.17 with a minor difference that it relies on rule $\psi_{B}$ on processes, cf. Definition 5.19, and rule $\gamma_{\beta}$ on runs in net systems, cf. Definition 5.14. Note that one can prove that rules $\psi_{B}$ and $\gamma_{\beta}$ correspond to an FSP-reduction on net systems and that they are compatible similar to the proofs of Lemmas 5.10, 5.15, and 5.16, changing those things that need to be changed. 


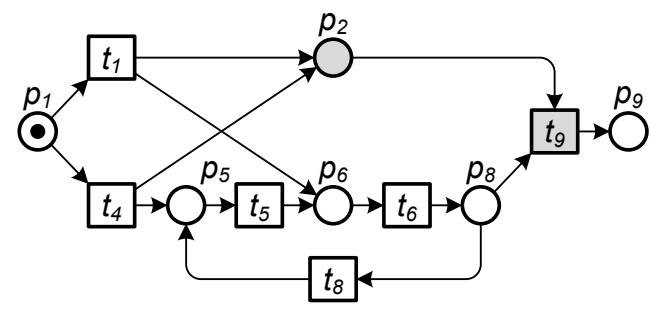

Fig. 9. A net system obtained from the net system in Fig. 7(b) via the FSP-reduction from $p_{2}$ to $t_{9}$.

\subsection{Algorithm}

This section organizes all the above proposed results in an alternative technique for computing representative untanglings. Given a repertoire, i.e., a set, of sound transformation rules $\Phi$ on net systems, the baseline construction of a representative untangling of a bounded net system $S_{0}$ can be augmented as follows. Firstly, $S_{0}$ gets transformed into a net system $S_{n}$ via a sequence of transformations $\kappa:=\left(S_{0}, S_{1}\right) \ldots\left(S_{n-1}, S_{n}\right)$, $n \in \mathbb{N}$, where each transformation belongs to some rule in $\Phi$. Secondly, a representative untangling $\mathcal{R}_{b}^{S_{n}}$ of $S_{n}$ is constructed using Algorithm 2. Finally, the set $\left(\psi_{1}^{-1} \circ \ldots \circ \psi_{n}^{-1}\right)\left(\mathcal{R}_{b}^{S_{n}}\right)$ is a representative untangling of $S_{0}$, denoted by $\mathcal{R}_{\kappa}^{S_{0}}$, where $\psi_{1} \ldots \psi_{n}$ correspond to transformations $\left(S_{0}, S_{1}\right) \ldots\left(S_{n-1}, S_{n}\right)$, respectively. Termination of the outlined algorithm is guaranteed by the finiteness of $\kappa$ and the proof of termination of Algorithm 2, whereas correctness is guaranteed by Lemma 5.6 and the proof of correctness of Algorithm 2 .

Given a bounded net system $S$, the construction of $\mathcal{R}_{\kappa}^{S}$ depends on $\kappa$, i.e., different sequences of transformations usually lead to different representative untanglings. However, it is easy to see that if one constructs $\kappa$ as long as possible by using rules from the repertoire $\Phi:=\left\{\phi_{A}, \phi_{B}\right\}$, in any order, one always ends up with the same set $\mathcal{R}_{\kappa}^{S}$ (if $\psi_{A}$ and $\psi_{B}$ are used as rules that correspond to $\phi_{A}$ and $\phi_{B}$, respectively).

Next, we exemplify the transformation-based untangling technique by constructing a representative untangling of the net system in Fig. 1(a). Firstly, one needs to come up with a sequence of transformations on the given net system, where each transformation must belong to a sound transformation rule. It is intended that the transformations reduce the net system structurally. For example, the sequence $\kappa:=\left(S_{0}, S_{1}\right)\left(S_{1}, S_{2}\right)\left(S_{2}, S_{3}\right)$, where $S_{0}, S_{1}, S_{2}$, and $S_{3}$ are the net systems in Figs. 1(a), 7(a), 7(b), and 9, respectively, is a sequence of transformations that reduces $S_{0}$ to $S_{3}$. It holds that $\left(S_{0}, S_{1}\right) \in \phi_{A}$ and $\left(S_{1}, S_{2}\right),\left(S_{2}, S_{3}\right) \in \phi_{B}$, refer to Section 5.2. Observe that $S_{3}$ is obtained from $S_{2}$ via the FSP-reduction from place $p_{2}$ to transition $t_{9}$ (highlighted with grey background in Fig. 9). Thus, all transformations of $\kappa$ belong to sound transformation rules. Secondly, a representative untangling $\mathcal{R}_{b}^{S_{3}}$ of $S_{3}$ must be constructed. We rely on Algorithm 2 to accomplish this step. Algorithm 2 proceeds by discovering all the five maximal significant runs in $S_{3}$ :

$\delta_{0}:=p_{1}\left[t_{1}\right\rangle p_{2} p_{6}\left[t_{6}\right\rangle p_{2} p_{8}\left[t_{9}\right\rangle p_{9}$,

$\delta_{1}:=p_{1}\left[t_{4}\right\rangle p_{2} p_{5}\left[t_{5}\right\rangle p_{2} p_{6}\left[t_{6}\right\rangle p_{2} p_{8}\left[t_{9}\right\rangle p_{9}$,

$\delta_{2}:=p_{1}\left[t_{1}\right\rangle p_{2} p_{6}\left[t_{6}\right\rangle p_{2} p_{8}\left[t_{8}\right\rangle p_{2} p_{5}\left[t_{5}\right\rangle p_{2} p_{6}\left[t_{6}\right\rangle p_{2} p_{8}\left[t_{9}\right\rangle p_{9}$

$\delta_{3}:=p_{1}\left[t_{4}\right\rangle p_{2} p_{5}\left[t_{5}\right\rangle p_{2} p_{6}\left[t_{6}\right\rangle p_{2} p_{8}\left[t_{8}\right\rangle p_{2} p_{5}\left[t_{5}\right\rangle p_{2} p_{6}\left[t_{6}\right\rangle p_{2} p_{8}\left[t_{9}\right\rangle p_{9}$, and

$\delta_{4}:=p_{1}\left[t_{1}\right\rangle p_{2} p_{6}\left[t_{6}\right\rangle p_{2} p_{8}\left[t_{8}\right\rangle p_{2} p_{5}\left[t_{5}\right\rangle p_{2} p_{6}\left[t_{6}\right\rangle p_{2} p_{8}\left[t_{8}\right\rangle p_{2} p_{5}$.

Algorithm 2 uses the above five runs to construct five process in $\mathcal{R}_{b}^{S_{3}}$. Finally, the set $\mathcal{R}_{\kappa}^{S_{0}}$ that is defined as $\left(\psi_{A}^{-1} \circ \psi_{B}^{-1} \circ \psi_{B}^{-1}\right)\left(\mathcal{R}_{b}^{S_{3}}\right)$, where $\psi_{A}$ and $\psi_{B}$ are transformations on processes of net systems that correspond to transformations drawn from rules $\phi_{A}$ and $\phi_{B}$, respectively (refer to Section 5.2.1 and Section 5.2.2 for details), is a representative untangling of $S_{0}$. The set $\mathcal{R}_{\kappa}^{S_{0}}$ contains the five processes shown in Figs. 2 and 4 . Recall that the set $\mathcal{R}_{b}^{S_{0}}$ contains 352 processes. Indeed, it holds that $\left|\mathcal{R}_{\kappa}^{S_{0}}\right| \ll\left|\mathcal{R}_{b}^{S_{0}}\right|$.

\section{Related Work}

An untangling of a concurrent system is a mathematical formalism that can be used to describe and analyze the behavior of that system. In this section, we discuss several other formalisms that address the same problem and point out some of the differences between these formalisms and the one of untangling. 
One can use state space techniques when it comes to the automatic analysis and verification of concurrent systems. Rather than performing analysis directly on a net system, state space techniques explore an induced representation, called a transition system. In a nutshell, a transition system induced by a net system $S$ is a directed graph with all the states reachable from $S$ as its nodes and directed edges that encode possible state transitions, i.e., there is an edge in the graph from state $H$ to state $H^{\prime}$ whenever there is a step in $S$ that leads from $H$ to $H^{\prime}$. Unfortunately, state space techniques suffer from the well-known state space explosion problem. In the worst case, all nodes of the induced transition system must be explored to accomplish the envisioned analysis task. This often turns out to be infeasible due to the large size of the transition system.

An unfolding of a concurrent system is a mathematical structure (often infinite) that explicitly represents causality and concurrency relations on operations of the system, as well as all the points of choice between qualitatively different behaviors of the system. Unfoldings are special acyclic graphs that describe states that are reachable from a net system as combinations of nodes rather than dedicating every node to a single state. In [McM92, McM95], Ken McMillan observed that one can represent all the information contained in an unfolding in its truncated finite initial part, which is often called a finite prefix of the unfolding. If adequate construction algorithms are employed, the size of a finite prefix of the unfolding of a concurrent system is never larger - and in practice is by far smaller - than the size of the transition system induced by the same net system. A classic condition for prefix truncation is completeness, where a prefix induced by a concurrent system is complete if it encodes all the steps (reachable states and possible state transitions) in the system. Completeness of prefixes helps to 'unveil' some of the behavioral properties of concurrent systems by allowing their validation in time that is linear in the size of a given finite complete prefix, e.g., any executability problem [ERV02, EH08] can be solved efficiently using a finite complete prefix of the net system, while keeping other properties sufficiently 'concealed', e.g., the problem of deciding deadlock freedom [MR97] of a net system using a finite complete prefix of this system is known to be NP-complete [McM95]. One can rely on special unfolding truncation criteria in order to unveil other behavioral properties in finite parts of unfoldings. In [EH08], truncation criteria that address executability, repeated executability, livelock, and properties expressible in linear temporal logic, are systematized. The strong coupling of behavioral properties of concurrent systems with different configurations of finite prefixes of their unfoldings is mainly due to implicit dependencies between transition occurrences which stem from unfolding truncations.

Merged processes, which are studied in [KKKV06, KM11, RSK13], are compressed representations of finite prefixes of unfoldings with most of the discussed above advantages and disadvantages of finite prefixes of unfoldings. The compression in merged processes is achieved by addressing such causes of the state space explosion as sequences of choices and unsafeness. Many analysis results that were initially proposed for finite prefixes of unfoldings can be transferred to merged processes.

In [Des00], Jörg Desel suggests using processes of net systems for analysis of concurrent systems. As net systems can induce infinite processes, Desel proposes several termination conditions to limit the size of processes. One option is to use a bound in terms of the number of created events and conditions or in terms of construction time. Unfortunately, this simple method does not guarantee that all the behavior of the net system is explored before triggering the termination condition. As a consequence, any analysis on the set of all - discovered in such a way - processes cannot be considered exhaustive. Alternatively, if one decides to distinguish between internal and external actions of a concurrent system, one can further decide to terminate construction of a process of the corresponding net system if the last appended event describes an occurrence of a transition that leads to a state that does not enable any internal action (this corresponds to a situation when the concurrent system waits for an external trigger in order to progress). Unfortunately, given a net system, it is unknown beforehand whether this termination condition will eventually be fulfilled in the system and, hence, it only fits very specific analysis situations, or must be further constrained to guarantee termination. Finally, Desel proposes to terminate construction of a process if its last appended event corresponds to an occurrence of a transition that leads to a state which was already observed during construction of this process or some other process of the same net system. If the net system is bounded, this condition guarantees termination. The same and stronger termination conditions were previously used for truncating infinite unfoldings of net systems [McM95, ERV02]. In fact, every finite complete prefix of the unfolding of a net system $S$ is a compact representation of a set of all processes of $S$ truncated according to this, or a stronger, termination condition and exhaustively merged based on their isomorphic histories.

Every process in an untangling can be seen as a scenario, i.e., a behavioral requirement that describes interdependencies between actions that can occur in the system. In [Fah10, Rei13], scenarios are proposed as a feature to specify behavior of a system. In this context, our work suggests a technique to decompose a given system into a set of its scenarios that collectively specify all and only the behavior of the system. 


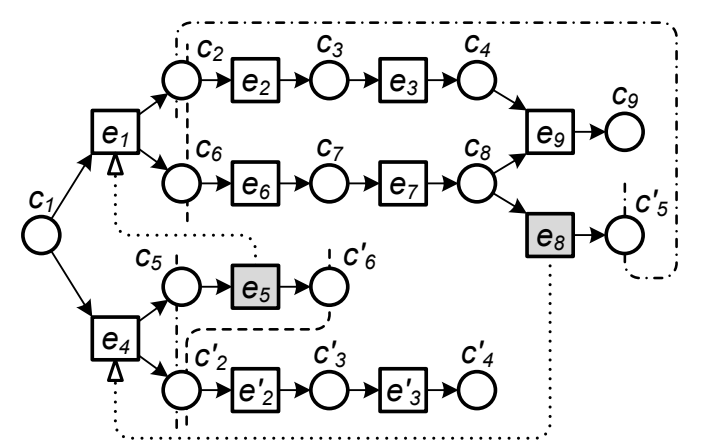

Fig. 10. A finite complete prefix of the unfolding of the net system in Fig. 1(a).

Both unfoldings and untanglings are acyclic graphs that encode causality and concurrency relations on transition occurrences. Thus, fundamentally, untanglings address the state space explosion problem to a similar extent as unfoldings. Furthermore, similar to a finite prefix of the unfolding of a net system $S$, a representative untangling of $S$ is a finite model that describes all the steps in $S$, i.e., all the transition occurrences that are possible from all the states reachable from $S$. However, in contrast to a finite prefix of the unfolding of $S$, a representative untangling of $S$ suggests dedicated scopes for analysis of properties that target individual runs in $S$, i.e., every run in $S$ is represented by some process in a representative untangling of $S$. This result is due to a special termination condition for process construction that limits the size of processes in representative untanglings, cf. Definition 4.3. Several benefits of using representative untanglings for describing concurrent systems are explored in the next section.

\section{Evaluation}

This section ascertains the value of representative untanglings. It illustrates their unique properties, as compared to artifacts studied by the theory of unfoldings, i.e., finite complete prefixes (of unfoldings) of net systems, and demonstrates practical benefits that stem from the use of representative untanglings. The next section contrasts representative untanglings with finite complete prefixes by comparing running times and output artifacts of the reduction-based untangling algorithm (see Section 5.3) and the finite complete prefix algorithm for 1-bounded net systems proposed in [ERV02]. In the subsequent sections, we propose several methods for deciding various behavioral properties of net systems using their representative untanglings and compare computational complexities and running times of these methods with computational complexities and running times of their counterpart techniques that rely on the use of finite complete prefixes. In particular, the behavioral properties addressed are executability, deadlock freedom, and mutual exclusiveness. The results of this evaluation confirm the feasibility and benefits of using representative untanglings in practice.

The evaluation was carried on a real-life collection of 448 net systems (more precisely workflow nets) that model processes from the domains of finance and telecommunication. They were selected from the BIT Library $\left[\mathrm{FFK}^{+} 11\right]$. Net systems that are unsafe were filtering out; a net system is safe iff it is 1-bounded. All the experiments were implemented in Java and performed on a laptop with Intel Core i7-3667U CPU 2.00GHz, 8GB of memory, running Windows 7 and Sun JVM 1.7 (with standard allocation of memory).

\subsection{Size and Construction Time}

In Section 6, we pointed out that representative untanglings have commonalities with finite complete prefixes of unfoldings. Similar to a representative untangling, a finite complete prefix of a net system is a finite acyclic net that encodes the behavior of the corresponding net system. Fig. 10 shows a finite complete prefix of the net system $S$ in Fig. 1(a). This and all the finite complete prefixes constructed in our experiments are generated using the total adequate order for safe systems proposed in [ERV02]. In the prefix, event $e_{5}$ is a cutoff event (highlighted with grey background). Event $e_{5}$ and its corresponding event $e_{1}$ (the relation is denoted by a dotted arrow) are associated with equivalent reachable markings that put one token at place $p_{2}$, one token at place $p_{6}$, and no tokens elsewhere. In the figure, these markings are encoded as sets of conditions 


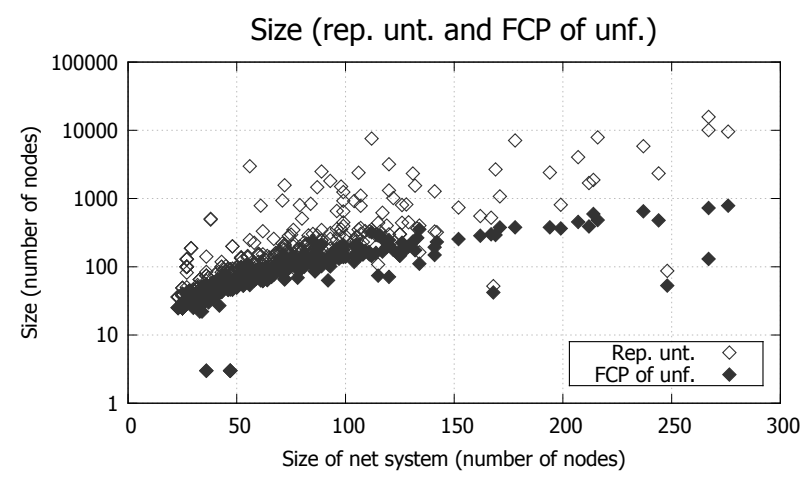

(a)

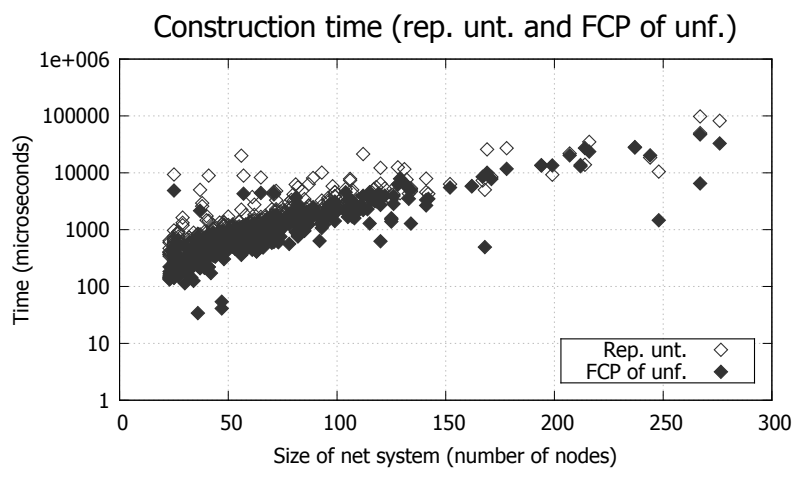

(b)

Fig. 11. Experimental results of computing finite complete prefixes and representative untanglings of 448 net systems taken from practice: (a) number of nodes in constructed artifacts and (b) construction time.

that overlap with dashed lines; refer to [McM95, ERV02] for more information on how events of unfoldings are associated with reachable markings of the corresponding net system. Intuitively, the finite complete prefix in Fig. 10 can be constructed starting from the initial marking of $S$, i.e., from condition $c_{1}$ that encodes the marking with the only token at place $p_{1}$, then iteratively appending events that encode occurrences of transitions (in the order that they can occur in the system), and terminating the construction at cutoff event $e_{5}$ as information on transition occurrences that follow $e_{5}$ is already contained in the part of the prefix that follows its corresponding event $e_{1}$. Similarly, cutoff event $e_{8}$ and its corresponding event $e_{4}$ encode equivalent markings that put one token at place $p_{2}$, one token at place $p_{5}$, and no tokens elsewhere; the respective sets of conditions are denoted by dash-dotted lines in the figure.

Both the baseline and the reduction-based untangling techniques have been implemented and are publicly available as part of the $\mathrm{jBPT}$ initiative [PW13]. ${ }^{10}$ Using this implementation, we conducted an experiment to assess performance of the reduction-based untangling technique in terms of construction times and sizes of the produced outputs. To eliminate load times from the measurements, each experiment was executed six times, and we recorded average times of the second to sixth executions.

Fig. 11 summarizes the results of this test, which can be downloaded and reproduced. ${ }^{11}$ Fig. 11 (a) reports on the sizes (as measured by the number of nodes) of constructed representative untanglings and finite complete prefixes of the 448 net systems. The non-shaded diamonds encode sizes of representative untanglings, while the shaded ones specify sizes of finite complete prefixes. The sizes of net systems from the collection range from 23 to 276 nodes, with the average size being 63.75 nodes. The average size of a finite complete prefix is 91.24 nodes, whereas the average size of a representative untangling is 371.1 nodes. On average, constructed finite complete prefixes and representative untanglings are 1.3 and 3.54 times larger than the corresponding net systems, respectively. The size of a finite complete prefix of a net system is on average $67.39 \%$ of the size of a representative untangling of the same net system. Furthermore, we observed that on average, constructed representative untanglings contain 6.22 processes. Similarly, Fig. 11(b) reports on the times of constructing finite complete prefixes and representative untanglings. According to our tests, the average time spent on constructing a finite complete prefix is 1.57 milliseconds, while the average time spent on constructing a representative untangling is 2.83 milliseconds. ${ }^{12}$

\subsection{Executability}

Finite complete prefixes have proven their advantage when verifying behavioral properties of concurrent systems. For example, given a net system $S$, the executability problem, which consists of deciding whether

\footnotetext{
10 http://code.google.com/p/jbpt

11 http://code.google.com/p/jbpt/wiki/UntanglingsExperiment

12 Uma is used to construct finite complete prefixes of unfoldings: http://service-technology.org/tools/uma
} 
there exists an occurrence sequence in $S$ of which every transition out of a given set of transitions is an element, can be solved in a time that is linear in the size of a finite complete prefix of $S$ [EH08]. More precisely, if a transition $t$ is an element of some occurrence sequence in $S$, then a finite complete prefix of $S$ contains an event that describes an occurrence of $t$. For example, using events of the finite complete prefix in Fig. 10 and the above described rationale, one can trivially conclude that for every transition $t$ of the net system in Fig. 1(a) there exists an occurrence sequence in this net system of which $t$ is an element.

The executability problem is a fundamental problem in concurrency theory as many other problems can be reduced to it, e.g., a solution to the executability problem can help deciding reachability and safety [EH08, GW93]. The executability problem can be formulated as follows.

Definition 7.1 (Executability). A net system $S:=(N, M), N:=(P, T, F)$, can execute some transition in $U \subseteq T$ iff there exist an occurrence sequence $\sigma$ in $S$ and a transition $t \in U$ such that $t$ is an element of $\sigma$.

One can use representative untanglings to solve the executability problem.

Lemma 7.2 (Executability). Let $\Pi$ be a representative untangling of a net system $S:=(N, M), N:=$ $(P, T, F)$. Then, $S$ can execute some transition in $U \subseteq T$ iff there exist a process $\pi \in \Pi, \pi:=\left(N_{\pi}, \rho\right)$, $N_{\pi}:=(B, E, G)$, and an event $e \in E$ such that $\rho(e) \in U$.

Proof. We prove each direction of the statement separately.

$(\Rightarrow)$ Assume that there exists an occurrence sequence that contains $t \in U$ as an element. Then, there exists a run $\delta$ in $S$ that contains a step $\chi:=\left(H, t, H^{\prime}\right)$. According to Definition 4.1 , there exists a process $\pi \in \Pi$, $\pi:=\left(N_{\pi}, \rho\right), N_{\pi}:=(B, E, G)$, that represents every step of $\delta$. Then, $\pi$ represents $\chi$ and, thus, contains an event $e \in E$ such that $\rho(e) \in U$.

$(\Leftarrow)$ Assume that there exist a process $\pi \in \Pi, \pi:=\left(N_{\pi}, \rho\right), N_{\pi}:=(B, E, G)$, and an event $e \in E$ such that $\rho(e) \in U$. According to Lemma 1 in [Des00], there is a run in $S$ which contains a step that describes an occurrence of $\rho(e)$. Thus, there is an occurrence sequence in $S$ which contains $\rho(e)$ as an element.

Therefore, similar to finite complete prefixes, representative untanglings can be employed to solve the executability problem efficiently.

Proposition 7.3 (Executability).

Given a representative untangling $\Pi$ of a net system $S:=(N, M), N:=(P, T, F)$, the following problem can be solved in linear time on $\Pi$ : To decide if $S$ can execute some transition in $U \subseteq T$.

The proof of Proposition 7.3 is a direct consequence of Lemma 7.2. Given a net system $S$ and a subset of its transitions $U$, one can verify whether $S$ can execute some transition in $U$ by visiting events of a representative untangling of $S$ (such that each event is visited at most once) until none of the visited events describes an occurrence of some transition in $U$. The linear time complexity reported in Proposition 7.3 assumes that the set membership operation can be performed in constant time, e.g., as a search in a hash table. For example, using events of the five processes in Figs. 2 and 4, one can decide that the net system $S$ in Fig. 1(a) can execute some transition in every non-empty subset of transitions of $S$; recall that the five processes in Figs. 2 and 4 constitute a representative untangling of $S$.

Fig. 12 reports average times spent when solving executability problems on representative untanglings (rep. unt.) and on finite complete prefixes of unfoldings (FCP of unf.) of the 448 net systems under evaluation. Fig. 12(a), Fig. 12(b), and Fig. 12(c), show the average times spent on solving executability problems on input sets that consist of one, six, and eleven transitions, respectively; note that the smallest net in the collection has eleven transitions. For a given number $n$ of transitions and a given net system $S, n$ transitions of $S$ are randomly selected and the time required for solving the executability problem for these transitions is computed. The average value for this combination of number $n$ and net system $S$ is determined by taking the average of 1000 repetitions of this procedure. Fig. 12(d) shows the average times spent on solving the executability problems for all 448 net systems in the collection for different sizes of input sets of transitions. Again, for a given number $n$ of transitions and a net system $S, n$ transitions of a net system are randomly selected and the time required for solving the executability problem for these transitions is computed. The average value for this number $n$ is obtained by taking the average of 448 values of time spent on executing the above described procedure (one value for every net system in the collection).

Because solutions to the executability problem have linear time complexity, both on representative untanglings and on finite complete prefixes, and considering that representative untanglings are never smaller than finite complete prefixes (refer to Section 7.1), it does not come as a surprise that the times spent 


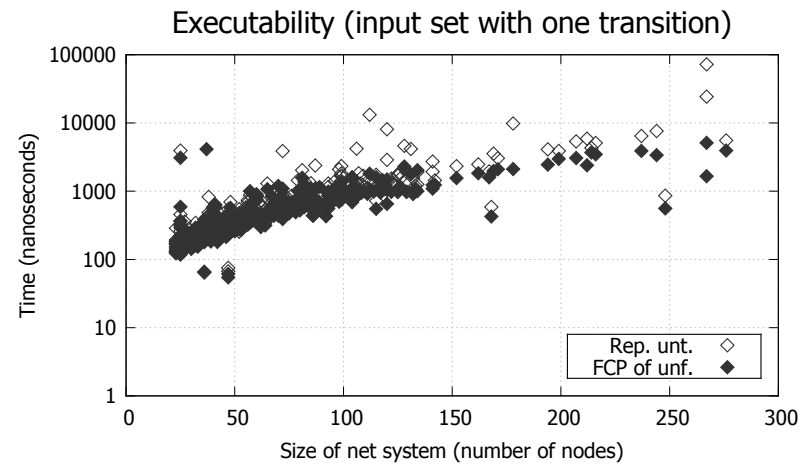

(a)

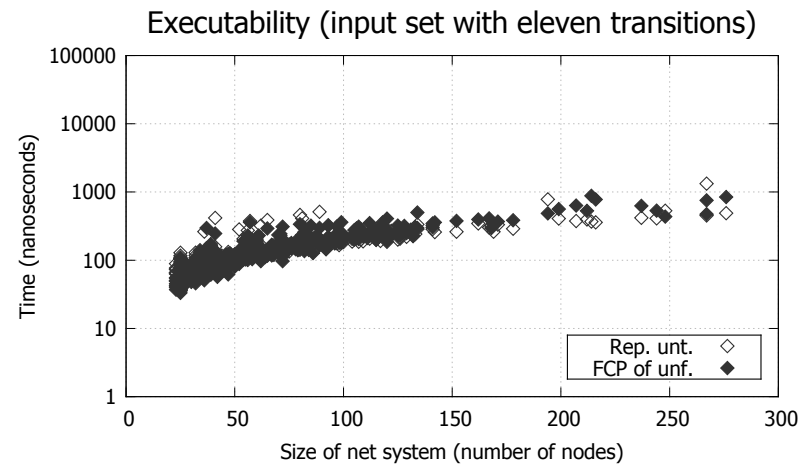

(c)

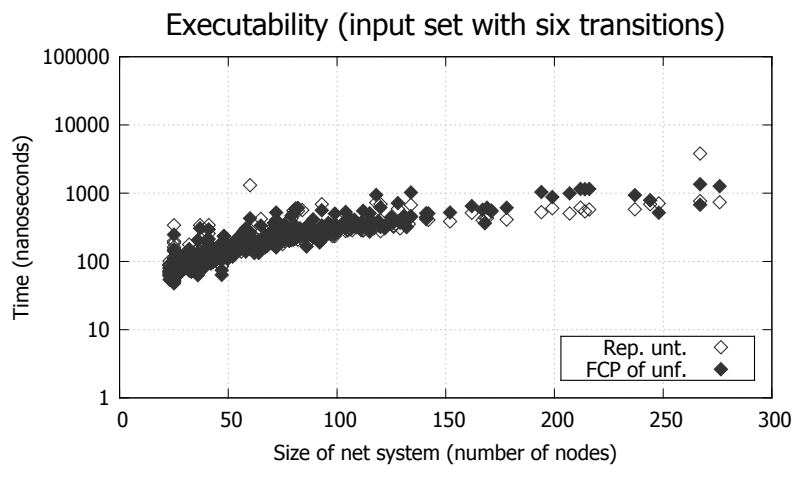

(b)

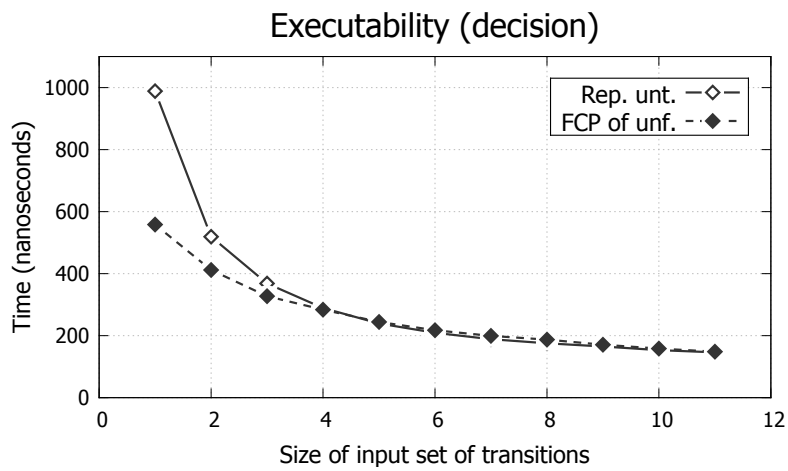

(d)

Fig. 12. Average times of deciding executability using finite complete prefixes and representative untanglings: (a) with an input set that contains one transition, (b) with an input set that contains six transitions, (c) with an input set that contains eleven transitions, and (d) over all net systems in the collection.

on deciding executability based on finite complete prefixes are often smaller than those on representative untanglings. However, the measurements also show that with the growth of the size of input sets of transitions this difference becomes negligible. We observed that certain decisions based on representative untanglings were carried out faster than those based on finite complete prefixes. These are the cases when visited parts of the untanglings turned out to be smaller than visited parts of the respective finite complete prefixes.

Given a net system $S$ and a subset of its transitions $U$, one can check if $S$ can execute some transition in $U$ by verifying whether $U$ overlaps with the set of all transitions that are elements of all occurrence sequences in $S$. Clearly, this set consists only of those transitions that are described by at least one event of a representative untangling of $S$ or, similarly, by at least one event of a finite complete prefix of $S$. For the net systems under evaluation, on average such sets were constructed in 18 and 33 microseconds using finite complete prefixes and representative untanglings, respectively.

The performed tests hint at the fact that differences between running times of techniques for solving decision problems of the same time complexity which operate on representative untanglings and finite complete prefixes can often be negligible in practice. Further experiments are required to justify this claim.

In [PLtH14], we introduced the total executability problem. Given a net system $S$ and a subset of its transitions $U$, the total executability problem consists of deciding whether there exists an occurrence sequence in $S$ of which every transition in $U$ is an element. We showed that this problem can be solved efficiently using a representative untangling of $S$ and hinted at the fact that the proposed solution can be of help when designing automated retrieval systems that query for behavioral information in collections of process models.

\subsection{Deadlock freedom}

Another important problem in concurrency theory is the deadlock freedom problem. It deals with answering the question whether there exists a deadlock marking reachable from a given net system. 


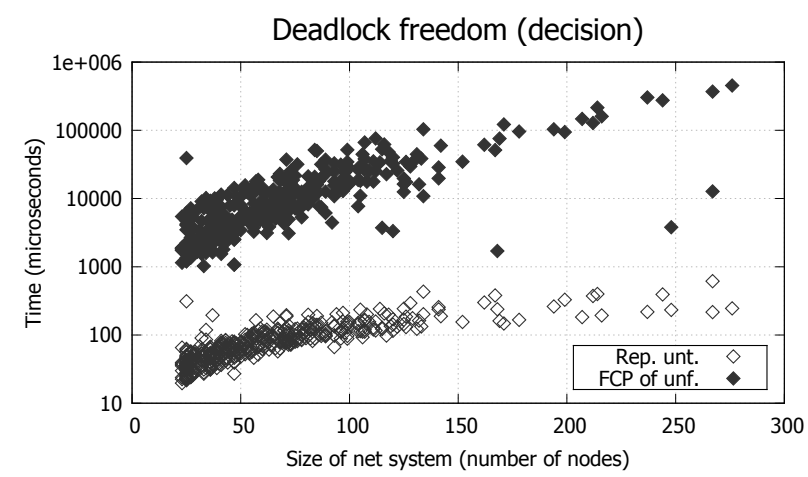

(a)

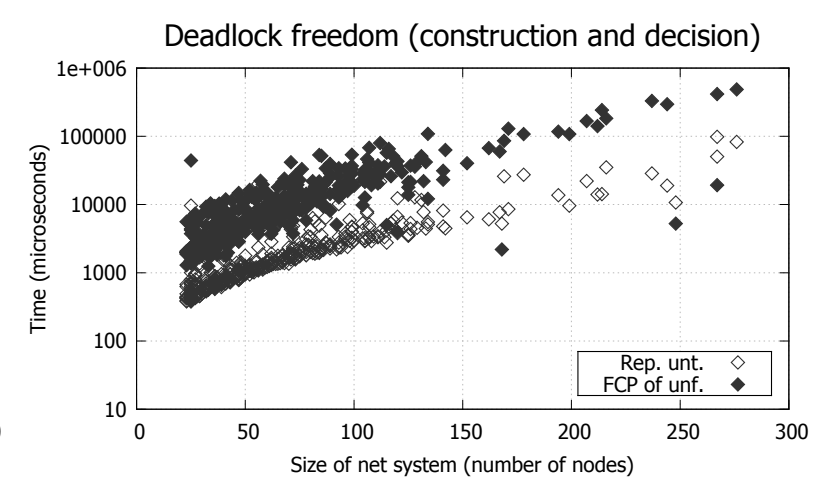

(b)

Fig. 13. Average times of deciding deadlock freedom using finite complete prefixes and representative untanglings: (a) decision times and (b) times spent to construct auxiliary artifacts plus decision times.

Definition 7.4 (Deadlock freedom). A marking $M$ of a net $N:=(P, T, F)$ is a deadlock marking iff there exists no transition $t \in T$ that is enabled in $(N, M)$. A net system $S:=(N, M)$ is deadlock free iff there exists no deadlock marking of $N$ reachable from $S$.

The problem of deciding deadlock freedom of a bounded net system $S$ is known to be NP-complete on a finite complete prefix of the unfolding of $S$ [McM95, MR97]. Alternatively, one can use a representative untangling of $S$ to decide deadlock freedom.

Lemma 7.5 (Deadlock freedom).

Let $\Pi$ be a representative untangling of a net system $S:=(N, M) . S$ is deadlock free iff for every process $\pi:=\left(N_{\pi}, \rho\right)$ in $\Pi$ it holds that $\left(C,\left.\rho\right|_{C}\right)$, where $C:=\operatorname{Max}\left(N_{\pi}\right)$, is not a deadlock marking of $N$.

Proof. We prove each direction of the statement separately.

$(\Rightarrow)$ If $S$ is deadlock free then every marking that is reachable from $S$ is not a deadlock marking of $N$.

Because every maximal cut of every process in $\Pi$ corresponds to some marking that is reachable from $S$, refer to Theorem 2.9 for details, it trivially holds that all maximal cuts of all processes in $\Pi$ do not correspond to deadlock markings.

$(\Leftarrow)$ Assume that for every process $\pi:=\left(N_{\pi}, \rho\right)$ in $\Pi$ it holds that $\left(C,\left.\rho\right|_{C}\right)$, where $C:=\operatorname{Max}\left(N_{\pi}\right)$, is not a deadlock marking of $N$, but $S$ is not deadlock free. Then, there exists a deadlock marking $M^{\prime}$ of $N$ reachable from $S$ via some run $\delta$ in $S$. There also exists a process $\pi:=\left(N_{\pi}, \rho\right)$ in $\Pi$ that represents $\delta$, $N_{\pi}:=(B, E, G)$. Because $\pi$ represents every step of $\delta$, it holds that there exists a cut $C^{\prime}$ of $N_{\pi}$ that corresponds to marking $M^{\prime}$. If $C^{\prime}$ is not the maximal cut of $N_{\pi}$, then $M^{\prime}$ is not a deadlock marking of $N$ ( $M^{\prime}$ enables some transition $\rho(e)$, where $e \in E$ and $\bullet e \subseteq C^{\prime}$ ). Then, it holds that $C^{\prime}$ is the maximal cut of $N_{\pi}$, which leads to a contradiction.

It is easy to see that deadlock freedom of a net system $S$ can be decided efficiently on a representative untangling of $S$.

Proposition 7.6 (Deadlock freedom). Given a representative untangling $\Pi$ of a net system $S$, the following problem can be solved in linear time on $\Pi$ : To decide if $S$ is deadlock free.

The proof of Proposition 7.6 is a direct consequence of Lemma 7.5. Indeed, to check deadlock freedom of $S$ it suffices to test if the maximal cut of some process of a representative untangling of $S$ induces a deadlock marking. This check can be accomplished during a single scan through all conditions of the representative untangling. For example, one can decide that the net system $S$ in Fig. 1(a) is not deadlock free based on the maximal cut $D_{3}:=\left\{c_{9}\right\}$ of the causal net in Fig. 4(a). This cut induces a deadlock marking of the net in Fig. 1(a) which puts one token at place $p_{9}$ and no tokens elsewhere; recall that the process in Fig. 4(a) is one out of the five processes shown in Figs. 2 and 4, which together constitute a representative untangling of $S$.

Fig. 13 reports average times (on a logarithmic scale) of deciding deadlock freedom for each of the 448 net systems under evaluation based on representative untanglings (rep. unt.) and finite complete prefixes of 
unfoldings (FCP of unf.). The checks on finite complete prefixes follow the linear algebraic approach proposed in [MR97]. The implementation relies on LpSolve ver. 5.5.2.0 when solving systems of inequalities. ${ }^{13}$ Fig. $13(\mathrm{a})$ suggests the times spent to carry out the decisions considering that representative untanglings and finite complete prefixes are already constructed, whereas Fig. 13(b) shows overall times spent to decide deadlock freedom, i.e., the times spent on constructing auxiliary artifacts (either representative untanglings or finite complete prefixes) plus the times spent to carry out the decisions based on the subsequent checks on the respective artifacts. To eliminate load times from the measurements, each deadlock freedom problem was solved six times, and we recorded average times spent on the second to sixth problem solving exercise. On average, it took 89.99 microseconds and 15.66 milliseconds to decide deadlock freedom based on representative untanglings and finite complete prefixes, respectively. When taking the times spent on constructing the auxiliary artifacts into account, the average times of checking deadlock freedom increase to 2.92 and 17.23 milliseconds, respectively.

In most of the cases, the checks based on representative untanglings outperform by far the checks that rely on the use of finite complete prefixes. The significant difference between decision times on representative untanglings and finite complete prefixes, refer to Fig. 13(a), confirms that most of the value when using representative untanglings comes from their reuse for checking multiple behavioral properties, e.g., once a representative untangling of a net system is constructed it can be used to decide deadlock freedom but also to solve an arbitrary number of instances of the executability problem.

\subsection{Mutual Exclusiveness}

Together with the studies of fundamental problems in concurrency theory, such as the executability problem and the deadlock freedom problem, much of attention of researchers has been devoted to decidability issues for specification languages in which a large number of behavioral properties can be expressed. In particular, given a model of a system, model checking studies the problem of automatically checking whether the model satisfies a behavioral property specified in a language that often takes the form of a temporal logic. Linear time logics [Pnu77] for Petri nets form a class of temporal logics that are usually interpreted on maximal computations. One behavioral phenomenon that can be conveniently expressed in terms of a linear time logic is mutual exclusiveness of events, or the conflict relation, which is one of the fundamental behavioral relations as classified by the $4 \mathrm{C}$ spectrum $\left[\mathrm{PWC}^{+} 14\right]$. In general, two events are mutually exclusive if they cannot occur together. For instance, two events of observing heads or tails when tossing a coin are mutually exclusive. The phenomenon of mutual exclusiveness of events can be adapted to events that describe occurrences of transitions and can consequently be characterized on transitions of Petri nets as follows.

Definition 7.7 (Mutual exclusiveness).

Let $S:=(N, M), N:=(P, T, F)$, be a net system. Transitions $t_{1} \in T$ and $t_{2} \in T$ are mutually exclusive in $S$ iff for every occurrence sequence $\sigma$ in $S$ it holds that either $t_{1}$ or $t_{2}$ is not an element of $\sigma$.

For a transition $t \in T$ it trivially holds that $t$ is mutually exclusive with itself if and only if $t$ is not an element of any occurrence sequence in $S$. Clearly, mutual exclusiveness is a symmetric relation.

One can rely on finite complete prefixes when model checking behavioral properties of corresponding net systems, e.g., mutual exclusiveness of transitions. Given a net system $S$ and a linear temporal logic formula, a common approach is to augment $S$ with a Büchi tester [EH08] (a new component added to $S$ ) in order to observe and register occurrences of certain transitions in a finite complete prefix of the unfolding of the augmented net system. These transition occurrences signify violations of the property specified in the formula. Thus, when model checking mutual exclusiveness of two transitions of a given net system a fresh finite complete prefix of an augmented version of the system must be constructed. For instance, if one wants to check mutual exclusiveness of every distinct pair of transitions in the net system in Fig. 1(a), one needs to construct and analyze 36 finite complete prefixes! Additionally, one finite complete prefix must be constructed to check whether each transition of the system is related with itself via mutual exclusiveness. Note that one can check whether a transition is an element of some occurrence sequence in a net system by using the solution to the executability problem proposed in [EH08].

In contrast, one can rely on a single representative untangling of a net system to efficiently decide mutual exclusiveness for all pairs of transitions of this net system.

\footnotetext{
$\overline{13 \text { http://lpsolve.sourceforge.net/ }}$
} 
Lemma 7.8 (Mutual exclusiveness - untanglings).

Let $\Pi$ be a representative untangling of a net system $S:=(N, M), N:=(P, T, F)$. Transitions $t_{1} \in T$ and $t_{2} \in T$ are mutually exclusive in $S$ iff for every process $\pi \in \Pi, \pi:=\left(N_{\pi}, \rho\right), N_{\pi}:=(B, E, G)$, and for every two events $e_{1} \in E$ and $e_{2} \in E$ it holds that either $\rho\left(e_{1}\right) \neq t_{1}$ or $\rho\left(e_{2}\right) \neq t_{2}$

Proof. We prove each direction of the statement separately.

$(\Rightarrow)$ Assume that for every occurrence sequence $\sigma$ in $S$ it holds that either $t_{1}$ or $t_{2}$ is not an element of $\sigma$, but there exist a process $\pi \in \Pi, \pi:=\left(N_{\pi}, \rho\right), N_{\pi}:=(B, E, G)$, and events $e_{1}, e_{2} \in E$ such that $\rho\left(e_{1}\right)=t_{1}$ and $\rho\left(e_{2}\right)=t_{2}$. Then, according to Lemma 1 in [Des00], there exists an occurrence sequence in $S$ of which both $t_{1}$ and $t_{2}$ are elements.

$(\Leftarrow)$ Assume that for every process $\pi \in \Pi, \pi:=\left(N_{\pi}, \rho\right), N_{\pi}:=(B, E, G)$, and for every two events $e_{1} \in E$ and $e_{2} \in E$ it holds that either $\rho\left(e_{1}\right) \neq t_{1}$ or $\rho\left(e_{2}\right) \neq t_{2}$. According to Definition 4.1, every run in $S$ is represented by some process $\pi \in \Pi$. Then, there exists no run and, thus, no occurrence sequence $\sigma$ in $S$ such that $t_{1}$ and $t_{2}$ are elements of $\sigma$.

Given a net system $S$, one can efficiently decide whether two transitions of $S$ are mutually exclusive using a representative untangling of $S$.

Proposition 7.9 (Mutual exclusiveness).

Given a representative untangling $\Pi$ of a net system $S:=(N, M), N:=(P, T, F)$, the following problem can be solved in linear time on $\Pi$ : To decide if transitions $t_{1} \in T$ and $t_{2} \in T$ are mutually exclusive in $S$.

The proof of Proposition 7.9 is a direct consequence of Lemma 7.8. Indeed, given two transitions $t_{1}$ and $t_{2}$ of a net system $S$, one can verify whether they are mutually exclusive in $S$ by checking if a representative untangling of $S$ contains a process with events that describe occurrences of $t_{1}$ and $t_{2}$. For example, one can use the processes in Figs. 2 and 4 to verify that transitions $t_{1}$ and $t_{4}$ are the only two mutually exclusive transitions in the net system in Fig. 1(a). Indeed, none of the five processes in the two figures contains an event that describes an occurrence of transition $t_{1}$ and an event that describes an occurrence of transition $t_{4}$.

Next, we compare two techniques for computing the mutual exclusiveness relation on transitions of a net system, one using a representative untangling of the net system and the other relying on the study of a finite complete prefix of the net system. To have a fair comparison, instead of adopting the model checking approach proposed in [EH08], which as discussed above requires us to construct a fresh finite complete prefix for each pair of distinct transitions of the net system, we reduce the problem to the covering problem [Rac78], which can be solved for all pairs of transitions on a single finite complete prefix of a specially augmented version of the net system. More precisely, prior to constructing a finite complete prefix of a net system, it gets transformed via injections of guards. An injection of a guard for a transition of a net system results in a structural transformation of the net system. This structural transformation was used in $\left[\mathrm{PWC}^{+} 14\right]$ to reduce the problem of computing can conflict and can co-occur relations of the $4 \mathrm{C}$ spectrum to the reachability problem [Hac75], refer to Definition 5.1 and Proposition 5.2 in $\left[\mathrm{PWC}^{+} 14\right]$. Finally, given a net system $S:=\left(N, M_{0}\right), N:=(P, T, F)$, and a marking $M:=(K, \mu)$, the covering problem consists of deciding whether there exists a marking $M^{\prime}:=\left(K^{\prime}, \mu^{\prime}\right)$ that is reachable from $S$ and covers $M$, where $M^{\prime}$ covers $M$ iff for every place $p \in P$ it holds that $\mu^{\prime}$ assigns at least as many tokens to $p$ as $\mu$.

Fig. 14(a) shows the net system $S^{\prime}$ obtained from the net system $S$ in Fig. 1(a) via injections of guards for transitions $t_{4}$ and $t_{8}$. In the figure, fresh places and transitions added to $S$ are highlighted with grey background. Transitions $\hat{t}_{4}$ and $\hat{t}_{8}$ are the guard transitions for $t_{4}$ and $t_{8}$, respectively. Places $q$ and $s$ are the control places for $t_{4}$ and $t_{8}$, respectively. A guard transition $t^{\prime}$ for a transition $t$ can occur instead of $t$ and implements the effect of an occurrence of $t$, i.e., the preset and postset of $t^{\prime}$ contains the preset and postset of $t$, respectively. For every run $\delta$ in $S^{\prime}$ it holds that $t^{\prime}$ can occur at most once in $\delta$. This is ensured by one fresh token assigned to the special fresh input place of $t^{\prime}$, e.g., see places $p$ and $r$ in Fig. 14(a). An occurrence of $t^{\prime}$ 'allows' subsequent occurrences of $t$ by producing a token at the control place for $t$.

Clearly, different orderings of injections of guards in $S$ for all transitions from a given set of transitions of $S$ lead to the same resulting net system $S^{\prime}$. Moreover, occurrence sequences in $S$ and $S^{\prime}$ are closely related. In particular, one can construct an occurrence sequence in $S$ from an occurrence sequence $\delta$ in $S^{\prime}$ by replacing every occurrence of every guard transition in $\delta$ with an occurrence of a transition that this guard was introduced for. Similarly, one can construct an occurrence sequence in $S^{\prime}$ from an occurrence sequence $\delta$ in $S$ by replacing all first occurrences of transitions for which guard transitions in $S$ were introduced with the respective guard transitions. For example, $t_{4} t_{5} t_{6} t_{7} t_{8} t_{5} t_{6} t_{7} t_{8}$ and $\hat{t}_{4} t_{5} t_{6} t_{7} \hat{t}_{8} t_{5} t_{6} t_{7} t_{8}$ are two such related occurrence sequences in the net systems in Fig. 1(a) and Fig. 14(a), respectively. 


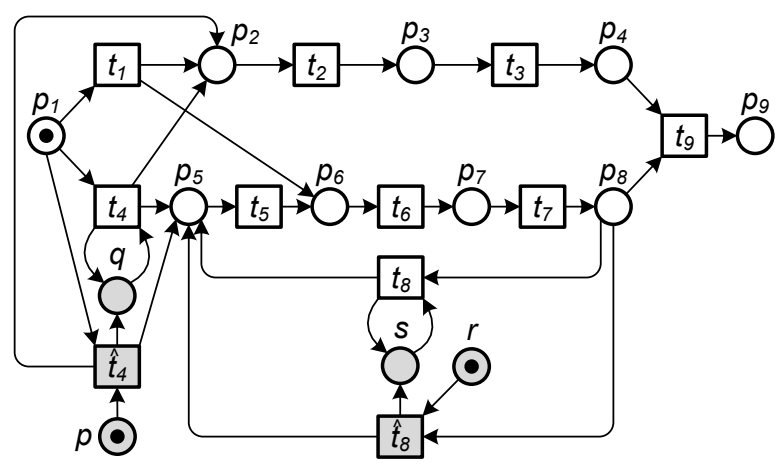

(a)

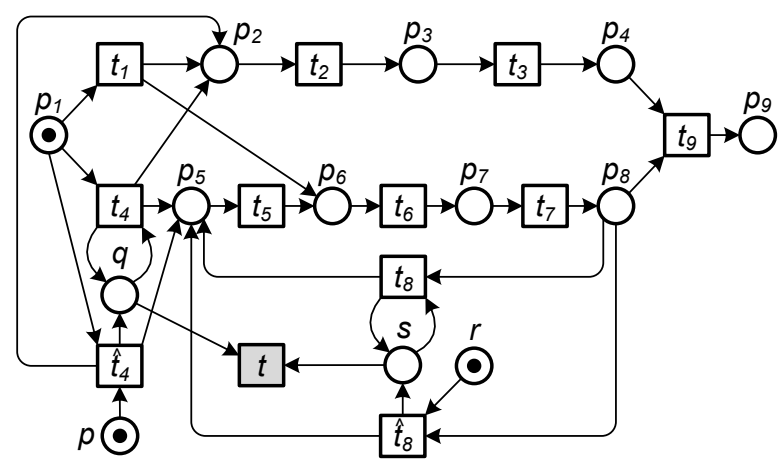

(b)

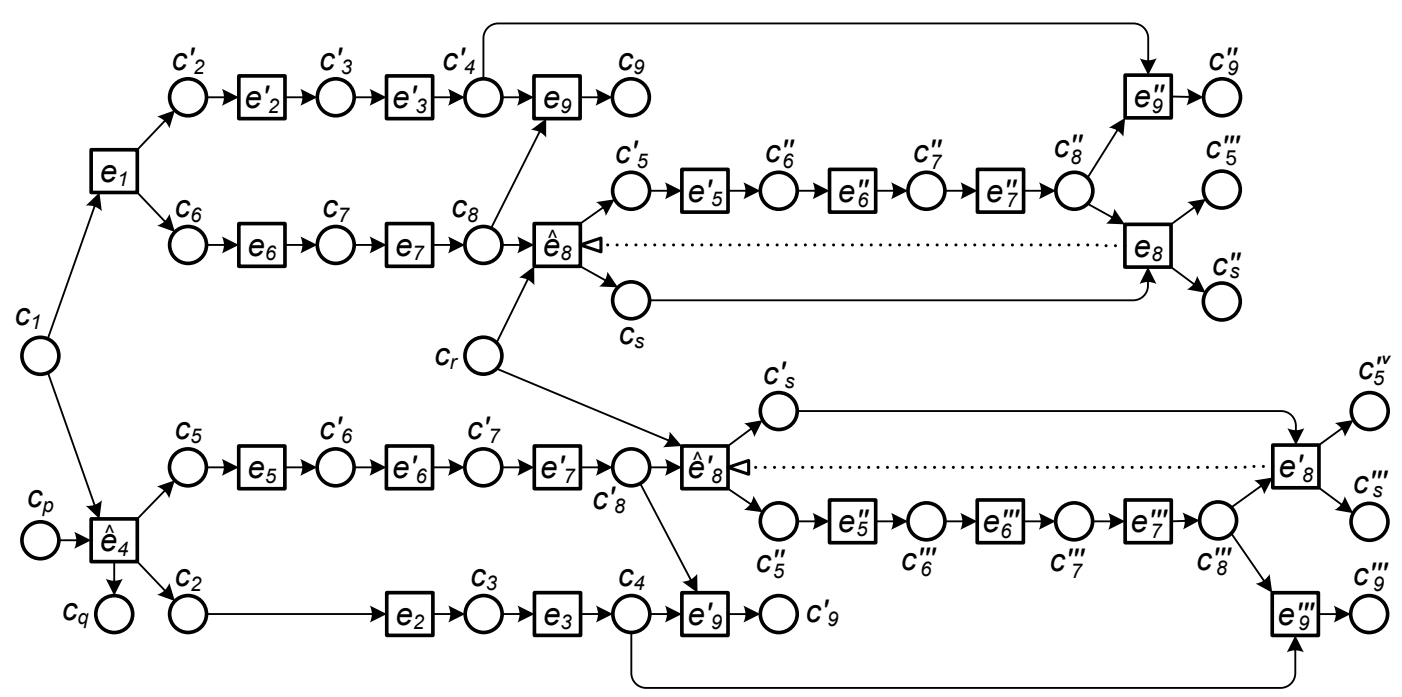

(c)

Fig. 14. (a) A net system obtained from the net system in Fig. 1(a) via injections of guards for transitions $t_{4}$ and $t_{8}$, (b) a net system obtained from the net system in (a) by adding control transition $t$ for places $q$ and $s$, and (c) a finite complete prefix of the net system in (a).

Lemma 7.10 (Mutual exclusiveness - the covering problem).

Let $S:=(N, M), N:=(P, T, F)$, be a net system and let $S^{\prime}:=\left(N^{\prime}, M^{\prime}\right), N^{\prime}:=\left(P^{\prime}, T^{\prime}, F^{\prime}\right)$, be a net system obtained via injections of guards in $S$, cf. Definition 5.1 in $\left[\mathrm{PWC}^{+} 14\right]$, for all transitions in $T$. Let $t_{1} \in T$ and $t_{2} \in T$ be transitions of $N$, where $p_{1} \in P^{\prime}$ and $p_{2} \in P^{\prime}$ are the control places for $t_{1}$ and $t_{2}$, respectively. Transitions $t_{1}$ and $t_{2}$ are mutually exclusive in $S$ iff for every marking $M^{\prime \prime}$ that is reachable from $S^{\prime}$ it holds that $M^{\prime \prime}$ does not cover a marking of $N^{\prime}$ that induces the state $\left[p_{1} p_{2}\right]$.

Indeed, if $t_{1}$ and $t_{2}$ are mutually exclusive then there exists no occurrence sequence in $S$ of which $t_{1}$ and $t_{2}$ are elements. Hence, there exists no occurrence sequence in $S^{\prime}$ of which the guard transitions for $t_{1}$ and $t_{2}$ are elements. Then, one cannot reach a marking from $S^{\prime}$ that covers a marking that puts one token at place $p_{1}$, one token at place $p_{2}$, and no tokens elsewhere. Moreover, if one cannot reach a marking from $S^{\prime}$ that assigns tokens to $p_{1}$ and $p_{2}$ then there exists no occurrence sequence in $S^{\prime}$ that contains occurrences of the respective guard transitions. Consequently, one cannot find an occurrence sequence in $S^{\prime}$ from which it is possible to construct an occurrence sequence in $S$ of which both $t_{1}$ and $t_{2}$ are elements.

Note that the covering problem is decidable [Rac78]. The procedure for deciding the covering problem proposed in [Rac78] operates in space exponential in the size of the input net system. Alternatively, one can 


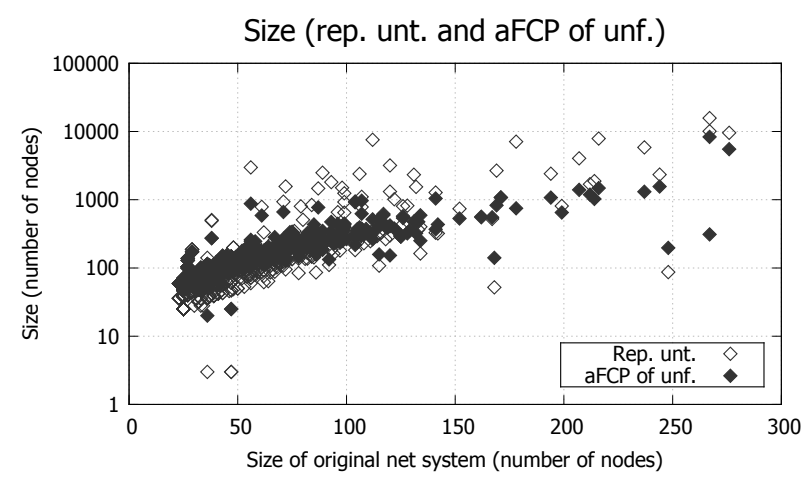

(a)

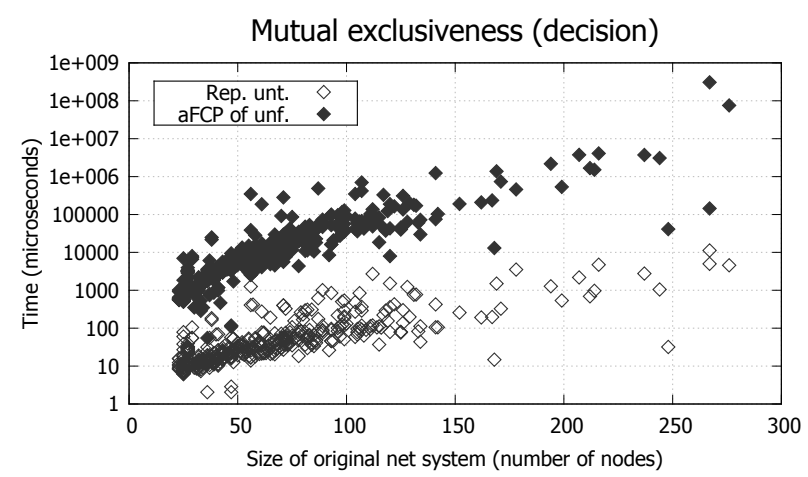

(c)

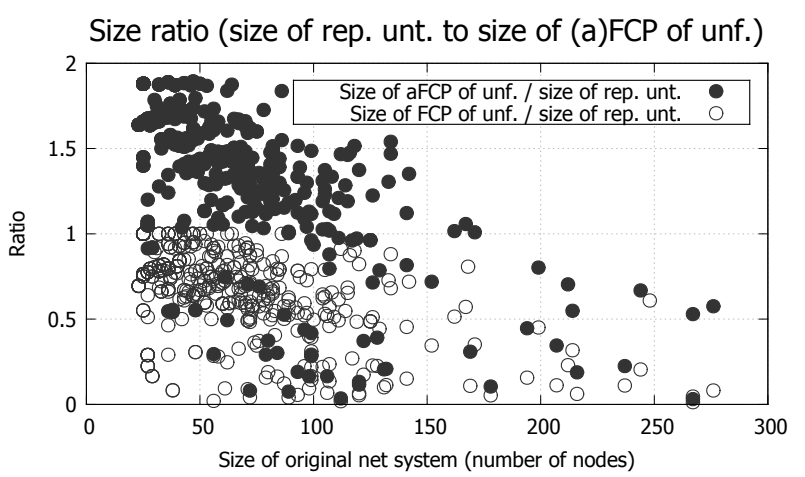

(b)

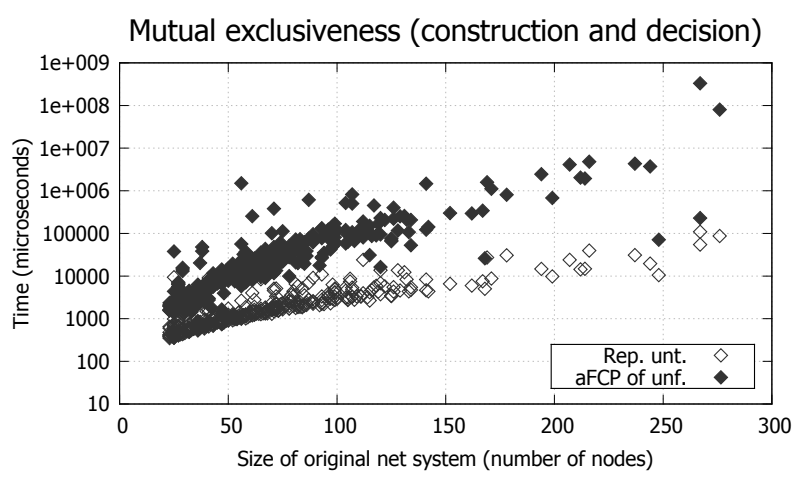

(d)

Fig. 15. (a) Sizes of representative untanglings of the original net systems and of finite complete prefixes of the augmented net systems obtained after injecting guards for all transitions, (b) ratios between sizes of finite complete prefixes and representative untanglings of original and augmented net systems, and average times of deciding mutual exclusiveness on all transitions of net systems using representative untanglings and finite complete prefixes: (c) decision times and (d) times spent on constructing auxiliary artifacts plus decision times.

use finite complete prefixes when deciding the covering problem. The procedures for deciding the covering problem of net systems on their finite complete prefixes operate in times that are exponential in the sizes of the prefixes [Kho03]. However, these times are often acceptable in practice.

We performed a number of measurements to compare two techniques for computing the mutual exclusiveness relation on transitions of net systems in the collection under evaluation, one that uses representative untanglings (as per Lemma 7.8) and the other that relies on the solution to the covering problem on finite complete prefixes (as per Lemma 7.10), cf. [Kho03] for details.

Fig. 15(a) reports on sizes (as measured by the number of nodes) of constructed representative untanglings (rep. unt.) of the original net systems (these sizes are the same as those reported in Fig. 11(a)) and finite complete prefixes of the augmented net systems obtained after injections of guards for all transitions (aFCP of unf.); again, note the use of a logarithmic scale. Observe that injections of guards preserve safeness of systems, which allows us to keep using the total adequate order for safe systems proposed in [ERV02] when constructing the prefixes. Similar to Fig. 11(a), the non-shaded diamonds encode sizes of representative untanglings, whereas the shaded ones give sizes of finite complete prefixes.

Though construction of a finite complete prefix of a bounded net system is considered to be efficient in practice, it is of an exponential nature and can easily explode [KKKV06], both in terms of computation time and the size of the resulting prefix. For instance, Fig. 14(c) shows a finite complete prefix of the net system in Fig. 14(a); again the dotted arrows encode relations between cutoffs and corresponding events of the prefix. The prefix is composed of 60 nodes (conditions and events). Note that the finite complete prefix of the original net system (before injections of guards) is shown in Fig. 10 and consists of 25 nodes. 
For every net system $S$ in the collection, Fig. 15(b) reports on the ratio of the size of the finite complete prefix of $S$ to the size of the representative untangling of $S$. The non-shaded circles encode ratios of sizes of finite complete prefixes of original net systems (FCP of unf.) to sizes of their representative untanglings (rep. unt.). For every constructed representative untangling, it holds that it is either of the same size or is larger than the constructed finite complete prefix of the corresponding net system, i.e., the respective ratio is less than or equal to one. The situation changes once net systems get augmented via injections of guards (a guard is injected for every transition of every system in the collection). The shaded circles in Fig. 15(b) report on ratios of sizes of finite complete prefixes of augmented net systems (aFCP of unf.) to sizes of representative untanglings of original net systems (rep. unt.). In most of the cases finite complete prefixes turn to be larger in size than their respective representative untanglings. On average, we observed that the size of the constructed representative untangling of the original net system is $70 \%$ of the size of the constructed finite complete prefix of the corresponding augmented net system.

Fig. 15(c) reports average times (on a logarithmic scale) of computing the mutual exclusiveness relations on transitions for the 448 net systems under evaluation using representative untanglings (as per Lemma 7.8) and finite complete prefixes of unfoldings of augmented net systems (as per Lemma 7.10). On average, a mutual exclusiveness relation was computed in 169 microseconds and 931 milliseconds using representative untanglings (rep. unt.) and finite complete prefixes (aFCP of unf.), respectively. Similarly, Fig. 15(d) reports overall times spent on constructing auxiliary artifacts, either representative untanglings or finite complete prefixes of augmented net systems, and subsequently computing the mutual exclusiveness relation using the respective artifacts. On average, the observed overall time of computing the mutual exclusiveness relation for a net system $S$, i.e., deciding mutual exclusiveness for every pair of transitions of $S$, is 3 and 1027 milliseconds using its representative untangling and the finite complete prefix of its augmented version, respectively.

In [McM92], Ken McMillan proposes an alternative method for solving the covering problem for a safe net system, which relies on the use of a finite complete prefix of the system. If one wants to verify whether a given set of places of a net system can ever be marked simultaneously at some of its reachable markings, one first needs to add a fresh control transition with the given places as inputs and without output places; note that in order to use this method we need to violate the requirement of every net being T-restricted. Consequently, if a finite complete prefix of the resulting net system contains an event that describes an occurrence of the control transition, then from the original net system one can reach a marking that covers a marking that puts one token at every place in the given set of places and no tokens at the other places. Hence, one can organize the computation of the mutual exclusiveness relation by augmenting a net system obtained after injections of guards in a given net system further by adding one fresh transition for every two distinct injected control places, such that these two places are its only inputs. Then, one can decide mutual exclusiveness for every two distinct transitions of the given net system by registering events that describe occurrences of the control transitions during a single traversal over all the events in a finite complete prefix of the resulting net system. For example, Fig. 14(b) shows the net system obtained from the net system in Fig. 14(a) by adding control transition $t$ (highlighted with grey background) for the control places $q$ and $s$. One can decide mutual exclusiveness of transitions $t_{4}$ and $t_{8}$ in the net system in Fig. 1(a) based on the presence of events that describe occurrences of $t$ in a finite complete prefix of the unfolding of the net system in Fig. 14(b). Though the described procedure is efficient, finite complete prefixes of net systems after injections of guards and subsequent insertions of control transitions tend to explode in size. For instance, the finite complete prefix of net system $S^{\prime}$ obtained from net system $S$ in Fig. 1(a) after injecting guards for all transitions of $S$ is composed of 90 nodes, whereas the finite complete prefix of net system $S^{\prime \prime}$ obtained from $S^{\prime}$ by adding control transitions for each pair of distinct control places is composed of 193 nodes. For the collection of 448 net systems under evaluation, we observed that the size of the constructed representative untangling of a net system is on average $24 \%$ of the size of the constructed finite complete prefix of the augmented version of the net system obtained after injections of guards and control transitions.

\section{Conclusion}

This article contributes the theory of untanglings, which provides a novel characterization of behaviors encoded in concurrent systems. The theory revolves around the notion of a representative untangling, i.e., an alternative representation of a concurrent system, which strikes a good balance between the size of the model of a system and the time required for analysis of its behavioral properties.

The article proposes a baseline algorithm for constructing representative untanglings of concurrent 
systems. As the name suggests, this algorithm should be used as a reference that specifies basic principles for constructing representative untanglings. The article also contributes a generic framework for improving the baseline algorithm based on structural transformations of input systems, and describes two reduction rules that fit this framework. It is shown that the desired properties of representative untanglings are preserved by this framework. Moreover, an experiment with a real-life collection of concurrent systems confirms the scalability of the improved algorithm both in terms of time and space. Finally, this article demonstrates the use of representative untanglings for analysis of concurrent systems. It is shown that representative untanglings offer significant benefits over the state-of-the-art when deciding behavioral properties of concurrent systems.

Future work can be performed (i) to extend the repertoire of structural transformations that fit the proposed framework for improving the baseline untangling algorithm, (ii) to improve the baseline untangling algorithm, e.g., by introducing mechanisms for pruning redundant (parts of) discovered maximal significant runs, (iii) to introduce the notion of a canonical representative untangling of a system, i.e., a representative untangling of the system with the least number of nodes, and (iv) to propose new analysis techniques that rely on the use of (representative) untanglings.

Acknowledgments This work is partly funded by the Australian Research Council grant LP110100252. NICTA is funded by the Australian Government (Department of Broadband, Communications and the Digital Economy) and the Australian Research Council through the ICT Centre of Excellence program.

\section{References}

[BD90] Eike Best and Jörg Desel. Partial order behaviour and structure of Petri nets. Formal Aspects of Computing (FAC), 2(2):123-138, 1990

[DE95] Jörg Desel and Javier Esparza. Free Choice Petri Nets. Cambridge Tracts in Theoretical Computer Science 40. Cambridge University Press, 1995.

[Des00] Jörg Desel. Validation of process models by construction of process nets. In Business Process Management (BPM), volume 1806 of $L N C S$, pages 110-128. Springer Berlin Heidelberg, 2000.

[EH08] Javier Esparza and Keijo Heljanko. Unfoldings: A Partial-Order Approach to Model Checking. Monographs in Theoretical Computer Science. An EATCS Series. Springer, 2008.

[ERV02] Javier Esparza, Stefan Römer, and Walter Vogler. An improvement of McMillan's unfolding algorithm. Formal Methods in System Design (FMSD), 20(3):285-310, 2002.

[Fah10] Dirk Fahland. From Scenarios to Components. PhD thesis, Humboldt-Universität zu Berlin, Berlin, Germany, 2010.

$\left[\mathrm{FFK}^{+} 11\right]$ Dirk Fahland, Cédric Favre, Jana Koehler, Niels Lohmann, Hagen Völzer, and Karsten Wolf. Analysis on demand: Instantaneous soundness checking of industrial business process models. Data \& Knowledge Engineering (DKE), $70(5): 448-466,2011$.

[GR83] Ursula Goltz and Wolfgang Reisig. The non-sequential behavior of Petri nets. Information and Control (IANDC), 57(2/3):125-147, 1983 .

[GW93] Patrice Godefroid and Pierre Wolper. Using partial orders for the efficient verification of deadlock freedom and safety properties. Formal Methods in System Design (FMSD), 2(2):149-164, 1993.

[Hac75] Michel Hack. Decidability Questions for Petri Nets. Outstanding Dissertations in the Computer Sciences. Garland Publishing, New York, 1975.

[Kho03] Victor Khomenko. Model Checking Based on Prefixes of Petri Net Unfoldings. PhD thesis, University of Newcastle upon Tyne, School of Computing Science, Newcastle upon Tyne, United Kingdom, February 2003.

[KKKV06] Victor Khomenko, Alex Kondratyev, Maciej Koutny, and Walter Vogler. Merged processes: A new condensed representation of Petri net behaviour. Acta Informatica (ACTA), 43(5):307-330, 2006.

[KM11] Victor Khomenko and Andrey Mokhov. An algorithm for direct construction of complete merged processes. In Petri Nets, volume 6709 of LNCS, pages 89-108. Springer Berlin Heidelberg, 2011.

[LSV98] Edward A. Lee and Alberto L. Sangiovanni-Vincentelli. A framework for comparing models of computation. IEEE Transactions on Computer-Aided Design of Integrated Circuits and Systems (TCAD), 17(12):1217-1229, 1998.

[McM92] Kenneth L. McMillan. Using unfoldings to avoid the state explosion problem in the verification of asynchronous circuits. In Computer Aided Verification (CAV), volume 663 of LNCS, pages 164-177. Springer Berlin Heidelberg, 1992.

[McM95] Kenneth L. McMillan. A technique of state space search based on unfolding. Formal Methods in System Design (FMSD), 6(1):45-65, 1995.

[MR97] Stephan Melzer and Stefan Römer. Deadlock checking using net unfoldings. In Computer Aided Verification (CAV), volume 1254 of $L N C S$, pages 352-363. Springer Berlin Heidelberg, 1997.

[Mur89] Tadao Murata. Petri nets: Properties, analysis and applications. Proceedings of the IEEE, 77(4):541-580, 1989.

[NPW81] Mogens Nielsen, Gordon D. Plotkin, and Glynn Winskel. Petri nets, event structures and domains, Part I. Theoretical Computer Science (TCS), 13:85-108, 1981.

[Pet77] Carl A. Petri. Non-Sequential Processes. Translation of a Lecture given at the IMMD Jubilee Colloquium on "Parallelism in Computer Science", Unversität Erlangen-Nürnberg. Translated by Philip Krause and John Low., Petri, C.A., St. Augustin: Gesellschaft für Mathematik und Datenverarbeitung Bonn, Interner Bericht ISF-77-5, 1977 . 
[PLtH14] Artem Polyvyanyy, Marcello La Rosa, and Arthur H.M. ter Hofstede. Indexing and efficient instance-based retrieval of process models using untanglings. In Advanced Information Systems Engineering (CAiSE), volume 8484 of LNCS, pages 439-456. Springer International Publishing, 2014.

[Pnu77] Amir Pnueli. The temporal logic of programs. In Annual Symposium on Foundations of Computer Science (FOCS), pages 46-57. IEEE Computer Society, 1977.

[PW13] Artem Polyvyanyy and Matthias Weidlich. Towards a compendium of process technologies: The jBPT library for process model analysis. In CAiSE Forum, volume 998 of CEUR Workshop Proceedings, pages 106-113. CEUR-WS.org, 2013.

$\left[\mathrm{PWC}^{+} 14\right]$ Artem Polyvyanyy, Matthias Weidlich, Raffaele Conforti, Marcello La Rosa, and Arthur H.M. ter Hofstede. The 4C spectrum of fundamental behavioral relations for concurrent systems. In Petri Nets, volume 8489 of $L N C S$, pages 210-232. Springer International Publishing, 2014.

[Rac78] Charles Rackoff. The covering and boundedness problems for vector addition systems. Theoretical Computer Science (TCS), 6(2):223-231, 1978.

[Rei13] Wolfgang Reisig. Understanding Petri Nets: Modeling Techniques, Analysis Methods, Case Studies. Springer, 2013.

[RSK13] César Rodríguez, Stefan Schwoon, and Victor Khomenko. Contextual merged processes. In Petri Nets, volume 7927 of LNCS, pages 29-48. Springer Berlin Heidelberg, 2013.

[SNW96] Vladimiro Sassone, Mogens Nielsen, and Glynn Winskel. Models for concurrency: Towards a classification. Theoretical Computer Science (TCS), 170(1-2):297-348, 1996.

[Tar97] Igor V. Tarasyuk. Equivalence Notions for Models of Concurrent and Distributed Systems. PhD thesis, A.P. Ershov Institute of Informatics Systems, Siberian Division of the Russian Academy of Sciences, Novosibirsk, Russia, December 1997.

[vdA97] Wil M.P. van der Aalst. Verification of workflow nets. In Petri Nets, volume 1248 of LNCS, pages $407-426$. Springer Berlin Heidelberg, 1997.

[vGV87] Rob J. van Glabbeek and Frits W. Vaandrager. Petri net models for algebraic theories of concurrency. In Parallel Architectures and Languages Europe (PARLE), volume 259 of LNCS, pages 224-242. Springer Berlin Heidelberg, 1987. 